\title{
Electromigration and the structure of metallic nanocontacts
}

\author{
R. Hoffmann-Vogel ${ }^{\text {a) }}$ \\ Physikalisches Institut and Institut für Angewandte Physik, Karlsruhe Institute of Technology, \\ D-76131 Karlsruhe, Germany
}

(Received 9 March 2017; accepted 3 May 2017; published online 7 August 2017)

This article reviews efforts to structurally characterize metallic nanocontacts. While the electronic characterization of such junctions is relatively straight forward, usually it is technically challenging to study the nanocontact's structure at small length scales. However, knowing that the structure is the basis for understanding the electronic properties of the nanocontact, for example, it is necessary to explain the electronic properties by calculations based on structural models. Besides using a gate electrode, controlling the structure is an important way of understanding how the electronic transport properties can be influenced. A key to make structural information directly accessible is to choose a fabrication method that is adapted to the structural characterization method. Special emphasis is given to transmission electron microscopy fabrication and to thermally assisted electromigration methods due to their potential for obtaining information on both electrodes of the forming nanocontact. Controlled electromigration aims at studying the contact at constant temperature of the contact during electromigration compared to studies at constant temperature of the environment as done previously. We review efforts to calculate electromigration forces. We describe how hot spots are formed during electromigration. We summarize implications for the structure obtained from studies of the ballistic transport regime, tunneling, and Coulomb-blockade. We review the structure of the nanocontacts known from direct structural characterization. Singlecrystalline wires allow suppressing grain boundary electromigration. In thin films, the substrate plays an important role in influencing the defect and temperature distribution. Hot-spot formation and recrystallization are observed. We add information on the local temperature and current density and on alloys important for microelectronic interconnects. Published by AIP Publishing.

[http://dx.doi.org/10.1063/1.4994691]

\section{TABLE OF CONTENTS}

I. INTRODUCTION

II. METHODS FOR THE FABRICATION OF

NANOCONTACTS $\ldots \ldots \ldots \ldots \ldots \ldots \ldots \ldots$

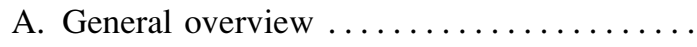

B. Mask evaporation ................

C. STM-based nanoindentation and contacting of planar structures . . . . . . . . . . . .

D. TEM fabrication $\ldots \ldots \ldots \ldots \ldots \ldots \ldots$

III. ELECTROMIGRATION THINNING . . . . . . .

A. Electromigration in disordered metals ......

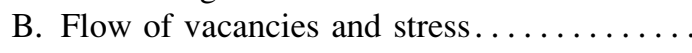

1. Flux divergences ...............

2. Relation of electromigration and local stress-Blech length . . . . . . . . . . .

C. The binding energies $\ldots \ldots \ldots \ldots \ldots \ldots$

D. The direct force and the wind force.......

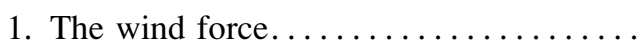

2. The direct force $\ldots \ldots \ldots \ldots \ldots \ldots \ldots$

2

3

3

3

4

5

5

5

7

7

8

8

9

9

10

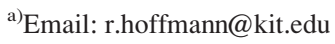

3. Combined approaches.............

E. Using electromigration to generate metallic nanocontacts ................ 11

1. Controlled electromigration ......... 12

2. Temperature estimates ........... 13

3. Time-scale of controlled electromigration ...............

IV. ELECTRONIC CHARACTERIZATION METALLIC NANOCONTACTSIMPLICATIONS FOR THE STRUCTURE .... 13

A. Macroscopic part, thermal regime ....... 13

1. Initial stages of current flow through freshly prepared samples .......... 13

2. Size of the heated region .......... 14

3. Several weak spots in one metallic

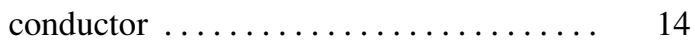

B. Ballistic regime ............... 15

1. Overview................. 15

2. Histograms generated from electromigration studies .......... 16

3. Self-breaking as observed in the

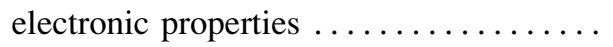


4. The structure of nanoscale metallic junctions in the ballistic regime .......

C. Electron transport across a gap..........

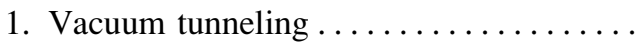

2. Transition to field emission ..........

3. Coulomb blockade...............

D. Reversing the current direction-cyclic

behavior .....................

E. Role of the chemical environment .......

1. Diffusive regime $\ldots \ldots \ldots \ldots \ldots \ldots$

2. Few atom contacts.............

3. Consequences for studies of nanoscale metallic junctions. .............

V. DIRECT STRUCTURAL

CHARACTERIZATION OF METALLIC

NANOCONTACTS . . ..............

A. Imaging methods and their limitations concerning imaging of nanocontacts ......

B. Elementary processes..$\ldots \ldots \ldots \ldots \ldots$.

1. Atomic hops and surface diffusion.....

2. Surface electromigration............

C. Single-crystalline nanowires $\ldots \ldots \ldots \ldots \ldots$

D. Grain boundary electromigration ........

E. Substrate-supported thin films..........

1. Fabrication details of thin-film wires with a constriction.............

2. Overall thinning process...........

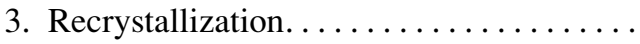

4. Hot-spot and notch formation .........

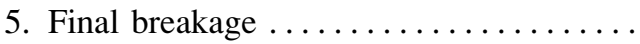

F. Free-standing thin films $\ldots \ldots \ldots \ldots \ldots \ldots$

VI. LOCAL PHYSICAL QUANTITIES-

TEMPERATURE, CURRENT DENSITY, AND

SURFACE POTENTIAL..............

A. Local temperature distribution .........

B. Local current density and potential near

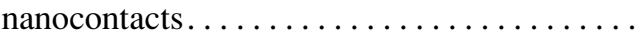

VII. NANOCONTACTS OF ALLOYS . .........

A. Electromigration of alloys ...........

B. Structural evolution during electromigration

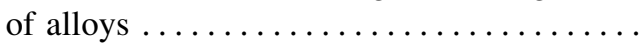
VIII. CONCLUSIONS $\ldots \ldots \ldots \ldots \ldots \ldots \ldots \ldots$

\section{INTRODUCTION}

Metallic junctions are important in microelectronics as integrated-circuit interconnects. As the functional elements in such integrated circuits become smaller, the metallic interconnects are reduced in size at the same rate. One important issue has been electromigration failure (Ho and Kwok, 1989 and Pierce and Brusius, 1997). In the 1990s, the main material used for interconnects in microelectronic circuits was changed from $\mathrm{Al}$ to $\mathrm{Cu}$. $\mathrm{Cu}$ is more resistant to electromigration because it has a higher melting temperature. This is equivalent to stronger $\mathrm{Cu}-\mathrm{Cu}$ chemical bonds and in consequence larger energy barriers for diffusion and electromigration. Today, electromigration is still an important point for reliability (Oates, 2015). Already today, parts of local interconnects are from $\mathrm{W}$ with even higher melting temperature for improved electromigration resistance and further steps such as replacing $\mathrm{Cu}$ by $\mathrm{W}$ in thin film interconnects (lines) are discussed (Beyne et al., 2016). For small interconnects, the structural arrangement at the atomic scale, for example, the presence or absence of grain boundaries or atomic surface steps, becomes increasingly important. Cyclic electromigration behavior at the atomic scale has been observed (Schirm et al., 2013). It has been proposed that new memory devices could be built on the basis of atomic switches (Aono and Hasegawa, 2010 and Suga et al., 2016) and first systems are being commercialized (Kaeriyama et al., 2005).

When reducing the size of an interconnect, a new electronic transport regime is reached when the interconnect's size becomes comparable to the metal's inelastic mean free path. In this regime, the influence of scattering reduces and the quantum properties of the electrons become even more important compared to a bulk metal. Immediately after the observation of plateaus in the electronic conductance through nanoscale metallic junctions measured as a function of junction size (Muller et al., 1992), the question was raised whether these plateaus arise from quantum-mechanical quantization of electronic transport via conduction channels (electronic waveguide modes), as demonstrated earlier for semiconductor channels (van Wees et al., 1988 and Wharam et $a l ., 1988$ ) or from the atomic structure of the junction. In many studies, the close relation of both aspects has become evident [see Agrait et al. (2003) for an overview]: On the one hand, the structure of nanoscale metallic junctions is governed by quantum effects. For example, closed shell configurations of the atoms, the electrons, and the influence of confined electronic states on the structure have been clearly observed (Yanson et al., 1999 and Stepanyuk et al., 2004). On the other hand, the details of the atomic structure of the contact determine quantum transport, i.e., ballistic transport with a strong influence of the wave nature of electrons, usually observed at low temperatures.

In addition, full control of the structural properties and chemical composition of metallic contacts is of utmost importance for molecular electronics studies (Cuevas and Scheer, 2010; Osorio et al., 2008; and Perrin et al., 2015). The conductance of the junction depends strongly on the chemical potential of the molecule which depends on the coupling to the metal and on the coupling to the environment (Moth-Poulsen and Bjørnholm, 2009). The bonding geometry of the leads to the molecule, see Fig. 1, is vital for the conductance of a nanoscale molecule-metal junction (Aradhya and Venkataraman, 2013 and Venkataraman et al., 2006). This bonding geometry depends on the atomic structure of the metallic contact. In fact, when the conductance of such a molecule-metal junction is measured, in histograms of the measured conductance often a big unspecific background is obtained in addition to the signature of the molecule. This background could depend on the chemical environment of the molecule. Coulomb blockade and the Kondo effect have been observed in such molecule-metal junctions (Park et al., 2002; Kubatkin et al., 2003; and Liang et al., 2002) but also in pure metallic nanocontacts (Houck et al., 2005). Structural characterization helps to determine which aspects of the 


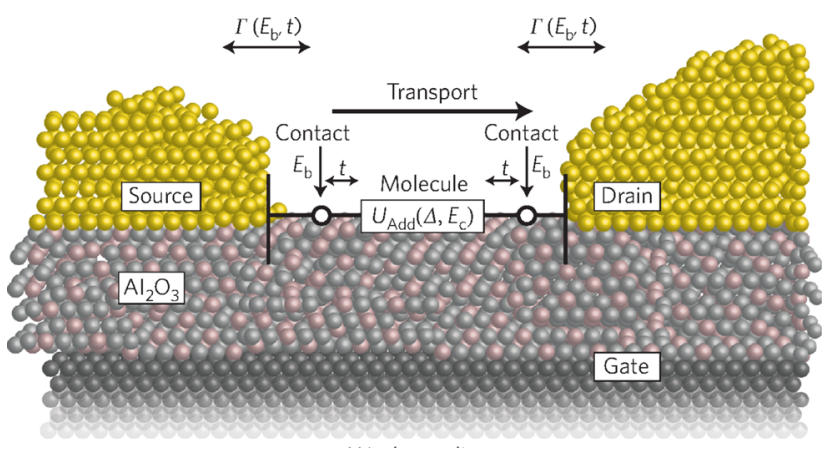

FIG. 1. Artist's view of a molecular junction where the molecule and its contact area with electrodes and substrate are not drawn explicitly. Reprinted with permission from K. Moth-Poulsen and T. Bjørnholm, Nat. Nanotechnol. 4, 552 (2009). Copyright 2009 Macmillan Publishers Limited.

metal-molecule-substrate interaction are responsible for these effects.

Structural characterization of nanoscale metallic junctions is technically challenging. The main methods used to study electronic transport of nanoscale metallic junctions, such as the mechanically controlled break-junction technique, are not adapted for imaging purposes, in particular for high-resolution imaging.

In this review, we discuss electromigration of metallic nanocontacts and the structural properties of nanocontacts. We first discuss fabrication methods of nanoscale metallic junctions. A special emphasis is given on transmission electron microscopy (TEM)-based fabrication methods and on electromigration methods, because these have allowed high-resolution structural characterization. We review how controlled electromigration aims at keeping the temperature of the contact constant during electromigration. The strong temperature dependence that had led previously to thermal runaway is lifted, and the contact can be studied at experimentally accessible time scales. We give a summary of efforts to calculate electromigration forces. We then briefly review the electronic properties of nanocontacts with special emphasis on the implications for the contact's structure. We describe how hot-spot formation manifests itself in $\mathrm{I}(\mathrm{V})$ measurements during controlled electromigration thinning. We discuss the ballistic regime as well as the tunneling regime and observations of Coulomb blockade. Then, we turn to the results of direct structural characterization. Single-crystalline nanowires are fabricated using elaborate growth techniques. In these, grain boundary electromigration is suppressed. We discuss how recrystallization and hot-spot formation alter the structure of substrate-supported thin films. We devote a section on the local temperature and current density obtained from special scanning probe microscopy measurements. We describe recent results on alloys since these are important for electromigration in microelectronics interconnects.

Experiments in liquids and magnetic effects are only discussed occasionally. We do consider gaseous impurities, but we do not focus on molecular electronics although the nanocontacts discussed here are in many cases well-suited to contact molecules. In addition, we refer the reader to previous reviews of electromigration (Ho and Kwok, 1989; Pierce and Brusius, 1997; Lloyd, 1997; Sorbello, 1998; de Orio et al., 2010; and Ceric and Selberherr, 2011), the electronic properties of nanoscale metallic junctions (Agraït et al., 2003), and the physical and electronic structure of atomic scale nanowires from a theoretical point of view (Bowler, 2004 and Pecchia and Carlo, 2004).

\section{METHODS FOR THE FABRICATION OF NANOCONTACTS}

\section{A. General overview}

It is desirable that the size of a metallic nanocontact should be controllable down to atomic dimensions, because in metals the Fermi wavelength is on the order of the size of an atom. Finally, nanogap disconnected electrodes are needed for molecular electronics purposes (Li et al., 2010 and Song et al., 2011). A technique often used to obtain atomic-scale contacts is the mechanically controlled breakjunction method, where a free-standing metallic bridge is fabricated by means of electron beam lithography on a flexible substrate. By bending the substrate in the center while keeping the sides fixed, the metallic bridge is elongated and thinned mechanically at the same time. This is usually done under cryogenic vacuum conditions to keep the metallic conductor clean (Muller et al., 1992 and van Ruitenbeek et al., 1996). While the technique is very successful for studying the electronic properties, the geometry of the bridge usually makes it difficult to image the nanocontact itself. In general, the nanocontact is located inside a relatively thick (about several 100-1000 nm) metallic bridge needed for mechanical stability and chemical robustness. This large film thickness makes it difficult to access the nanocontact itself.

Other methods to fabricate nanoscale metallic junctions are available. To allow for successful structural characterization, the fabrication method of the nanocontacts should be adapted to the characterization method to be used, and we will focus here on such fabrication methods. Mainly two types of characterization methods come into mind, electron microscopy-based methods and scanning probe methods. For electron microscopy (i.e., SEM, TEM, or related techniques), planar, free-standing nanocontacts or nanocontacts supported by an electron-transparent substrate are needed (Kondo and Takayanagi, 1997). For scanning probe techniques, two different geometries have been used: planar nanocontacts on insulating substrates have been imaged by scanning force microscopy (SFM) (Stöffler et al., 2012; Girod et al., 2012; Durkan and Welland, 2000a; and Durkan et al., 1999), or the tip of a scanning tunneling or force microscope has been brought into contact with a metallic surface (Gimzewski and Möller, 1987; Limot et al., 2005; Ternes et al., 2011; Oliver et al., 2012; and Hansen et al., 2000). The metallic contacts are required to be atomically flat. These requirements and methods will be discussed in more detail in the following.

\section{B. Mask evaporation}

Many fabrication techniques for small structures are based on evaporation through a mask. Such techniques are often used for making metallic bridges suitable for 
electromigration thinning. For mask evaporation techniques, there are mainly two challenges, one is to fabricate small structures in the mask and the other is to bring the mask as close as possible to the substrate. Fabricating structures in masks is often much easier than producing similar structures on surfaces, because the choice of material for the mask is naturally much wider. In particular, $\mathrm{Si}_{3+x} \mathrm{~N}_{4-x}$, PMMA, and photoresist are often suitable materials. PMMA and photoresist also offer the advantage that the mask is very close to the surface, because it is deposited often by spin-coating directly onto the surface of the substrate. In this way, even exceptionally small nanogaps of size below $1 \mathrm{~nm}$ have been produced (Fischbein and Drndic, 2006). If structures on a large scale are to be used, a statistical alignment technique will help produce at least one small gap over the full space used (Steinmann and Weaver, 2005). Subwavelength nanostructures were fabricated from optical lithography using diffraction (Jung et al., 2012).

Besides being an advantage, positioning the mask directly on the sample also constitutes an important difficulty, because after making the nanostructures, PMMA must be removed by solvents, which often leaves residues on the sample surface (Stöffler et al., 2011). Another approach is to use a free-standing metallic contact fabricated on top of a slit to prevent contamination (Anaya et al., 2003). Resistless fabrication of nanocontacts has been reviewed (Vazquez-Mena et al., 2015). Here, we particularly mention stencil masks (Zahl et al., 2005) that can be approached to the surface and removed mechanically after creation of the nanostructure. Some approaches use static stencils of $\mathrm{Si}_{3+x} \mathrm{~N}_{4-x}$ membranes (Gärtner et al., 2006), see Fig. 2, Si (Otsuka et al., 2004), or even carbon nanotubes (Lefebvre et al., 2000). The technique has been developed further, by reinforcing the $\mathrm{Si}_{3+x} \mathrm{~N}_{4-x}$ membranes used as a stencil to make them more robust to the stress they experience after deposition of a metallic layer on top (Fostner et al., 2010).

A more advanced approach is to combine a mask with a nanopositioning system to form a dynamic stencil (Lüthi et al., 1999). The main idea is to reuse the mask at several

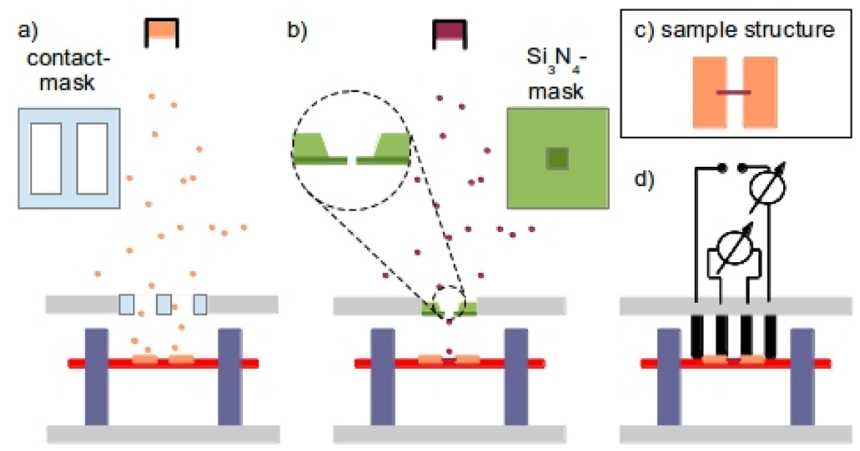

FIG. 2. Example of a mask evaporation process: (a) First a mask for making large contacts is placed in front of the sample. (b) This mask is then removed and $\mathrm{Si}_{3+x} \mathrm{~N}_{4-x}$ membrane is placed in a similar position to fabricate small metallic structures. (c) This mask is also removed and (d) the sample is contacted by macroscopic leads to perform $\mathrm{I}(\mathrm{U})$ measurements and thinning schemes for producing a nanoscale metallic junction from the metallic bridge fabricated in this way. Reprinted with permission from D. Stöffler and R. Hoffmann-Vogel, J. Vac. Sci. Technol. B 33, 013201 (2015). Copyright 2015 American Vacuum Society. positions and to write patterns by moving the sample and the mask with respect to each other while evaporating the metal. The nanopositioning device is in many ways similar to a STM scanner, see Fig. 3. The technique can be used to fabricate structures previously impossible to make and is compatible with fundamental surface science methods such as heating and sputter cleaning (Egger et al., 2005). The accuracy of positioning the nanopattern is better than $100 \mathrm{~nm}$ (Guo et al., 2007). For a translation of $900 \mu \mathrm{m}$, an accuracy of $2.5 \mu \mathrm{m}$ has been achieved (Savu et al., 2008). The technique is cost-efficient and can be used to pattern even areas of $\mathrm{cm}^{2}$ size (Blech et al., 2006).

\section{STM-based nanoindentation and contacting of planar structures}

In a scanning tunneling microscope, a metallic tip is moved with great precision with respect to a conductive sample (Binnig et al., 1982). In general, a contact between the tip and the sample is avoided when the tip is brought into close vicinity of the sample such that electronic tunneling occurs after applying a voltage (Binnig and Rohrer, 1982). The tunneling current is strongly distance dependent and is usually used to control the tip-sample distance in a feed-back loop. By spectroscopic means, the contribution of surface nanostructures can be separated from the total current and information about the local electronic structure can be gained (Tromp, 1989 and Schneider, 2002). In this way, it is even possible to separate the contribution of a metallic structure from a metallic substrate, e.g., a monoatomic metallic chain on a metal surface (Nilius et al., 2002; 2003; and Meyer et al., 2002).

The main method to study metallic nanocontacts with STM is to use the ability of the STM to precisely position the tip to indent the tip carefully into the surface and in this way create a metallic nanocontact (Gimzewski and Möller,

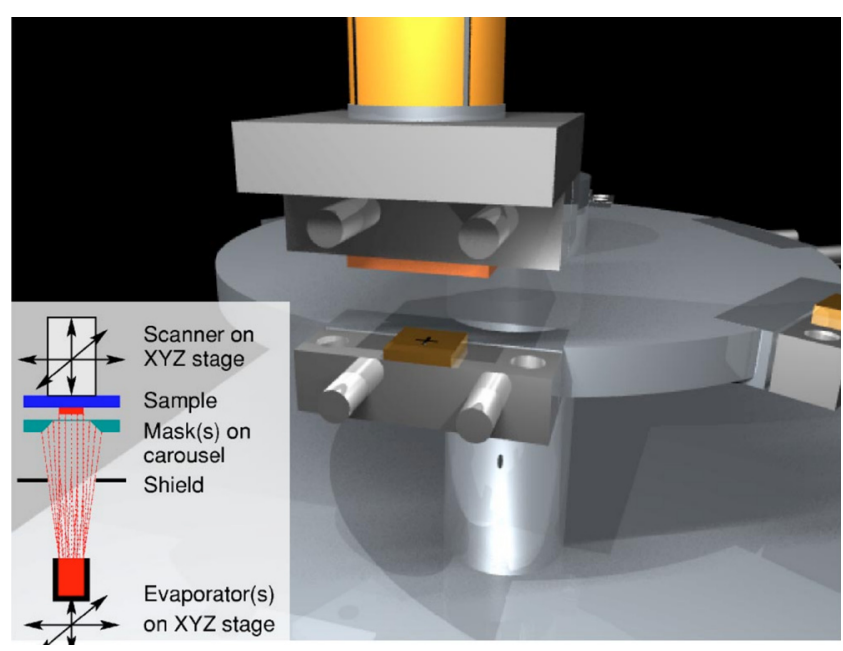

FIG. 3. Mask located in a carousel that can be turned to move different masks in front of the sample. The sample is mounted on a piezoelectric scanner such that the mask can be dynamically applied to different positions on the sample. Inset: An evaporator located under the carousel allows the evaporation of a material to the sample surface via the mask. Reproduced with permission from Rev. Sci. Instrum. 76, 023707 (2005). Copyright 2015 AIP Publishing. 

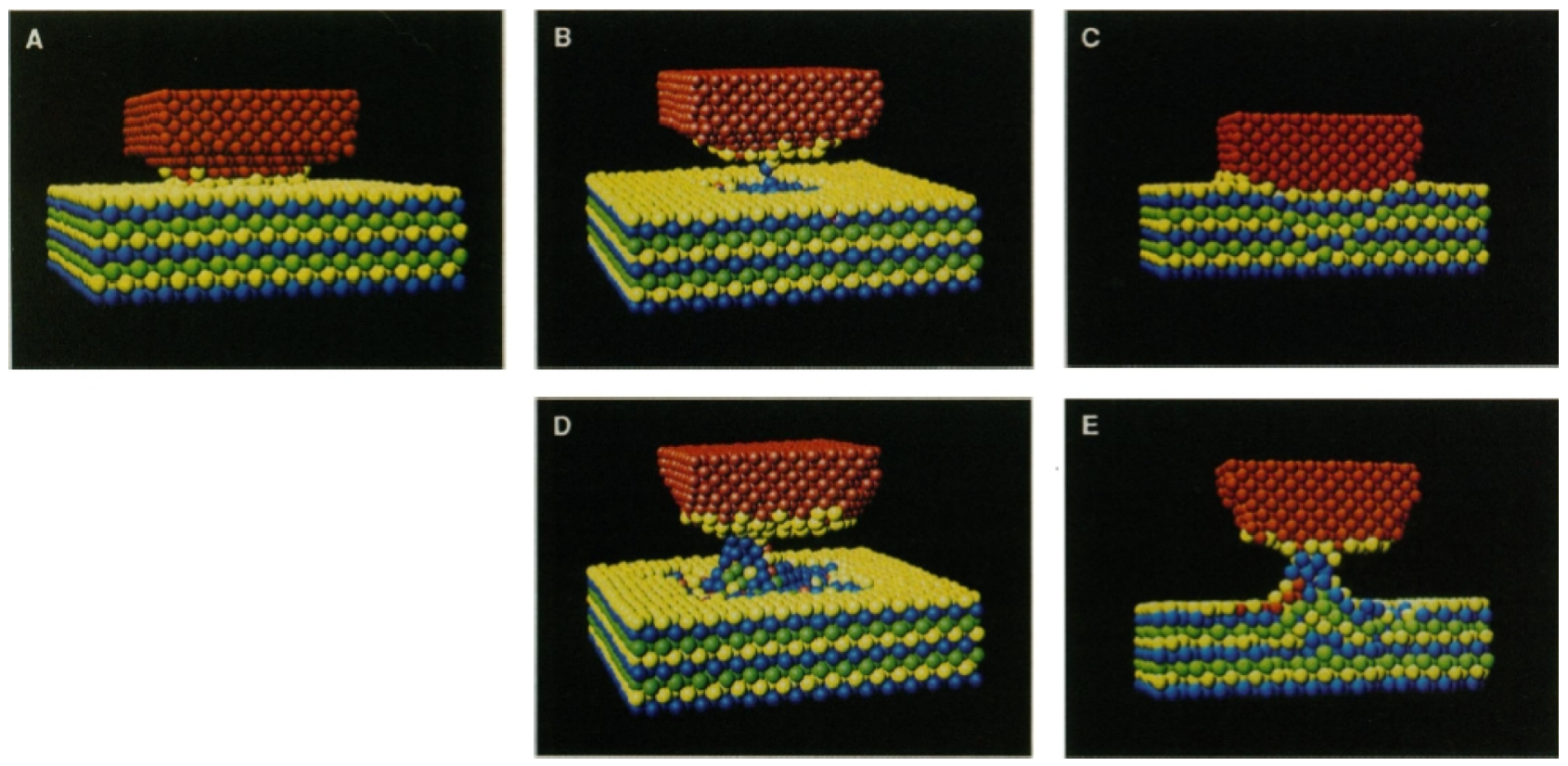

FIG. 4. Molecular dynamics simulations of the approach of a Ni tip to a Au surface. (a) The situation after jump to contact. (b) The tip is retracted again and an atomically thin connection is formed. (c) A cut through the system at the point of maximum indentation. (d) After retraction, there has been partial intermixing of tip and substrate atoms and the metallic connection remains. (e) A cut through the system after retraction corresponding to the same distance as image (d). Reprinted with permission from Landman et al., Science 248, 454 (1990). Copyright 1990 American Association for the Advancement of Science (Landman et al., 1990).

1987; Limot et al., 2005; Ternes et al., 2011; Zhang et al., 2011; and Oliver et al., 2012). In Fig. 4(a), simulation of such a process is shown. An advantage of the method is that the area on the surface used for indentation can be carefully chosen. A disadvantage of this method is that only one of the two electrodes, the surface, can be imaged. The tip could be imaged by field ion microscopy (Lucier et al., 2005). Information about the contact itself is obtained through interpretation of the obtained electronic information (Oliver et al., 2012). If SFM is used, the force jumps due to tipsample atomistic rearrangements can be measured in addition to the electric current and it is possible to relate both measured quantities (Paul et al., 2014).

As a second way of how STM could be used for studying metallic nanocontacts, it is sometimes proposed to study planar nanocontacts supported by an insulating surface using STM (Stöffler et al., 2011; 2012). A conductive or even metallic substrate is covered by a thin insulating layer. Tunneling should be possible if the nanocontacts are metallic and electrically connected or if the substrate is thin enough to tunnel through the insulating layer (Schintke and Schneider, 2004).

A further development of the technique is to use four STM tips and to contact a structure on the surface using these four tips (Homoth et al., 2009; Kaspers et al., 2009; Okino et al., 2005; and Lin et al., 2004). The main challenge here is to avoid thermal oscillations of the relatively long and thin metallic tips. This geometry additionally offers the possibility of manipulating atoms or molecules in the junction (Samitsu et al., 2005) and of using one of the tips as a gate (Bietsch and Michel, 2002). The gate efficiency for this configuration has been investigated theoretically (Damle et al., 2002). The STM tip gate is movable in contrast to break-junctions with an added gate electrode (Martin et al., 2010).

\section{TEM fabrication}

Since the 1990s, high-current TEM bombardment has been successfully used for nanocontact fabrication and the results are among the most successful high-resolution studies of nanoscale metallic junctions. To make nanocontacts by TEM bombardment, a 3-5 nm thin self-supported Au film is deposited on a carbon grid. Nanoholes are created by focusing the electron beam and thus achieving a local electron bombardment with a high current density (100-120 A/ $\mathrm{cm}^{2}$ ) (Kondo and Takayanagi, 1997 and Coura et al., 2004). The remaining Au film areas between the holes ideally have the form of nanowires. The current density is subsequently reduced to allow imaging $\left(30 \mathrm{~A} / \mathrm{cm}^{2}\right)$. During imaging, the nanowires spontaneously evolve in shape (Kondo and Takayanagi, 1997). The nanowires obtained in this way show a remarkable stability and interesting structures as will be discussed in Sec. V.

Another variant is to combine the TEM approach with the STM nanoindentation approach and build a TEM that can accommodate a STM in the space of its imaging plane (Ohnishi et al., 1998), see also Fig. 5. The electronic properties can then be investigated in parallel (Oshima and Kurui, 2013). TEM can also be used to deposit the material from the gas phase. In the simplest case, this material has a large carbon content, but it could also be metallized, e.g., by electromigration (Han et al., 2012).

\section{ELECTROMIGRATION THINNING}

\section{A. Electromigration in disordered metals}

Electromigration has been studied intensely for the past 45 years mainly because of its role as a failure mechanism in electronic circuits (Blech and Meieran, 1967; Ho and Kwok, 


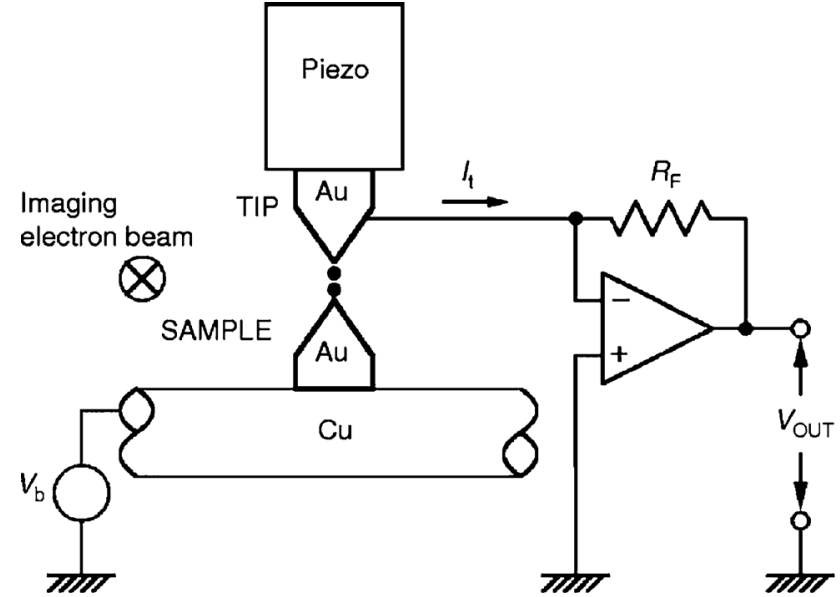

FIG. 5. STM set-up used in the literature for studying nanoscale metallic junctions with TEM. The set-up very much resembles a standard STM setup, except that this instrument is used under the electron beam of a TEM. Reprinted with permission from Ohnishi et al., Nature 395, 780 (1998). Copyright 1998 Macmillan Publishers Limited.

1989; Pierce and Brusius, 1997; Lloyd, 1997; and HauRiege, 2004). Even for small current densities, a prolonged use at near-room-temperature operating conditions can lead to failure of electronic circuits. Starting from the 1990s (Ralls et al., 1989 and Park et al., 1999), the idea has been developed to use electromigration as a method for making metallic nanocontacts to molecules. An advantage compared to other techniques is that as a matter of principle, many contacts can be made in parallel with this technique [up to 16 in. (Johnston et al., 2007)] and that it is very reliable because a big portion of the gaps fabricated are smaller than $10 \mathrm{~nm}$ in size with 9 out of 16 of $1-2 \mathrm{~nm}$ distance in one study (Hadeed and Durkan, 2007). In order to apply the technique, thermal runaway must be avoided which can lead to melting of the structures. As another advantage for electromigrated nanocontacts, compared to mechanically controlled breakjunctions, the thickness of the metallic film at the origin of the thinning process is often below $100 \mathrm{~nm}$ and could in principle be reduced further to atomic dimensions.

In a current-carrying metallic lead, apart from the electrostatic force, also called direct force, the momentum transfer from the flow of electrons causes another contribution to the force on an ion or defect. This contribution is called the wind force and arises from electronic scattering mechanisms. Both forces are proportional to the local electrostatic field at the position of the atom considered and can be summarized in the following equation:

$$
F=\left(Z_{1}-Z_{2}\right) e E,
$$

where $Z_{1}$ and $Z_{2}$ are proportionality constants describing the effective charge numbers the electrostatic field is acting on. $Z_{1}$ for the wind force differs for grain boundary, surface, and bulk electromigration [for numerical values see, for example, Doi et al. (2003)]. $Z_{2}$ for the direct force includes screening of the local charge. In the case of aluminum, gold, or copper, both forces oppose each other (i.e., the screened metallic ions appear to have a positive charge $Z_{2}$ ) and the influence of the wind force is stronger such that the atoms are pushed in

the direction of the electron flow. However, in particular for impurity atoms in a metallic matrix, the screened atoms may show an overall negative effective charge $Z_{2}$ (Ho and Kwok, 1989 and Dekker et al. 1997). One of the main difficulties of electromigration studies is to determine the precise value of these charges.

While metallic ions usually appear to have a positive charge, vacancies move in the opposite direction. Since most metals contain vacancies (Kirkendall et al., 1939), it is conceptually much simpler to think of electromigration forces causing a vacancy flux

$$
J_{v}=D \frac{c}{k T}\left(Z_{1}-Z_{2}\right) e E,
$$

where $c$ is the vacancy concentration and $D$ is the diffusivity (Blech and Herring, 1976; Pierce and Brusius, 1997; HauRiege, 2004; and de Orio et al., 2010).

In order to understand the role of electromigration forces and of thermally activated diffusion, sometimes also called thermomigration, let us assume for simplicity that the resulting force from Eq. (1) was constant as a function of position for a particular atom. A surface atom then-together with the oscillating potential of its environment-resides in a tilted washboard-potential, see Fig. 6(a). Thermally activated diffusion allows the atom to hop from one minimum to another with the rate

$$
\gamma=\gamma_{0} \exp \left\{-E_{A} /\left(k_{B} T\right)\right\},
$$

where $\gamma_{0}$ is the attempt frequency, $E_{A}=\varepsilon_{B}-\varepsilon_{1}$ is the hopping energy barrier, and $\varepsilon_{B}$ and $\varepsilon_{1}$ are the maximal energy between the two states and the energy in the original state, as defined in Fig. 6(b). Thus, there is a directional diffusion of atoms for electromigration. For thermomigration or simple diffusion, the potential would be assumed to consist of a number of degenerate minima without tilt. Such a degenerate potential would lead to equal diffusion in all degenerate crystallographic directions. In reality, the situation is much more complicated, because not only the binding energies differ for atoms located at bulk, surface, step edge, grain boundary, or defect positions, etc., but also the electromigration forces and the details of the hopping process depend on the diffusion mechanism as known from surface diffusion studies.

Electromigration failure is the consequence of a discontinuity in the mass flow along a wire. Discontinuities in the

a)
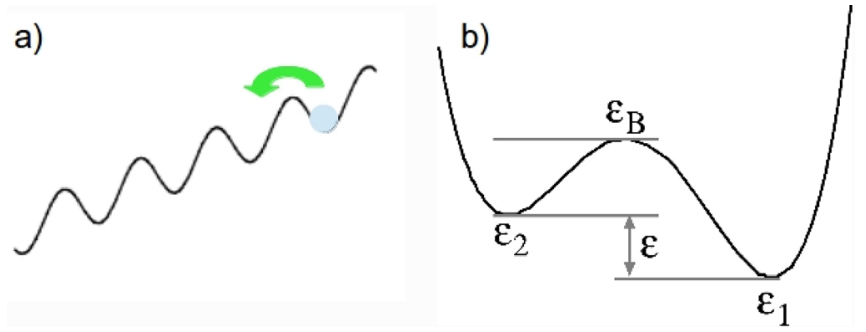

FIG. 6. (a) If microscopic and macroscopic effects are to be summarized in one picture, diffusion under the influence of electromigration forces can be understood as hopping in a tilted washboard-potential. (b) Energy landscape for a hopping atom with a definition of its energy parameters. 
mass transport lead to an excess mass in the material or to void formation, and thus, stress builds up as discussed below. During electromigration failure, the resistivity at one particular point in the metal becomes larger than that of its environment, which in turn is the reason for a higher temperature at this emerging hot-spot. The higher temperature again accelerates diffusion and electromigration in this area and increases the local resistivity further. This self-accelerating process mainly causes electromigration failure of metallic leads. In the extreme case, it can lead to melting of the contact.

Usually, the median time to failure $t_{50}$ is studied at constant temperature of the environment and voltage, because this is of technological interest. It can be described using the Black equation (Black, 1967)

$$
t_{50}=\frac{A}{J^{2}} e^{-E_{A} / k_{B} T},
$$

where $J$ is the current density and $A$ is a material-dependent constant. The diffusivity and electromigration failure are both strongly temperature dependent, thermally activated quantities. Shatzkes and Lloyd specified that the coefficient $A$ depends on temperature as $A=B T^{2}$ (Shatzkes and Lloyd, 1986). Lloyd specified that the median time to failure combines the time for void nucleation and the time for electromigration failure (Lloyd, 1991).

\section{B. Flow of vacancies and stress}

\section{Flux divergences}

The flow of vacancies causes mechanical stress to build up in the wire. The stress usually retards electromigration. Therefore, a back-diffusion term

$$
J_{b}=-D \frac{\partial c}{\partial x}
$$

is included and one obtains the total flux

$$
J=-D \frac{\partial c}{\partial x}+D \frac{c}{k T}\left(Z_{1}-Z_{2}\right) e E .
$$

This flux is in equilibrium as long as the number of incoming atoms in a given volume equals the number of outflowing atoms. If this dynamic equilibrium is disturbed, locally the number of atoms or vacancies can mathematically diverge or approach zero. Such flux divergences cause extrusions or voids to form both of which can lead to failure.

Flux divergences can be generated, for example, by gradients in grain size, gradients in the dimension of the wire, or temperature gradients (Pierce and Brusius, 1997). Such gradients commonly occur at the electrodes where the interconnect is contacted, because at these positions usually the dimension of the wire changes. Another cause for flux divergences is triple connections of grain boundaries (see Fig. 7), because the flux of incoming atoms for a general orientation of the grain boundaries is not equal to the flux of outgoing atoms at these positions. Since grain-boundary-
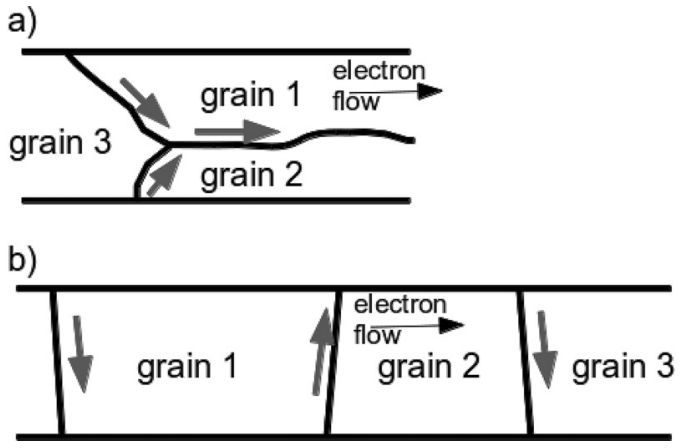

FIG. 7. (a) Triple point where three grain boundaries meet. At this point, more material is added that can be removed and compressive stress builds up. (b) This does not happen in a bamboo-like wire, because per definition it does not have triple points.

electromigration is dominant in many cases, these are of great importance. Samples without such triple connections are called bamboo structures. As one can see in Fig. 8, indeed, it is found experimentally that the median time to failure depends on the size of the sample with respect to the grain size. As expected, the median time to failure increases significantly when one changes to a bamboo structure (Cho and Thompson, 1989). Also, calculations using a phasefield-crystal model show how voids grow at triple connections of grain boundaries, Fig. 9 (Wang et al., 2016).

To inhibit electromigration failure, it is important to create an unfavorable situation for extrusion formation. Covering by additional layers can counter-balance the stress, inhibit extrusion formation, and thus suppress electromigration (Hau-Riege, 2004). In addition, the overlayer can shunt the current and therefore void nucleation does not immediately lead to failure (Castro et al., 2007).

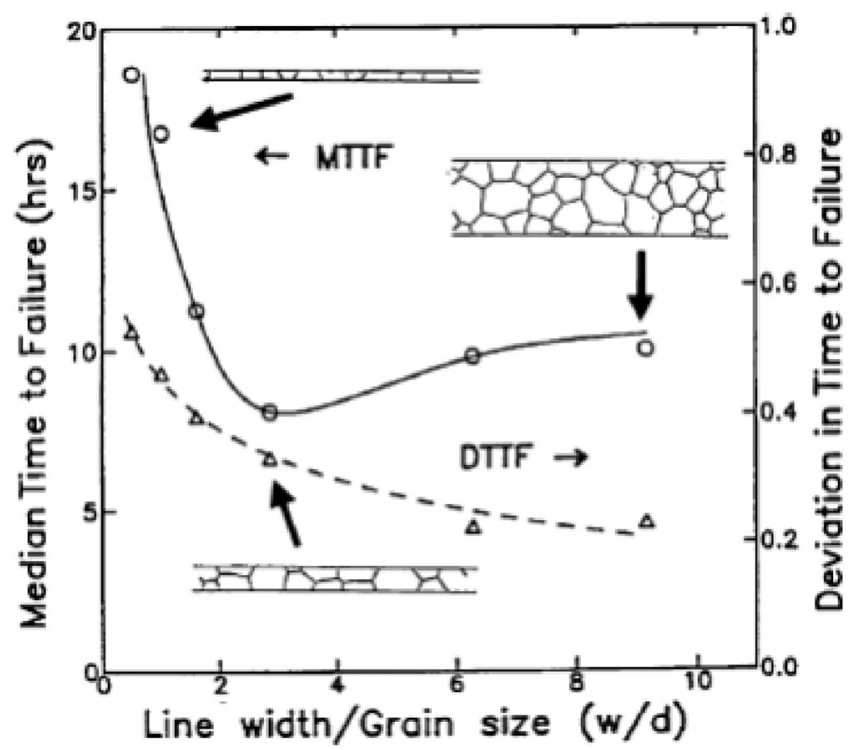

FIG. 8. Measured median time to failure as a function of sample size with respect to grain size. Reproduced with permission from Appl. Phys. Lett. 54, 2577 (1989). Copyright 1989 AIP Publishing. 


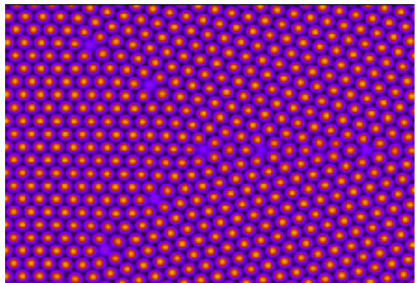

(a)

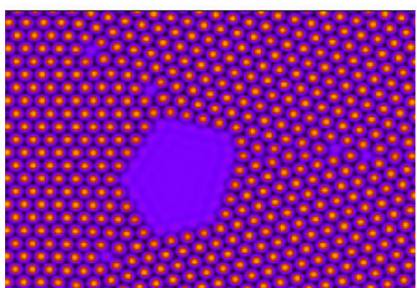

(c)

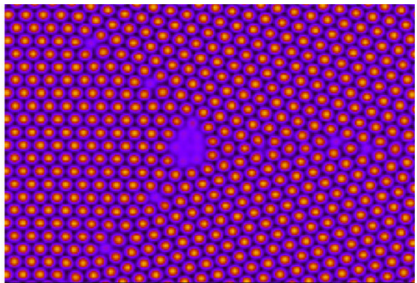

(b)

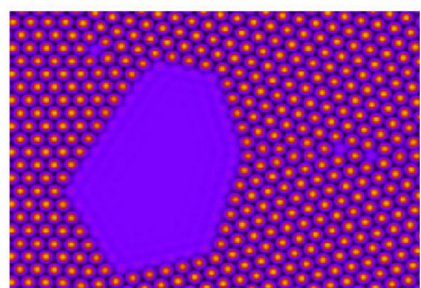

(d)
FIG. 9. Calculated atomic density evolution as a function of time after applying an electric field. Reprinted with permission from Wang et al., Phys. Rev. Lett. 117, 155901 (2016). Copyright 2016 American Physical Society.

\section{Relation of electromigration and local stress-Blech length}

Korhonen derived a relation between the stress built up and the change in atomic concentration $C$ from elastic theory considerations (Korhonen et al., 1993). The change in atoms deposited along the line is related to the dilatational free strains of the material $\varepsilon$, i.e., shear strains are not considered here, such that

$$
\mathrm{d} \varepsilon=\frac{\mathrm{d} C}{C} .
$$

Since this is a continuum theory, the properties must be averaged over a sufficiently large volume. From the three-dimensional elastic theory, the strain can be related to a stress using a matrix $T$ which depends on the elastic properties of the sample mainly given by its Young's modulus $E$, because the coefficients $T_{i, j}$ are related to $E$, the Poisson number $\nu$, and the elastic compliances by relations involving the dimensions of the line and its environment

$$
\varepsilon_{i}=\sum_{j=1}^{3} T_{i, j} \sigma_{j} \quad i=1,2,3 .
$$

From this, Korhonen obtained the relation

$$
\mathrm{d} \varepsilon=\frac{\mathrm{d} C}{C}=\frac{-\mathrm{d} \sigma}{B},
$$

where $B$ is the bulk modulus of the sample that, for isotropic and linear elastic behavior, can be described as $B=E$ / $(3-6 \nu)$. This description together with the continuity equation gives

$$
-\frac{\partial J}{\partial x}=\frac{\partial C}{\partial t}+\frac{C}{B} \frac{\partial \sigma}{\partial t} .
$$

This relation can be used to derive a partial differential equation which describes the stress evolution in the sample

$$
-\frac{\partial \sigma}{\partial t}=\frac{\partial}{\partial x}\left[\frac{D B \Omega}{k T}\left(\frac{\partial \sigma}{\partial x}+\frac{\left(Z_{1}-Z_{2}\right) e E}{\Omega}\right)\right],
$$

where $\Omega$ is the atomic volume. Kirchheim analyzed the temporal evolution of stress built up in the wire (Kirchheim, 1992). Stress effects in different geometries of interconnects and the related stress have been studied and were found to be in agreement with the model (Filippi et al., 2002). Void nucleation due to stress has been studied theoretically using finite-element studies and the nucleation time distribution has been calculated (Dwyer, 2010).

Let us consider a line, a thin film interconnect, where at the end no flux is allowed to occur. The end of the line could be an interconnect passing through the substrate wafer, a via. A steady state occurs when electromigration and backdiffusion are equal.

$$
\left(Z_{1}-Z_{2}\right) e \rho j=\Omega \frac{\partial \sigma}{\partial x},
$$

where $j$ is the current density. This equation yields a linear distribution of the stress along the line

$$
\sigma(x)=\sigma_{0}+\frac{\left|Z_{1}-Z_{2}\right| e \rho j}{\Omega} x,
$$

where $\sigma_{0}$ is the stress at the start of the line, i.e., at $x=0$. If we assume that failure occurs when the stress exceeds a critical value, $\sigma_{c}$, we obtain the condition (Blech, 1976)

$$
j_{1} * L>\left(\sigma_{c}-\sigma_{0}\right) \frac{\Omega}{Z^{*} e \rho},
$$

where $Z^{*}=Z_{1}-Z_{2}$ and $L$ is the length of the line. This means that for a given current density below a certain line length, called Blech length, from this model no failure is expected.

\section{The binding energies}

The binding energies of metallic atoms as well as the activation energy barrier depend strongly on the local environment. Due to the role of thermal activation in electromigration, as it is manifest, e.g., in Black's law, a limited type of spectroscopy of the binding energies can be performed (Blech and Meieran, 1969) through studying electromigration dominated by different diffusion paths. Since thermal activation causes a strong dependence of the frequency of atomic hops on the activation energy barrier, usually there is one dominant process - the one along the reaction path with the lowest activation energy barrier. In this reaction path, there can be several intermediate states with different activation energy barriers, and the process with the largest activation energy barrier will slow down the process and dominate the timescale of hopping.

From general considerations such as the coordination, we expect bulk atoms to have the largest binding energies, and atoms at dislocations, grain boundaries, surfaces, and vacancies to have reduced binding energies. Atoms near defects can have both larger and smaller binding energies compared to the undisturbed solid depending on their 
chemical environment, as an example, see Sen and Aydinol (2008). Due to thermal activation, we expect that the binding energies strongly influence the temperature at which electromigration sets in. This is indeed observed experimentally, see Table I. In addition, single-atom adhesion and binding energies have been studied in nanoscale metallic junctions using the break-junction method (Trouwborst et al., 2008). It is found that these processes are often well described by models assuming a constant elasticity for atomic binding.

The activation energy barriers for different surface diffusion processes have also been calculated by the embedded atom method (Mehl et al., 2000). For $\mathrm{Cu}$ island drift and for void drift, effective atomic diffusion barriers of 0.78-0.79 $\mathrm{eV}$ and $0.73-0.84 \mathrm{eV}$, respectively, were found to be in fair agreement with the experimental values given in the table above.

\section{The direct force and the wind force}

\section{The wind force}

As stated above, usually, in metals, the wind force dominates electromigration. The wind force results from the momentum transfer from the current-carrying charge carriers with the defect, for an overview on calculations see the review (Sorbello, 1998) which we will follow in the next few lines. In a Drude- or Boltzmann-like model based on arguments by Huntington and Fiks (Huntington and Grone, 1961; Fiks, 1959a; and 1959b), the momentum lost by the current flow is $m v_{d} / \tau_{i}$, where $v_{d}$ is the drift velocity and $\tau_{i}$ is the collision time for electron scattering at impurity (or defect) ions. We multiply by the electron density $n$ and divide by the density of impurity ions $N_{i}$. We use the charge current $j$ to express the wind force as

$$
\vec{F}_{w}=-\frac{j / m}{e \tau_{i} N_{i}}=-e \frac{n \rho_{i}}{N_{i} \rho} \vec{E},
$$

where $\rho=E / j$ is the total resistivity and $\rho_{i}=m /\left(n e^{2} \tau_{i}\right)$ is the resistivity due to the impurity ions. Bosvieux and Friedel calculated the wind force based on the electron charge perturbation $\delta n(\vec{r})$ obtained self-consistently from the electronic wavefunctions (Bosvieux and Friedel, 1962). They separated the electron density $n(r)$ into a uniform part and a part arising from the impurity. The part from the impurity is further split

TABLE I. The activation energy barriers $E_{A}$ from the literature, see also $\mathrm{Hu}$ et al. (2003). As an example, $\mathrm{Cu}$ is chosen because of its technical relevance. Recently, there has been controversy on the activation energy barrier of surface diffusion on clean $\mathrm{Cu}$. In contrast to the values mentioned above, Choi et al. have reported a much lower value $(0.45 \pm 0.11 \mathrm{eV})$ and attributed this to the surface treatment (Choi et al., 2007). They investigated $\mathrm{Cu}$ electromigration under vacuum conditions.

\begin{tabular}{lcc}
\hline \hline Diffusion path & $\begin{array}{c}E_{\mathrm{A}}(\mathrm{eV}) \text { from } \\
\text { Hau-Riege (2004) }\end{array}$ & $\begin{array}{c}E_{\mathrm{A}}(\mathrm{eV}) \text { from } \\
\text { Lloyd (1999) }\end{array}$ \\
\hline Bulk Cu & 2.2 & 2.3 \\
Dislocations $\mathrm{Cu}$ & $1.2-1.5$ & 1.2 \\
Grain Boundaries $\mathrm{Cu}$ & $0.88-0.95$ & \\
Surface $\mathrm{Cu}$ & $0.8-0.9$ & $0.7-1.0$ \\
Interface $\mathrm{Cu}$ & & \\
\hline \hline
\end{tabular}

into an equilibrium part $\Delta n^{(0)}(\vec{r})$ and a part perturbed by the current to first order $\Delta n^{(1)}(\vec{r})$

$$
\vec{F}_{w}=-\int \Delta n^{(1)}(\vec{r}) \nabla_{\vec{R}} v_{0}(\vec{r}-\vec{R}) \mathrm{d}^{3} r .
$$

This equation includes deviations from the equilibrium electron density known as Friedel oscillations. The equation can be compared in its Fourier-transformed form to the result discussed above [Eq. (15)]

$$
\vec{F}_{w} \approx-\frac{e m \tau \vec{E}}{12 \pi^{3}} \int_{0}^{2 k_{F}}|v(q)|^{2} q^{3} \mathrm{~d} q,
$$

where $v(q)=-4 \pi Z e^{2} /\left(q^{2} \varepsilon(q)\right)$ and $\varepsilon(q)$ is the static dielectric function. $v(q)$ is the Fourier transform of the statically screened electron interaction $v(\vec{r})$ and describes the interaction of an electron with the ion and its screening cloud in the case where no current is flowing. Landauer found that a current flowing through a metallic conductor induces a localized dipole around each scatterer (Landauer, 1976). This dipole is associated with the residual resistivity. It leads to a significant contribution to the wind force that had previously been neglected and finding it thus led to a refinement of the understanding of the wind force (Sorbello, 1998).

An expression based on the Boltzmann equation was found by Das and Peierls (Das and Peierls, 1973)

$$
\vec{F} \approx Z e \vec{E}-\frac{e m \tau \vec{E}}{12 \pi^{3}} \int_{0}^{q_{c}}|v(q)|^{2} q^{3} \mathrm{~d} q,
$$

where $v(q)=-4 \pi Z e^{2} /\left(q^{2}+q_{T F}^{2}\right)$ and $q_{T F}$ is the ThomasFermi wave vector, and $q_{c}$ is a cut-off that should be on the order of $q_{T F}$. This expression contains not only the wind force as discussed above. The attribution of certain components to wind force and direct force varies in the literature. The last equation also contains contributions usually accounted for together with the direct force by other authors.

The wind force is often thought to be a property intrinsic to the material. However, it strongly depends on the microstructure of the metal and differs for ions located in the bulk, at the grain boundaries or at the surface. It depends not only on the defects in the vicinity of the ion considered, but because the electronic wave-function is delocalized, it depends also on defects further away. It also contains effects caused by multiple scattering. Measured values for $Z_{1}$ for the same metal can vary by a factor of three (Hoekstra et al., 2000). Only recently, it has been possible to measure the resistance caused by a single step on a metal surface (Homoth et al., 2009).

The wind force has been calculated both for atoms near a vacancy in the bulk (Dekker et al., 1999) as is shown in Figs. 10 and 11 and for adatoms on surfaces (Ishida, 1994; Bly and Rous, 1996; and Rous and Bly, 2000). In agreement with experiments, calculations have shown that the wind force is highly sensitive to local properties of the metal surface that depend on the local environment, e.g., on the adatom-substrate bond length and the adsorbate coverage (Ishida, 1994). An approach using the linear-response formalism of Kubo allows 


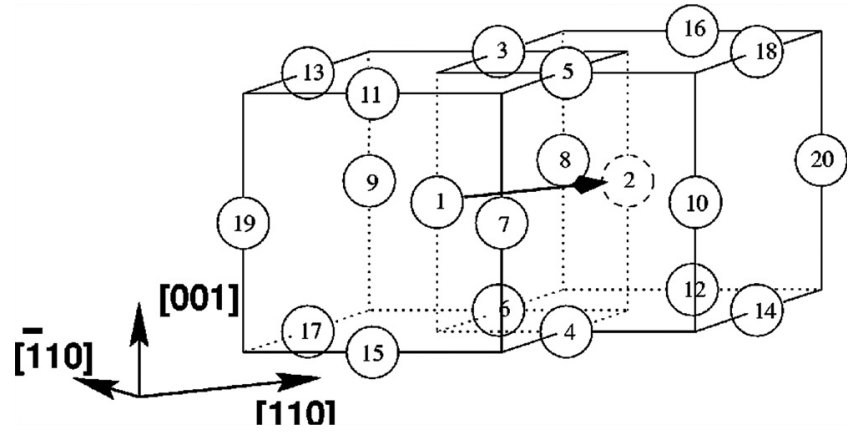

FIG. 10. The atoms in the region of the defect used for the calculated results shown in Fig. 11 are numbered. The atom 1 jumps into the original position of the vacancy 2. Reprinted with permission from Dekker et al., Phys. Rev. B 59, 7451 (1999). Copyright 1999 American Physical Society.

us to calculate the wind force for different adsorption sites (Bly and Rous, 1996). In particular, the wind force for an adatom near another adatom, atom row, island, or a step edge differs due to multiple backscattering of charge carriers (Rous and Bly, 2000). For Ag surfaces, strong scattering near defects was found to cause strong deviations from equilibrium and an experimentally observed enhanced wind force (Bevan et al., 2010 and Bondarchuk et al., 2007). For monoatomic Al nanowires, straight and zig-zag structures have been considered (Zhang et al., 2011). For the zig-zag wire, in contrast to the common case, the $\mathrm{Al}$ atoms move against the electron flow. For a $\mathrm{Si}$ atom on a carbon nanotube, it is shown that the atom stays stable regardless of the voltage applied if it is located at a position where the current does not pass underlining the necessity of a microscopic view of electromigration (Zhang et al., 2011). On the other hand, carbon nanotubes have been proposed for nanoscopic mass transport using a current (Regan et al., 2004). Also, graphene is proposed for adsorbate transport by electromigration (Solenov and Velizhanin, 2012). A recent approach proposes to scale the effective charge $Z_{1} \cdot e$

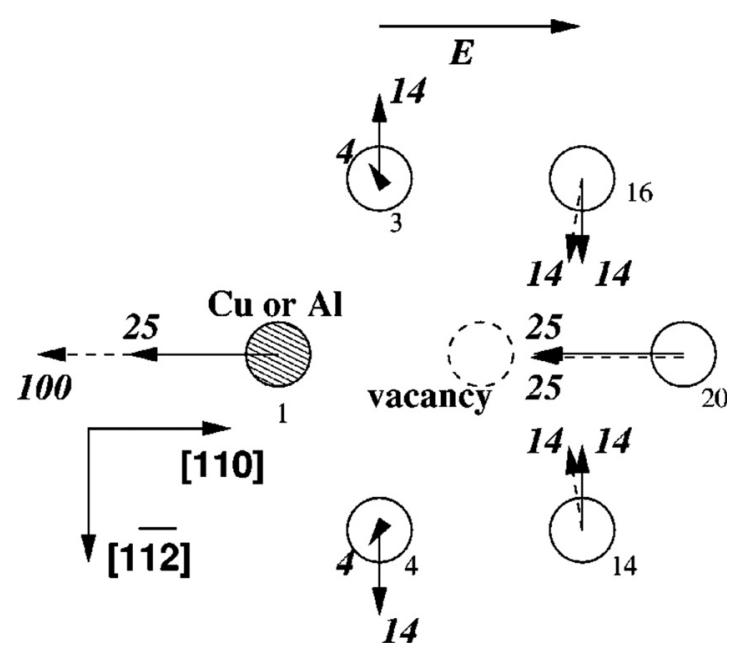

FIG. 11. The wind force for bulk atoms that are nearest neighbors of a vacancy. The solid arrows refer to calculated results for a $\mathrm{Al}$ atom at position 1 and the dashed arrows correspond to a $\mathrm{Cu}$ atom at that position. The small number give labels of the resp. atoms, and the large numbers are the magnitude of forces in $\mathrm{eV} / \mathrm{cm}$ for a current density of $1 \mathrm{MA} / \mathrm{cm}^{2}$. Reprinted with permission from Dekker et al., Phys. Rev. B 59, 7451 (1999). Copyright 1999 American Physical Society. with the size of the metallic line (interconnect) if the size of the line is below the mean free path of the metal ( $\mathrm{Wu}$ and $\mathrm{Ho}$, 2012). It is found that the size factor decreases with the size of the line due to scattering at interfaces and grain boundaries.

\section{The direct force}

The calculation of the direct force has been a controversial subject. Historically, it was first believed that the charge appearing in the direct force $Z_{2} \cdot e$ was equal to the charge of the metal ion $Z \cdot e$. Then, screening effects were recognized and, subsequently, a complete cancellation of $Z_{2} \cdot e$ was predicted, because it was believed that all electrostatic fields inside metals were fully screened (Bosvieux and Friedel, 1962). It is indeed often believed that a charged impurity and all electrostatic fields should be screened fully inside a metal. However, this cannot be correct since the same would also be applicable to an electron (Das and Peierls, 1973). This would lead to the conclusion that there is no net force on an electron inside a metal, when a voltage is applied to the metal, which is obviously wrong. Today, we describe electron transport by the Boltzmann equation which links the electrostatic force and the electronic non-equilibrium states. Das and Peierls and Landauer have provided semi-classical expressions for the direct force (Das and Peierls, 1973 and Landauer, 1976). In this approach, the idea is that screening causes the density of carriers to be increased in the vicinity of a positively charged impurity. For the current to remain continuously at the same level, the electrostatic field must then decrease to compensate the increased conductance in the region. As a result, the net effective valence is reduced (Landauer, 1976). One obtains for the direct force

$$
F_{d}=\frac{Z e E}{1+(1 / 3) \beta \Delta n / n_{0}},
$$

where $\Delta n$ is the local increase in carrier density due to the impurity, $n_{0}$ is the average electron density in the metal, and $\beta$ is a parameter of order unity. This approach still lacks information about the atomic wave functions and their polarizability. A linear-response expression including both the direct and the wind force was obtained (Kumar and Sorbello, 1975). The evaluation of this expression depended on the potential used for the ion and gave a screening of 10\%-30\% (Sorbello, 1985). This is in agreement with a more recent approach, where a screening of $5 \%-25 \%$ is found (Lodder, 2005). The electrostatic fields near impurities have been recalculated using first-principles calculations and were found to be very intense due to the short-range nature of the screening in metals (Bevan et al., 2012).

\section{Combined approaches}

Some authors have favored an unseparated approach to both quantities, the direct and the wind force (Hoekstra et al., 2000). Which contribution to the force is regarded as a wind force and which is regarded as a direct force is a matter of interpretation (Sorbello, 1998). An intuitive derivation of five different types of forces on nuclei in conductive systems intended to be understandable at the undergraduate level has 
been given (Todorov et al., 2014). It identifies the two forces acting only in the presence of a current, the wind force, and a Lorentz-like velocity-dependent force, see also Bode et al. (2011). The current-induced forces arise from a redistribution of the population of different electronic orbitals with bonding and antibonding character (Brandbyge et al., 2003).

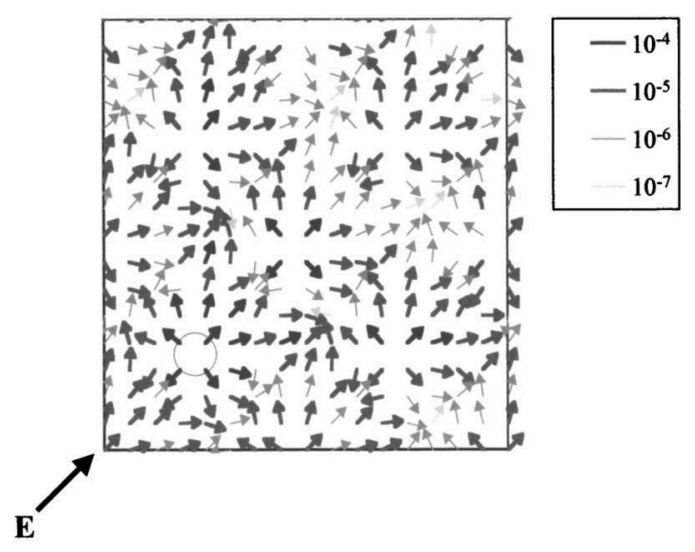

(a)

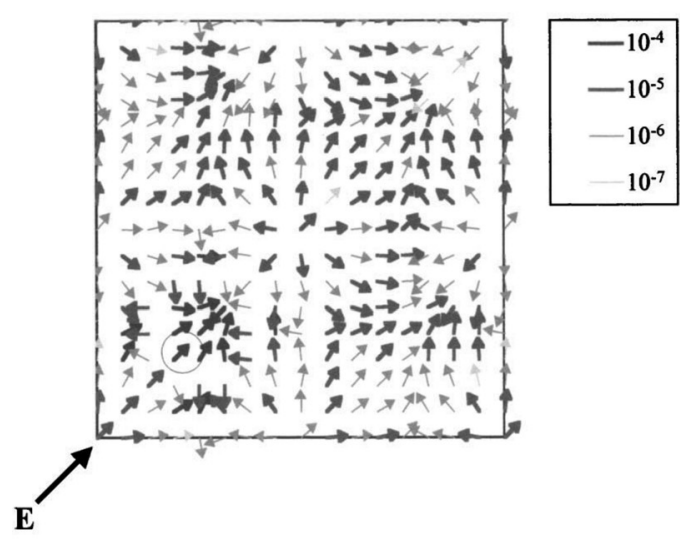

(b)

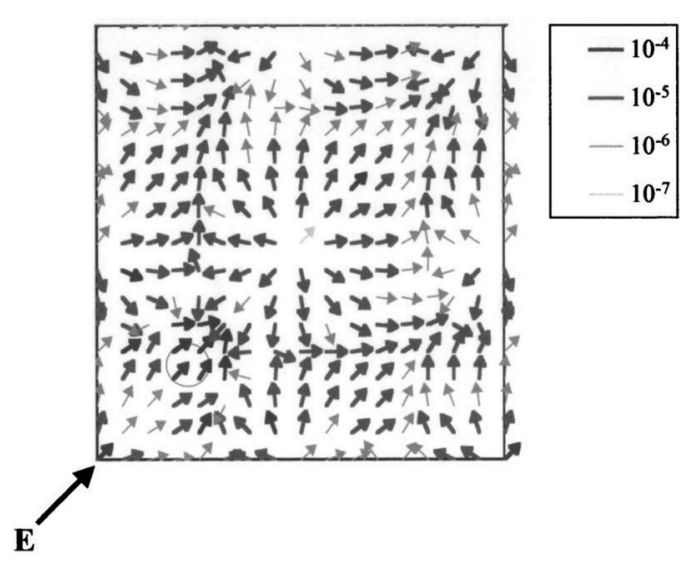

(c)

FIG. 12. Maps of the electromigration force density, for (a) bulk, (b) surface, and (c) grain boundary. The open circle shows the wave-packet core of the moving particle. Reprinted with permission from Doi et al., Phys. Rev. B 67, 115124 (2003). Copyright 2003 American Physical Society.
It is important to consider dynamic effects, since the electromigration forces can only be understood by the coupling of electronic and atomic motion. The total currentinduced forces have been calculated in a picture where the moving atom was considered a quantum-mechanical object and dynamic effects have been considered (Doi et al., 2003), see Fig. 12. The figure shows that the total force is not constant at all as a function of lateral position but changes strongly in direction and magnitude depending on the local position and environment. Current-induced forces are nonconservative (Cunningham et al., 2014) and can therefore in principle be used to drive another atomic object (Dundas et al., 2009 and Fernández-Alcázar et al., 2015).

For electromigration forces, electron-hole asymmetries and/or vacancy-atom asymmetries are of special interest. In a metal with perfect electron-hole and vacancy-atom symmetry, the thermalization of hot electrons in the anode and the thermalization of hot holes in the cathode would be identical (Lee et al., 2013). For vacancies and atoms, there is a trivial difference in that metal atoms can carry a current whereas accumulated vacancies are voids that do not carry a current. In addition, $Z_{1}$ and $Z_{2}$ could differ for electron transport and hole-like transport. Recently, another vacancy-atom asymmetry has been put to the center of the discussion: rotational vibrational modes differ for atoms and vacancies because atoms have a considerable mass and moment of inertia, whereas vacancies do not have such a property. This could lead to scattering properties that differ for electrons and holes and consequently to asymmetric heating of the electrodes. Experimental studies on asymmetric heating will be discussed in Sec. VI A. Runaway vibrational modes can lead to a circular motion of atoms occurring deterministically at specific threshold voltages by quantum effects (Lü et al., 2010). The relative importance of heating and current-induced forces as described in a toy model in Fig. 6 has been explored (Yang et al., 2005). It is found that low-bias instabilities of the wires are mainly caused by local heating while failure at high biases is dominated by current-induced forces. In another study, this aspect was also stressed (Todorov et al., 2001): the current-induced forces are not sufficient to break an atomic Au chain, but they reduce the work needed to break the chain such that it can break by thermal activation. Other studies find that the forces should be sufficiently large (Brandbyge et al., 2003) Indications for such deterministic breakage at low voltages have been found experimentally in Au mechanically controlled break-junctions (Sabater et al., 2015). Efforts have been taken to measure forces in the presence of a tunneling current. Mostly, well-known forces such as the Pauli repulsion force (Weiss et al., 2010 and Zhang et al., 2011) and electrostatic forces (Weymouth et al., 2011) have been found. Also, the force noise under the influence of a current has been studied (Herz and Scheer, 2016).

\section{E. Using electromigration to generate metallic nanocontacts}

In order to use electromigration procedures to generate metallic nanocontacts, the simplest approach is to fabricate a 

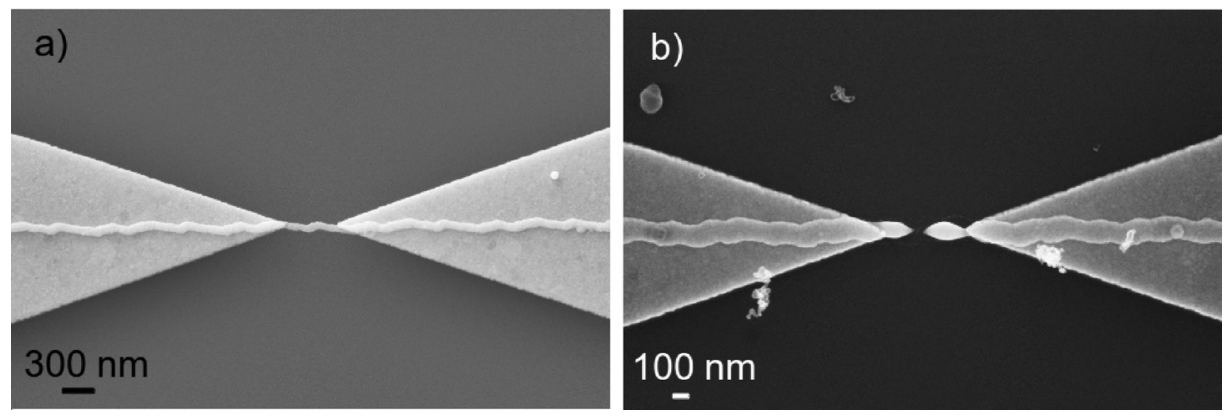

FIG. 13. Thin metallic Au contact with large triangular electrical connections before (a) and after (b) uncontrolled electromigration. Heating has led to melting of the wire (R. Hoffmann, D. Weissenberger, J. Hawecker and D. Stöffler, unpublished).

nanobridge and to ramp up the voltage until the wire breaks (Park et al., 1999 and Bolotin et al., 2004). The yield for nanocontacts produced in this way is low, because there is a considerable danger that the wire could melt, as e.g., in Fig. 13.

As will be explained in detail below, in the region shown in red in Fig. 14, the wire is heated to temperatures where electromigration proceeds uncontrollably fast. It becomes clear from the figure that choosing a constant voltage of $0.4-0.6 \mathrm{~V}$ could severely damage the nanobridge since this process would mean to move on a vertical line in the figure, passing through the red area where the wire melts. In the worst case, the nanobridge could melt, as e.g., in Fig. 13. In such a process, the yield of unmolten samples can be increased by minimizing the lead and any contact resistances, because this changes the shape of the dashed red line given by Eq. (22) (Trouwborst et al., 2006). As a further development, one can eliminate the lead resistance by using a four-terminal geometry (Wu et al., 2007). However, this is not always possible, as has been pointed out, e.g., in Campbell and Knobel (2013). It has been stressed that for both controlled and uncontrolled electromigration, the series resistance should be small to obtain small gaps (Taychatanapat et al., 2007).

The nanobridge can be fabricated by various techniques. The best known techniques are electron-beam lithography and ion milling. The precise shape of the nanobridge influences the electromigration characteristics. If the shape of the nanostructure is irregular, e.g., it results from two overlapping evaporation steps, a flux divergence in the diffusion of

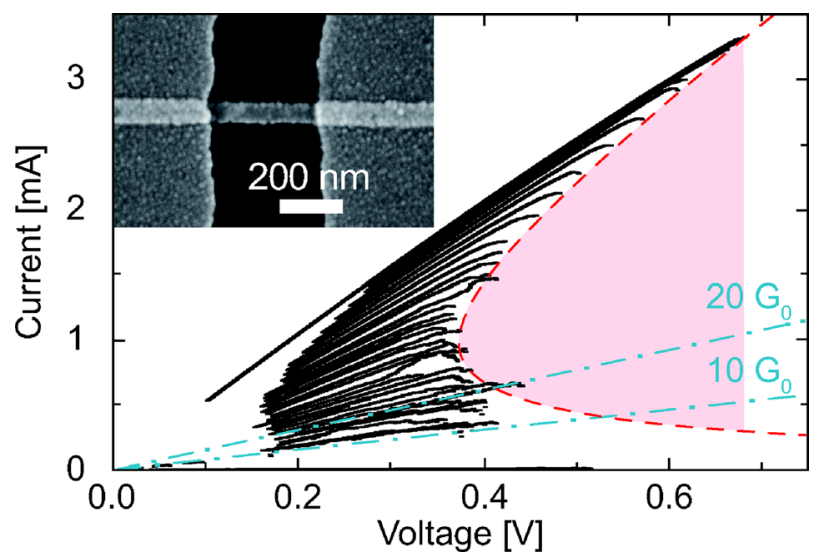

FIG. 14. Current as a function of total voltage $U$ during the electromigration procedure (black data-point traces). The dashed red line shows a constantpower curve with $P^{*}=0.18 \mathrm{~mW}$ and a lead resistance of $R_{L}=188 \Omega$. Dashed-dotted blue lines correspond to conductances of 10 and $20 G_{0}$. Inset: SEM image of a nanowire. Reprinted with permission from Hoffmann et al., Appl. Phys. Lett. 93, 043118 (2008). Copyright 2008 AIP Publishing, LLC. atoms can occur and enhance electromigration failure as will be discussed in more detail in Sec. III B. Therefore, one should carefully choose the shape of the nanobridge.

\section{Controlled electromigration}

After a long history of studying electromigration at a constant temperature of the environment, it became clear that due to the strong temperature dependence of the process, it is necessary to study electromigration at a constant temperature of the contact itself. This became possible by using the resistance of the contact itself to measure its temperature. Due to the metallic conduction mechanism, the resistance of the contact rises roughly linearly as a function of temperature around room temperature

$$
R(T)=R_{0}\left(1+\alpha\left(T-T_{0}\right)\right),
$$

where $R_{0}$ is the resistance at the temperature $T_{0}$ and $\alpha$ is a temperature-independent coefficient. After applying a voltage, the contact is heated through Joule heating and its temperature rises above that of the environment. For diffusive conductors for which the Wiedemann-Franz-law $(k / \sigma$ $=L \cdot T$ ) holds, i.e., where current and heat follow the same path, we can assume that electronic conduction (electrical conductivity $\sigma$ ) is related to heat conduction (thermal conductivity $k$ ) and this leads to the relation (Holm, 1967 and Halbritter et al., 2002)

$$
L_{0}\left(T^{2}-T_{0}^{2}\right)=\frac{U_{C}^{2}}{4},
$$

where $L_{0}=\left(\pi^{2} / 3\right) \cdot\left(k_{B} / e\right)^{2}$ is the Lorenz number, $T$ and $T_{0}$ are the temperatures of the contact and its environment, respectively, and $U_{c}$ is the voltage applied to the contact. This relation emphasizes that temperature is a monotonic function of voltage. We therefore need to control the voltage in a precise way if we aim at controlling the temperature in a precise way. However, since the resistance of the contact increases during the thinning process, it is difficult to distinguish a resistance increase due to thinning and a resistivity increase due to heating. By computer-control, it became possible to ramp up the voltage in cycles to solve this problem (Shih et al., 2003; Esen and Fuhrer, 2005; Strachan et al., 2005; and Hoffmann et al., 2008). In each cycle, the contact and lead total resistance at low voltages is assumed to be constant and can be measured. Then, the voltage is slowly increased. At larger voltages, the contact resistance increases due to heating and electromigration takes place at an 
enhanced speed. When the resistance increases by a preset amount, the voltage is immediately switched off or strongly reduced and a new cycle is started from low voltages. Thus, the voltage and the temperature of the contact are limited, and we call this process "controlled electromigration," while the microscopic process of electromigration remains uncontrolled. A typical resulting curve consisting of many $I(U)$ cycles is shown in Fig. 14.

The characteristic shape of the curve can be easily explained. It is useful to compare with a line of constant power dissipated in the nanocontact $P^{*}$. We assume that the device consists of leads with a constant resistance $R_{L}$ and of a contact with a resistance $R_{C}$. $R_{L}$ results from grain-boundary scattering and from scattering at the surfaces of the thin film, as, for example, described by the Fuchs-Sondheimer model or similar models (Sondheimer, 1952 and Durkan and Welland, 2000b). $R_{C}$ increases during the thinning process as the nanocontact is formed. We can write for the total voltage $U$

$$
U=U_{C}+U_{L}=P^{*} / I+R_{L} \cdot I
$$

where $U_{C}$ is the voltage drop at the contact, $U_{L}$ is the voltage drop at the leads, and $I$ is the current. Such a curve gives the dashed red line in Fig. 14. In the region to the left of this curve, the dissipated power is smaller than the value used to generate the curve, and in the region marked in red to the right of the dashed red line, this power is larger. Consequently, the contact can become hotter than desired in the region marked in red which should therefore be avoided to avoid melting. One can immediately see that it is difficult to avoid the red region if one works at constant voltage, because the resistance of the leads varies and thus it is not clear beforehand which voltage to choose. In addition, even for voltages larger than the critical one $(0.37 \mathrm{~V}$ for the contact in Fig. 14), the temperature of the contact at the start of the process is low and electromigration proceeds only at long timescales.

In Fig. 14, most of the end points of the individual cycles lie indeed on or near the constant power curve. This shows that the resistance change is a smooth function of temperature (ideally, a linear function) and that both are a smooth function of the power dissipated in the contact.

Deviations from this relationship occur in the region below 10-20 conductance quanta $G_{0}$, when ballistic effects become important. These ballistic effects will be covered in Sec. IV.

Controlled electromigration offers a new view on spectroscopic studies of the binding energies, because ideally studies could be performed at a constant temperature at the contact itself. Within this simple model, we associate the temperature scale with an activation energy barrier scale. Using Eq. (21) or similar, we can furthermore associate this temperature scale with a voltage scale. At low voltages, atoms or defects with the smallest activation energy barrier will become mobile in the contact region, move out of the contact due to diffusion, and redeposit in cooler regions.

\section{Temperature estimates}

The process of electromigration is closely linked to the local temperature distribution in the device, because it is
TABLE II. The voltages and temperatures when contacts start to change their structure at room temperature. Metals with larger melting temperatures are also stable at larger voltages. Note that these values were obtained in the case where the lead resistance is negligible. From Holm (1967).

\begin{tabular}{lcccc}
\hline \hline & $\begin{array}{c}\text { Softening } \\
\mathrm{T}\left({ }^{\circ} \mathrm{C}\right)\end{array}$ & $\begin{array}{c}\text { Melting } \\
\mathrm{T}\left({ }^{\circ} \mathrm{C}\right)\end{array}$ & $\begin{array}{c}\text { Softening } \\
\mathrm{U}(\mathrm{V})\end{array}$ & $\begin{array}{c}\text { Melting } \\
\mathrm{U}(\mathrm{V})\end{array}$ \\
\hline $\mathrm{Ag}$ & 180 & 960 & 0.09 & 0.37 \\
$\mathrm{Al}$ & 150 & 660 & 0.1 & 0.3 \\
$\mathrm{Au}$ & 100 & 1063 & 0.08 & 0.43 \\
$\mathrm{Cu}$ & 190 & 1083 & 0.12 & 0.43 \\
$\mathrm{~Pb}$ & 200 & 327 & & 0.12 \\
$\mathrm{Pd}$ & & 1552 & & 0.57 \\
$\mathrm{Pt}$ & 540 & 1772 & 0.25 & 0.71 \\
\hline \hline
\end{tabular}

enhanced by thermal activation. In the diffusive regime, scattering dominates and the local resistivity is well-defined. A simple estimate of the temperature is obtained using Eq. (21). Using this equation, a measurement of the voltage at which the contact starts to transform gives a rough estimate of the temperature. This temperature is not always related to the melting temperature of the metal used as can be seen from the table extracted from Holm (1967) (Table II).

\section{Time-scale of controlled electromigration}

Due to the view on electromigration as a selfaccelerating process, one expects that a precise temporal control was necessary as is suggested by the Black equation [Eq. (4)]. However, if the temperature is maintained at sufficiently small values, the precision needed on the temporal control remains reasonable. The process can thus usually be slowed down to the requirements of the experiment by the control of the temperature at the nanocontact itself as can be seen, e.g., from Fig. 14. While atomic jumps remain fast and happen at the timescale of phonon frequencies, the jumping rate can be on the timescale of seconds (Hoffmann et al., 2008).

\section{ELECTRONIC CHARACTERIZATION METALLIC NANOCONTACTS-IMPLICATIONS FOR THE STRUCTURE}

The electronic properties of nanocontacts show interesting quantum effects and have been reviewed (Agraï et al., 2003). The purpose of this review is to focus on the structural properties of nanocontacts. Often, the electronic properties provide implications for the structure of the nanocontacts. In many cases, they remain the only source of information on the structure of a particular type of nanocontacts.

\section{A. Macroscopic part, thermal regime}

\section{Initial stages of current flow through freshly prepared samples}

Most of the methods to study the structure or electronic properties of nanocontacts, such as the mechanically controlled break-junction method or the electromigration method, rely on thinning of relatively large (on the order of 
$10 \mathrm{~nm}-1000 \mathrm{~nm}$ thick or wide) metallic bridges. Here, we discuss effects observed when these bridges are subject to a large current density for the first time.

From the discussion above as well as from early studies, we expect that in the region of the forming nanocontact, first a region of increased resistivity is formed. However, in the initial stages of controlled electromigration instead of an increase, we often see a decrease of the resistance (Stöffler and Hoffmann-Vogel, 2015): as can be seen from Fig. 15 the red line describing the resistance, the first electromigration cycle, lies above subsequent cycles, the black lines. Also, for wires in the ballistic regime, an initial enhancement of conductivity due to electromigration is observed (Schirm et al., 2013 and Strigl et al., 2015). We attribute this decrease to annealing of the contact. For thin-film conductors, during deposition of the metal, defects are buried in the metal. Due to heating, these defects reach their activation energy barrier at lower temperatures compared to the undisturbed bulk. The defects could be healed or diffuse along the wire.

\section{Size of the heated region}

Above we have described how hot-spots form during electromigration as the wire is thinned (Sec. III A). The formation of hot-spots also has an effect on the $I(U)$ characteristics observed during cyclic electromigration. It has been shown above that the function connecting the end points of the electromigration cycles reached with a preset resistance change is a hyperbola of constant dissipated power in the contact. From the values of this hyperbola, one can obtain information about the power needed to sufficiently heat the junction in order to thermally induce electromigration. It is plausible to assume that if more power is dissipated at the onset of electromigration, the size of the heated region must be larger. The size of the heated region may change in the course of the experiment. In particular for hot-spot formation, we expect that the size of the heated region and thus the dissipated power become smaller during the experiment, as has been observed, see Fig. 16(a) and Stöffler et al. (2012) and Girod et al. (2012).

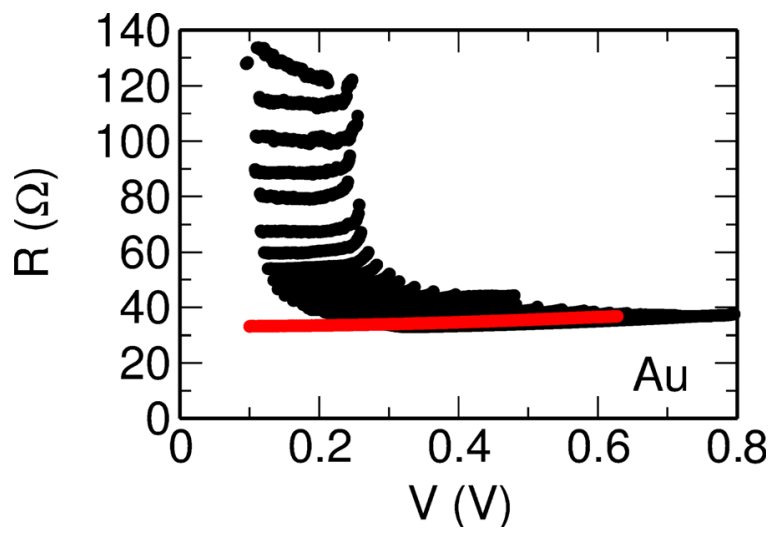

FIG. 15. When a Au thin film is thinned by a controlled electromigration process, the resistance decreases after the first electromigration cycle (marked in red) and then continuously increases from cycle to cycle (data marked in black). Other aspects of the data will be discussed in detail below (Fig. 22). Reprinted with permission from Stöffler et al., Phys. Rev. B 90, 115406 (2014). Copyright 2014 American Physical Society.
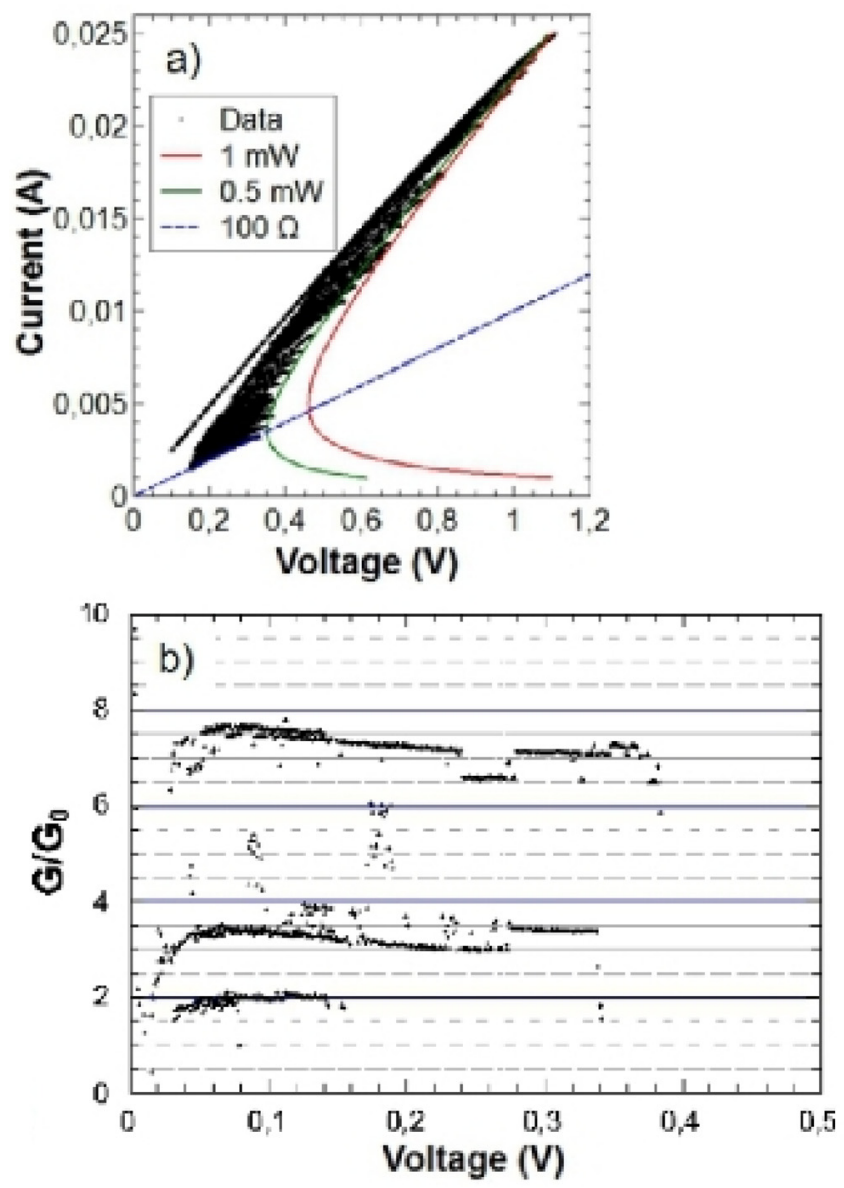

FIG. 16. (a) Overall electromigration process of a Au thin film with two lines of constant dissipated power as a guide to the eye. The behavior switches permanently from a larger power to a smaller power. This follows because the red hyperbola [a line of constant power dissipation, see Eq. (22)] follows larger voltages and currents than the green hyperbola. (b) In the region where the conductance has values comparable to small multiples of the conductance quantum $G_{0}$, repeated switching between several conductance values is observed. The switching is presumably related to atomic jumps. Reprinted with permission from Stöffler et al., Phys. Rev. B 85, 033404 (2012). Copyright 2012 American Physical Society.

\section{Several weak spots in one metallic conductor}

When the metallic bridge is thinned down by electromigration, it is possible that several weak spots in one metallic conductor are formed. For fundamental studies, it is important to be able to detect such situations in order to correctly interpret the measured results. Several weak spots easily occur in a network of interconnects as is used on computer chips. The behavior of networks of interconnects therefore has important implications for the semiconductor industry.

If there are two weak spots in series, one of them will in general show a larger resistivity than the other, and therefore, the local temperature at this spot will be higher since both carry the same current. The strong electromigration at the hotter spot will cause the resistivity to increase even more at this position, and therefore, this hot-spot will dominate the behavior of the metallic conductor. This is also the reason why electromigration is expected to occur only at one position even for a wire with two weak spots in series. However, the size of the heated region can be large, and therefore, 
inside the heated region changes can be observed at several positions, and this will be discussed below in the section on structural characterization.

Two weak spots could be arranged in parallel. If spot 1 has a larger resistivity, the current will primarily run over spot 2 , and spot 2 will thus be heated with more power and its resistivity will become even larger until its resistivity is larger than that of spot 1 , and we expect an oscillation between dominant behavior of the two different spots. Oscillating behavior of a more complex origin is shown in Fig. 17. Controlled electromigration of networks of contacts has been studied (Johnston et al., 2007). It was found that it is indeed possible to fabricate a number of devices in parallel using one electromigration-thinning procedure. For such a process to be successful, it is important to either add a series resistance common to both nanobridges or introduce shorts between the nanobridges. This is in agreement with the electromigration of ring structures where both arms of the rings were electromigrated at a similar pace (Kiessig, 2012).

\section{B. Ballistic regime}

\section{Overview}

When a metallic contact is thinned continuously, for example, using a mechanically controlled break-junction, at some point the resistance no longer decreases smoothly as expected from the size dependence of the macroscopic resistance

$$
R=\rho \cdot \frac{l}{A},
$$

where $R=G^{-1}$ is the resistance, $\rho$ is the resistivity, $l$ is the length of the conductor, and $A$ is its cross-section. Instead, the resistance changes discontinuously.

These jumps are related to both the atomistic nature of the metal and the quantum-mechanical wave nature of electrons. The properties of the metallic conductor could be viewed as those of a waveguide for electrons having the Fermi wavelength $\lambda_{F}$ (Agrait et al., 2003). In this case, only a finite number of modes can propagate in the waveguide.

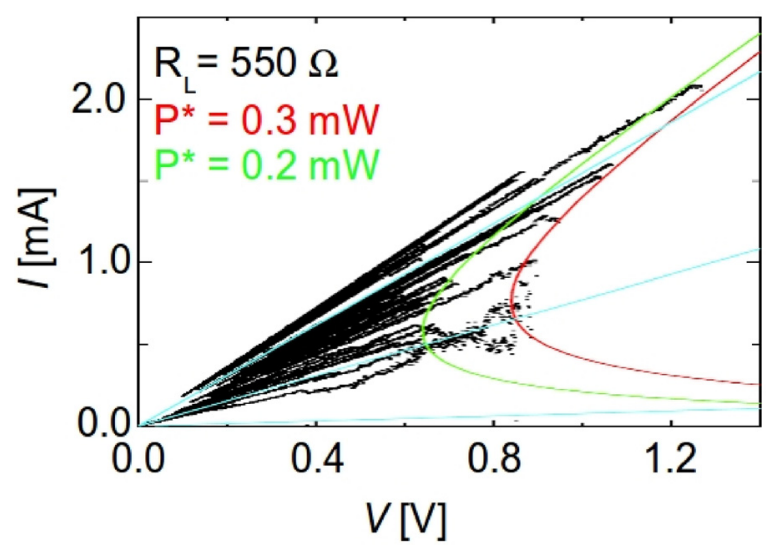

FIG. 17. Electromigration process of a Au thin film with two different lines of power showing an oscillation between dominant behavior of each of the two power values. Reprinted with permission from R. Hoffmann, D. Weissenberger, J. Hawecker and D. Stöffler (unpublished).
From the wave nature of the electron, one expects that one wave-guide mode ("channel") contributes at most $G_{0}=2 e^{2} / h$, the conductance quantum, to the conductance for perfect transmission. Depending on the relation of the size of the waveguide and the Fermi wavelength, the conductance is expected to increase in steps as the size of the junction increases continuously. This has been observed first for semiconductors, where due to the small carrier density $\lambda_{F}$ is much bigger than the size of an atom (van Wees et al., 1988 and Wharam et al., 1988). Indeed for metals thinned with mechanically controlled break junctions, one observes jumps on the order of the conductance quantum upon thinning, see Fig. 17. On the other hand, for metals, the size of the contact cannot be changed continuously in the range of the Fermi wavelength, due to the atomistic nature of matter and because here $\lambda_{F}$ is approximately equal to the size of an atom. Jumps are related to discontinuous displacements of atoms that also change the electronic properties of the contact (Yanson and van Ruitenbeek, 1997). In fact, both aspects are closely related, not only because the Fermi wavelength is on the order of the size of an atom, but more intrinsically, because the electronic states are formed by the electronic wavefunctions of the atoms in the contact.

Both aspects are summarized in the Landauer equation (Landauer, 1970)

$$
G=G_{0} \cdot \sum_{i, j} \tau_{i j},
$$

where $G_{0}=2 e^{2} / h$ is the conductance quantum describing the conductance of one electronic waveguide mode and $\tau_{i j}$ is the transmission probability of the respective input and output channels $i$ and $j$. The Landauer equation is not only valid in the ballistic regime but becomes particularly important in this regime. The transmission probability strongly depends on the electronic wavefunction overlap of the atoms in the contact (Scheer et al., 1998). With the help of this equation, the conductance jumps can be interpreted as a result of a small number of $\tau_{i j}$ with values substantially larger than zero that suddenly change upon opening or closing the junction and all other $\tau_{i j}$ with negligible values. These jumps and changes in the values of the $\tau_{i j}$ are the key to obtain structural information from electronic transport measurements.

The regime where electronic wave properties become important is called the ballistic regime, because the contact in this regime becomes smaller than the mean free path of scattering. The electrons are thus transmitted through the contact without scattering and are therefore in a nonequilibrium state. Due to this non-equilibrium, it is a problem to define the local resistivity $\rho$.

Such jumps in the conductance are also observed for STM indentation methods. For metallic nanocontacts generated by controlled electromigration, also discontinuous jumps are observed at conductances on the order of the conductance quanta, see Figs. 16(b) and 18. The different levels correspond to different atomic configurations the contact reaches by thermal hopping of atoms. Such steps have been observed, e.g., for Au (Hoffmann et al., 2008 and Strachan et al., 2005) and for Bi (Sangiao et al., 2013). 


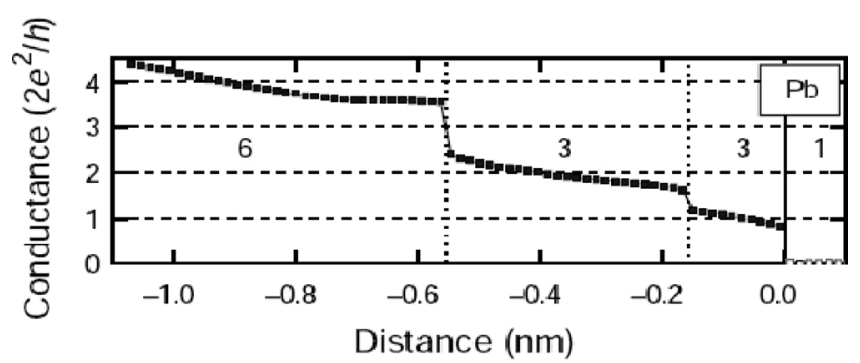

FIG. 18. Conductance as a function of electrode distance for a $\mathrm{Pb}$ breakjunction. The numbers denote the minimum number of channels necessary to explain the multiple Andreev spectra measured on the respective plateaus. Reprinted with permission from Nature 394, 154 (1998). Copyright 1998 Macmillan Publishers Limited.

\section{Histograms generated from electromigration studies}

In the ballistic regime, the conductance of the devices is studied in histograms to obtain the number of observed conductance values in a conductance bin. Atomic configurations that are rather stable are observed more frequently and therefore have a pronounced weight in the histogram. These histograms are mostly generated from mechanically controlled break-junctions or from STM devices. In order to obtain sufficient statistics, a contact is formed and broken a large number of times (10000-100000). All the data points of the conductance measured in a given time interval are taken to form a histogram. It has been found that the details of these histograms and in particular the number of peaks depend on a variety of conditions: the material used to make the wire, its microstructure, whether the histograms are obtained during opening or closing, the voltage applied (Nielsen et al., 2003), the temperature (Yanson et al., 2005), the pressure and nature of the residual gas (Untiedt et al., 2004), and the intervals of conductance and time bins chosen. For an overview, see Agrait et al. (2003). For Au and monovalent metals such as alkali metals, 10 or more conductance peaks are observed, whereas for magnetic materials such as $\mathrm{Fe}$, Co, and $\mathrm{Ni}$ even for good statistics and pure cryogenic vacuum conditions, only two or three peaks in histograms are observed.

A simple method to generate histograms for cyclic electromigration methods is to take the full data set of conductance obtained during thinning from the chosen voltage and measured current values. Such a histogram is shown in Fig. 19. With this method, many measurements are counted for the same value of the conductance, because the measurement of the same state is repeated for different voltages in the voltage ramp. This naturally leads to large peaks for one particular conductance value. Similar to mechanically controlled break junctions, also the height of the peak depends on the stability of the atomic configuration. Usually, the statistics is much lower because it is technically out of question to prepare 10000 or 100000 individual samples by electromigration. For such histograms, many more peaks are observed and it is at first not clear which of them occur due to statistical fluctuations and insufficient averaging. Credence to the histogram is obtained by observing that the peaks in Fig. 19 can be compared with peaks obtained from the break-junction method,

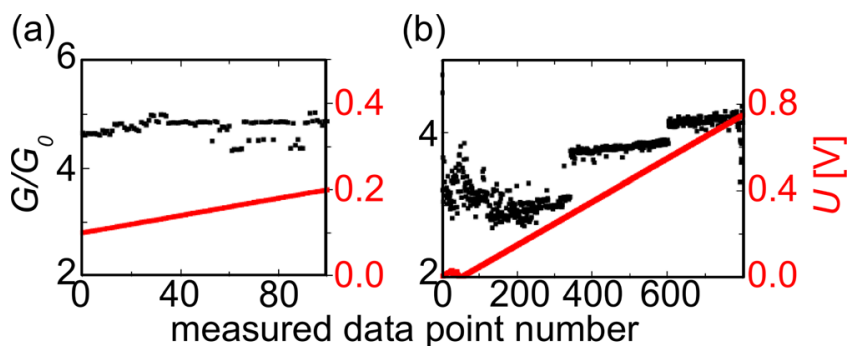

FIG. 19. Thermal jumps of atoms between different configurations having conductances on the order of a conductance quantum. The data are taken during electromigration. As a consequence due to the controlled electromigration process, the voltage increases as a function of time as shown by the data indicated in red. Reprinted with permission from Hoffmann et al., Appl. Phys. Lett. 93, 043118 (2008). Copyright 2008 AIP Publishing, LLC.

denoted by red lines, and there is a good agreement between the peaks found by the two methods.

An important difference between the two preparation methods is that in mechanically controlled break-junctions, the peaks are obtained from mechanically pulling the contact, which puts the contact in a non-equilibrium situation by applying mechanical force (Cross et al., 2006). In addition, often low temperatures are used for better results of the electron transport measurements and for cleanliness. In contrast, for controlled electromigration, the atoms reach their respective positions through heating and chances are larger to reach an equilibrium atomic configuration. Therefore, we expect that in general sharper and more peaks are observed compared to histograms generated by the mechanically controlled break-junction method. On the other hand, results obtained from several voltages are mixed in one histogram and consequently voltage-dependent effects could be hidden.

\section{Self-breaking as observed in the electronic properties}

An alternative method of studying the influence of the atomic configuration on the conductance of the nanoscale metallic junction is to subject the contact to a pre-chosen voltage and to observe the conductance as a function of time (Brouër et al., 2001; Ohnishi et al., 1998; Muller et al., 1996; and Ralls et al., 1989). Such studies have been performed using various techniques, e.g., evaporation of metallic films at low temperatures, where amorphous layers are obtained that contain a large number of defects (Brouër et al., 2001). In another study, a hole of $40-100 \mathrm{~nm}$ in diameter in a $\mathrm{Si}_{3+x} \mathrm{~N}_{4-x}$ membrane was fabricated and filled with $\mathrm{Cu}$ or $\mathrm{Al}$ (Ralls et al., 1989). Also, the break-junction technique (Muller et al., 1996) and TEM observation were used (Ohnishi et al., 1998).

In these experiments, the voltage is strongly reduced compared to the maximum values used during controlled electromigration cycles, and electromigration is thus strongly slowed down. In most of these studies, it is not easy to know whether electromigration is still active at the voltages applied. Due to the residual current flow, we expect a residual wind force acting on the wire; however, this is a subject of further study. In the conductance, often different conductance levels can be discerned that change as a function of time. If two levels are dominant, telegraph noise is observed 
resulting from the two different configurations, see Fig. 18(a). The telegraph noise can be discerned clearly from other sources of noise such as shot noise due to the quantized nature of charge or local vibrational modes (Wheeler et al., 2013). From the hopping rate observed in the telegraph noise, the energy barrier can be estimated. If the residence time in both states is equal, the two states must be degenerate and electromigration is inactive for these states.

During such measurements at small voltages, after a long time of little permanent change of the conductance, suddenly the contact breaks. From this initial long time, a large peak is produced in the histogram. In addition, for $\mathrm{Au}$, usually a peak at $1 G_{0}$ and a peak at $1.8 G_{0}$ are observed; the position of additional peaks seems to depend on the fabrication method of the nanocontact and the chemical environment of the setup during the measurement (Hauser et al., 2016; Kiguchi et al., 2007; Bakker et al., 2002; and Hansen et al., 1997). The breakage can be understood by the evolution of stress in the sample, as will be discussed in the following, see Sec. IIIB. The stress builds up due to electromigration-induced hopping of atoms over relatively long time-scales and then leads to failure.

Also, thermal atomic hops can lead to failure, as it was found that the contact broke even after removing the applied voltage, when the conductance was in the range of a few conductance quanta (O'Neill et al., 2007). The time needed for formation of separate electrodes was in rough agreement with the diffusion constants obtained from STM measurements. In mechanically controlled break-junctions, initial stretching had a profound influence on the subsequent selfbreaking (Tsutsui et al., 2008). The contacts recover from the applied stress by structural rearrangements. Only for extremely low stretching rates, the junctions demonstrate a static thermally activated breakdown.

\section{The structure of nanoscale metallic junctions in the ballistic regime}

When the conductance of nanoscale metallic junctions is studied with methods that do not allow to study their structure at the same time, one relies on statistics of a large number of contacts, as in conductance histograms. The prominent features in the conductance histograms need an explanation which is often sought in a particular structure of the contact that is energetically more favorable than other structures. Concerning the stability of the metallic nanowire's structure at high current densities, both experimental and theoretical results are known from the literature: For Pt, it has been shown that monoatomic chains form, but a large current density tends to destabilize these monoatomic wires (Nielsen et al., 2003). Similar results were found for $\mathrm{Al}$ and $\mathrm{Au}$, but the transition was observed to occur at high voltages $(0.8 \mathrm{~V}$ and $2.4 \mathrm{~V}$ ) compared to Pt (0.3-0.4 V) (Mizobata et al., 2003). It has been shown by calculations that metallic wires with "magic" conductance values are stable under large current densities (Zhang et al., 2005). The word magic is used here to describe shell effects in analogy with the magic numbers of atoms of few-atom clusters in the gas phase.
For thicker wires, two types of shell effects are observed, electronic and atomic shells, also found in TEM studies, as will be discussed in Sec. V. For electronic shell effects, the electronic energy has a strong influence on the total energy of the nanowire and this energy is minimized when a closed shell of electrons can be formed. Atomic shells in contrast correspond to a closed shell arrangement of the atomic configuration. For alkali metals such as $\mathrm{Na}$, electronic shell effects are observed in particular for small diameters of the nanowires (Yanson et al., 1999 and Yanson et al., 2000). For larger diameters, there is a cross-over and atomic shell effects dominate. Similar observations are made for $\mathrm{Au}$ (Mares et al., 2004 and Yanson et al., 2001). Electronic and atomic shell filling was observed for $\mathrm{Ag}$, while for $\mathrm{Cu}$, only electronic shell filling was found (Mares and van Ruitenbeek, 2005).

For $\mathrm{Au}$, compared to annealed $\mathrm{Au}$ wires, additional peaks were found in the histogram for work-hardened $\mathrm{Au}$ wires. These peaks were identified as the signature of different crystallographic orientations of the main axes of $\mathrm{Au}$, namely, [111], [001], and [110] orientations (Yanson et al., 2005), see also Fig. 20. The conclusion was drawn that the work-hardened wires contain more defects which allow for more mechanical freedom to obtain such low-energy configurations. Peaks corresponding to the [110] orientation in line with the previous study were also found in histograms of [110]-oriented $\mathrm{Au}$ wires investigated with TEM (Kurui et al., 2007 and Kurui et al., 2009). By investigating the arrangement of the atoms in the wire at the same time as the conductance, it was shown that a characteristic atomic arrangement was observed at each peak such as an atomic sheet or a hexagonal prism (Kurui et al., 2009). By comparison with first-principles calculations, it was shown that the conductance channels were opened one by one.

General relations of the conductance as a function of radius of the contact or nanowire $R$ allow us to gain estimates of the contact structure. In the thermal regime, the Maxwell relation for a metal-filled infinitely large volume cut into two parts by a thin insulating layer with a circular opening is derived from classical electrodynamics (Maxwell, 1891)

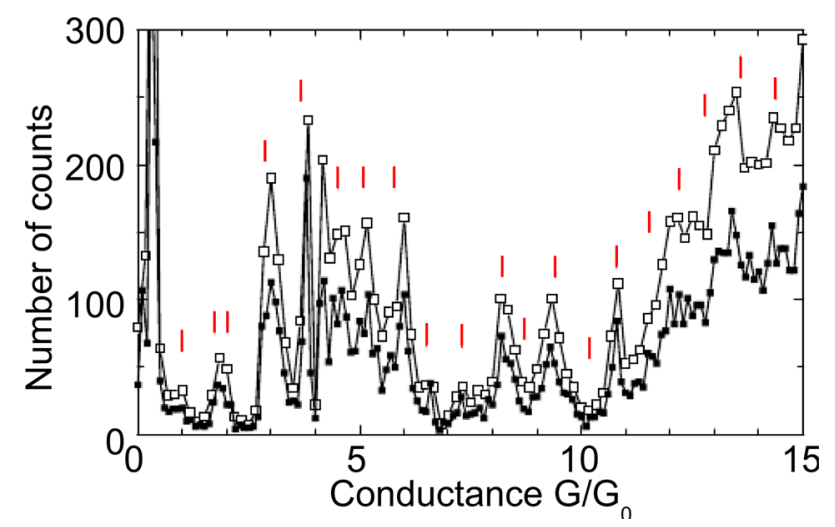

FIG. 20. Histogram generated from 5 Au samples. The red lines indicate the peaks as obtained from break-junction experiments for work-hardened $\mathrm{Au}$ contacts following Yanson et al. (2005). For the open symbols, a bin size of $(1 / 6) G_{0}$, and for the closed symbols, a bin size of $(1 / 10) G_{0}$ were used. Reprinted with permission from Hoffmann et al., Appl. Phys. Lett. 93, 043118 (2008). Copyright 2008 AIP Publishing, LLC. 


$$
G^{-1}=\frac{\rho}{2 R},
$$

where $\rho$ is the resistivity of the metal. The semiclassical formula for a ballistic nanowire circular cross-section yields

$$
G=G_{0}\left[\left(\frac{k_{F} R}{2}\right)^{2}-\frac{k_{F} R}{2}+\frac{1}{6}+\cdots\right]
$$

where $k_{F}$ is the Fermi wave-vector (Torres et al., 1994). The first term has been first found by Sharvin (Sharvin, 1965 and Kulik, 1992). Wexler has given a description of the transition between the two regimes (Wexler, 1966)

$$
G^{-1}=\frac{4}{3 \pi} \frac{\rho l}{R^{2}}+\gamma \frac{\rho}{2 R},
$$

where $l$ is the mean free path of the electrons and where $\gamma$ is a function well-described by (Nikolic and Allen, 1999)

$$
\gamma=\frac{1+0.83 l / R}{1+1.33 l / R}
$$

Such estimates for the diameter have been compared to SEM measurements and were found to give reasonable results (Gramich et al., 2012).

Predictions on the detailed structure are obtained both from tight-binding and from first-principles calculations. While the overall results are similar, differences occur in the details. First-principles calculations show that when one passes from a two atom-wide $\mathrm{Au}$ wire to a single-atomic $\mathrm{Au}$ wire, the conductance changes from two to one conductance quanta (Haekkinen et al., 2000). These calculations seem to suggest that for $\mathrm{Au}$, indeed the change in the number of channels is one channel for each atom. Other authors-using a combination of first principles and tight-binding methods-suggest that mostly metastable atomic configurations contribute to the conductance of a pulled Au nanowire, causing non-integer conductance plateaus, transitions, and slopes (Tavazza et al., 2011). It was found in one study that several atomic configurations contribute to the statistic weight of the peaks observed in histograms and that even for Au several channels contribute to each peak (Dreher et al., 2005), see Fig. 21. This is also supported by another group, who conclude that peaks in histograms are insufficient to characterize

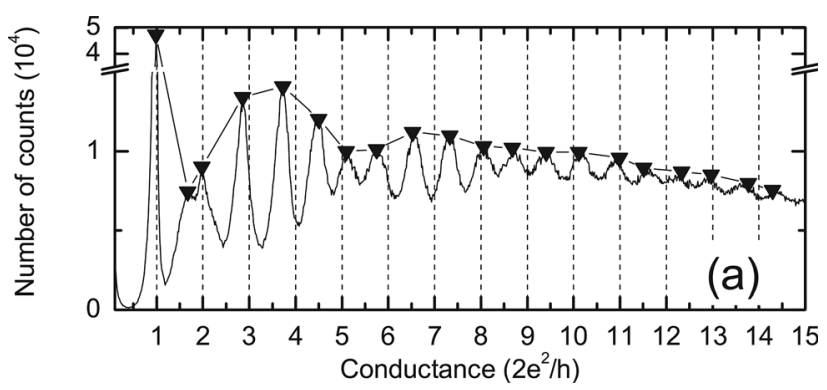

FIG. 21. Conductance histogram obtained at $6 \mathrm{~K}$ from roughly 20000 curves for work-hardened Au wires while breaking the contact. The peaks are regularly spaced and show a beating pattern that is analyzed and believed to result from the [001], [110], and [111] directions of Au. Reprinted with permission from Phys. Rev. Lett. 95, 256806 (2005). Copyright 2005 American Physical Society. the stability of atomic configurations (Armstrong et al., 2010). In this study, there is not a one-to-one relationship between a peak in a histogram and a particular atomic configuration. In summary, the question whether the conductance histogram peaks are caused by well-defined atomic configurations could depend on the parameters chosen for the model.

Another study, focusing on $\mathrm{Cu}$, stresses the importance of the electronic structure, and quantum size effects in particular, for the atomic configuration of the wire before the breaking point (Stepanyuk et al., 2004). For non-monovalent Al junctions, it was found that the conductance histogram indeed represents different atomic configurations (Hasmy et al., 2001).

\section{Electron transport across a gap}

\section{Vacuum tunneling}

The final result of thinning is to obtain a gap. Usually, to identify vacuum tunneling, the strong exponential distance dependence of the current is used. Another method of characterizing tunnel junctions relies on transition voltage spectroscopy and also needs a variation of the distance of the two electrodes (Trouwborst et al., 2011). However, not in all nanocontacts it is possible to vary the distance of the electrodes. This can make it difficult to identify the tunneling regime from other electronic transport regimes.

Often, it is believed that for tunneling to be a relevant electron transport mechanism, the overall resistance should be well above the quantum resistance, $12.9 \mathrm{k} \Omega$. However, if a notch is formed as discussed below in the section on structural properties (Sec. VE), it is possible that a large part of the notch constitutes a small gap where many tunneling contacts in parallel are active leading to a low total resistance.

We have observed a decrease for the resistance as a function of voltage together with nonlinearities for electromigration in vacuum, see Figs. 22 and 23 (Stöffler et al.,

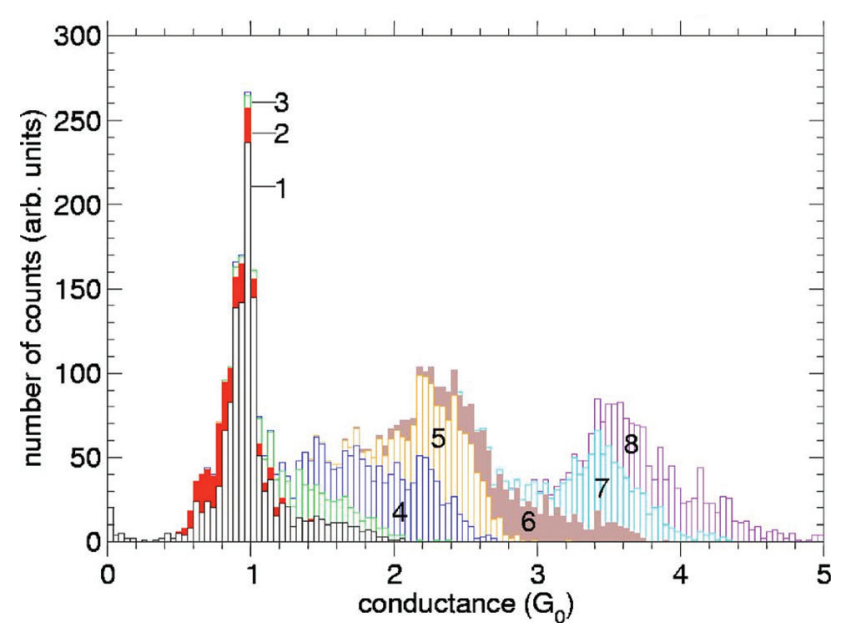

FIG. 22. Calculated conductance histogram using molecular dynamics simulations for $\mathrm{Au}$ in the [001] direction at $4.2 \mathrm{~K}$. Regions shaded in the same color represent a particular range of cross-sections of the nanocontact. It becomes clear that the cross-section is not the only parameter for determining the conductance of the atomic configuration, because different cross sections correspond to the same peak in the conductance histogram. Reprinted with permission from Dreher et al., Phys. Rev. B 72, 075435 (2005). Copyright 2005 American Physical Society. 

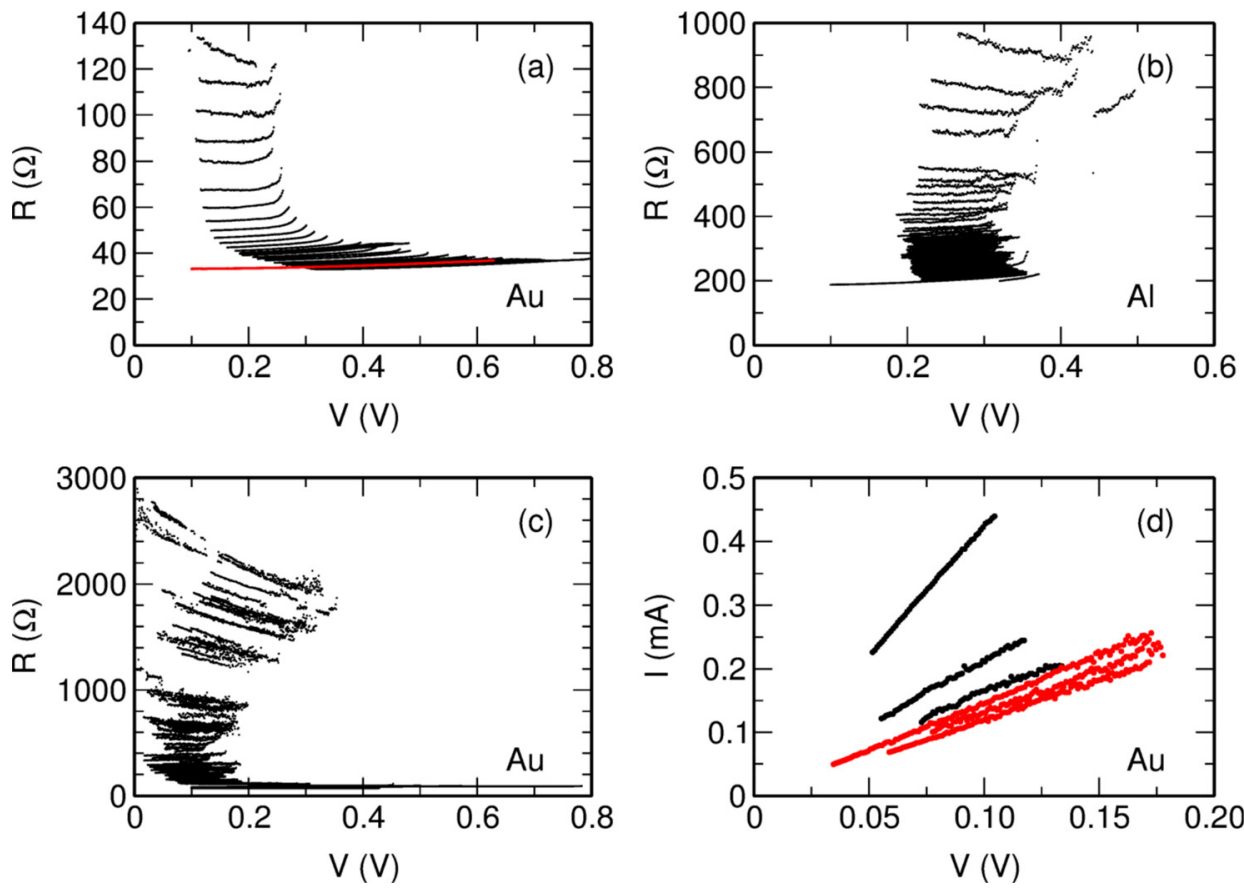

FIG. 23. (a), (b), and (c) Negative slopes in the resistance as a function of voltage for different metals such as $\mathrm{Au}, \mathrm{Al}$, and Pt. (d) $I(V)$ characteristics for the sample shown in (c) with data obtained in the regime with a positive slope (black) and in the regime with a negative slope (red). Reprinted with permission from Stöffler et al., Phys. Rev. B 90, 115406 (2014). Copyright 2014 American Physical Society.
2014). From the low total resistance in this regime (between 150 and $600 \Omega$ ), we assume that still part of the contact is connected. Both thermal hopping and tunneling could cause such a behavior. In order to understand the role of tunneling in this regime, we used a model where the tunneling distance changes as a function of voltage due to thermal expansion and/or electrostatic attraction. We modified the well-known tunneling model for two electrodes of differing materials (Brinkman et al., 1970 and Simmons, 1963) by making the distance a function of temperature for part of the contact due to thermal expansion or electrostatic attraction. Nonlinear current-voltage curves had also been found for Au nanocontacts and were attributed to a change in the distance of the electrodes that resulted in a thickening of the nanocontacts (Yoshida et al., 2005).

An important point is to distinguish tunneling and thermally activated hopping often observed in granular thin films. The $I(U)$ characteristics could be similar for thermally activated hopping compared to what is observed here. Often, in the intermediate temperature regime, thermal activation and tunneling occur simultaneously. For percolating tunneling granular films, conductance histograms show peaks as has been observed for tin clusters (Sattar et al., 2013). However, at the temperatures needed for electromigration, we expect granular films to break up and form larger grains due to heating. We then expect an increasing resistance as a function of voltage in contrast to the data shown in Fig. 22. Also, this view is not compatible with the slit formation observed in the contacts as described below in the section of direct structural characterization for thin film nanocontacts (Sec. V E).

\section{Transition to field emission}

For high bias voltages, in many geometries, a transition of ordinary tunneling to field emission has been observed (Young et al., 1971 and Trouwborst et al., 2011). Such a transition is most easily identified in a Fowler-Nordheim plot, where it causes a strong change of the characteristics, see Fig. 23(d). In the transition regime, Gundlach oscillations (Gundlach, 1966) resulting from resonant electronic states at the surface have been observed (Huisman et al., 2011 and Kolesnychenko et al., 1999). The energies of the resonant states can be studied, and the distance dependence of these oscillations can be used to determine the electrode distance.

In the field-emission regime, it is also possible to determine the distance of the electrodes using suitable models (Lambert et al., 2003). Field emission is often viewed as being detrimental for nanocontact formation, because it is often accompanied by material transport (Mayer et al., 1999; Wouters and Schubert, 2004; Tseng et al., 2005). However, such material transport can also be used to enhance the properties of the junction, a process often used for preparation of electron emitters in SEM. This activation process has also been used for the formation of nanocontacts (Kayashima et al., 2007 and Oon and Thong, 2004). Nanocontacts with conductances between 1 and $4 G_{0}$ can be formed by applying voltage pulses (Ittah et al., 2008).

\section{Coulomb blockade}

If a small conductive object, an island, occurs between two disconnected electrodes, two successive gaps are active. If the island is small, its capacity $C$ is small and the charging energy $e^{2} /(2 C)$ can become larger than the thermal energy $k T$. In this case, an additional electron cannot populate the island if it is already populated by one additional electron due to the Coulomb repulsion (Gorter, 1951; Beenakker, 1991; and Kastner, 1992). This has been observed with semiconductors (Meirav et al., 1990), and with metal islands (Fulton and Dolan, 1987 and Ralph and Buhrman, 1994) also using STM (Schönenberger et al., 1992 and Anselmetti et al., 1994). 
Controlled electromigration has been used as a technique to contact quantum dots made from other materials such as molecules (Park et al., 2002) or Si (Wolf et al., 2010): first, quantum dots were fabricated on a Si substrate, and then, the metal was evaporated on top and a gap of only a few $\mathrm{nm}$ was created by electromigration. The native oxide of Si was used as a tunneling barrier. In contrast to most studies discussed here, where electromigration is used to create a gap, electric field induced surface migration has been used to connect two separate gold leads (Anaya et al., 2003). For high resistances, Coulomb blockade was observed. In another study, electromigrated nanocontacts have been fully broken and were then subject to metal evaporation at room temperature, such that grains of $5-15 \mathrm{~nm}$ diameter were formed inside the gap (Bolotin et al., 2004).

In addition, such Coulomb Blockade effects are observed in nanoscale metallic contacts generated by electromigration without subsequent metal deposition (Houck et al., 2005; Sordan et al., 2005; and Noguchi et al., 2008), see Fig. 24. The origin of the Coulomb Blockade effects is here assumed to be metallic islands caused by the electromigration process. From the capacity of the islands, their size can be estimated and it is assumed that they consist of only a few atoms (Houck et al., 2005) or up to $3 \mathrm{~nm}$ in diameter (Sordan et al., 2005). Furthermore, level spectroscopy can be performed for the energetic levels of the confined electrons, if the contact can be addressed by a gate electrode. Level spectroscopy also shows signatures of vibrational modes of molecules and allows us to distinguish molecular and metallic grain effects (van der Zant et al., 2006). In Sec. IV C 1, it has been discussed how vacuum tunneling in addition to thermal expansion or electrostatic forces could lead to a negative differential resistance. Also, Coulomb blockade can lead to a negative differential resistance due to the blocking of the current. Negative differential resistances have been observed for Coulomb blockade in electromigrated nanocontacts (Noguchi et al., 2008).
The few-atom Au clusters present in the junction have been proposed as single-photon light sources due to their single quantum nature (Gonzalez et al., 2004b). Advantages are that they are usable at room temperature and that they can be electrically driven. Nanoscale metallic junctions made by controlled electromigration have also been integrated in radio frequency circuits with impedance matching (PueblaHellmann and Wallraff, 2011). This makes it possible to overcome limitations on the frequency to be used with these devices. Such limitations arise from the large resistances of the nanoscale metallic junctions.

The presence of such metallic grains in electromigrated contacts could limit their use for molecular electronics applications. In junctions made by self-breaking, no evidence of metal clusters was found, which may indicate that these are better suited for molecular electronics studies (O'Neill et al.,2007). On the other hand, localization of electronic states in the leads is also discussed as a possible origin of Coulomb blockade effects and a zero-bias anomaly (Bowman et al., 2004). We have found experimental evidence for electrically disconnected grains occurring in nanocontacts formed by electromigration by studying the local electrostatic potential using scanning force microscopy (Arnold et al., 2017).

\section{Reversing the current direction-cyclic behavior}

Due to the needs of the semiconductor industry for smaller functional elements, switches using nanoscale gaps between electrodes have been studied with great intensity. Often switches are controlled by a gate (Martin et al., 2009; Xie et al., 2004; Terabe et al., 2005; and Fuechsle et al., 2012) or by changing the electrode distance (Smith, 1995). Here, we discuss the behavior of the junction under the influence of an applied current or voltage without gate (Aono and Hasegawa, 2010). It has been known in scanning tunneling microscopy and field emission studies that atoms can be
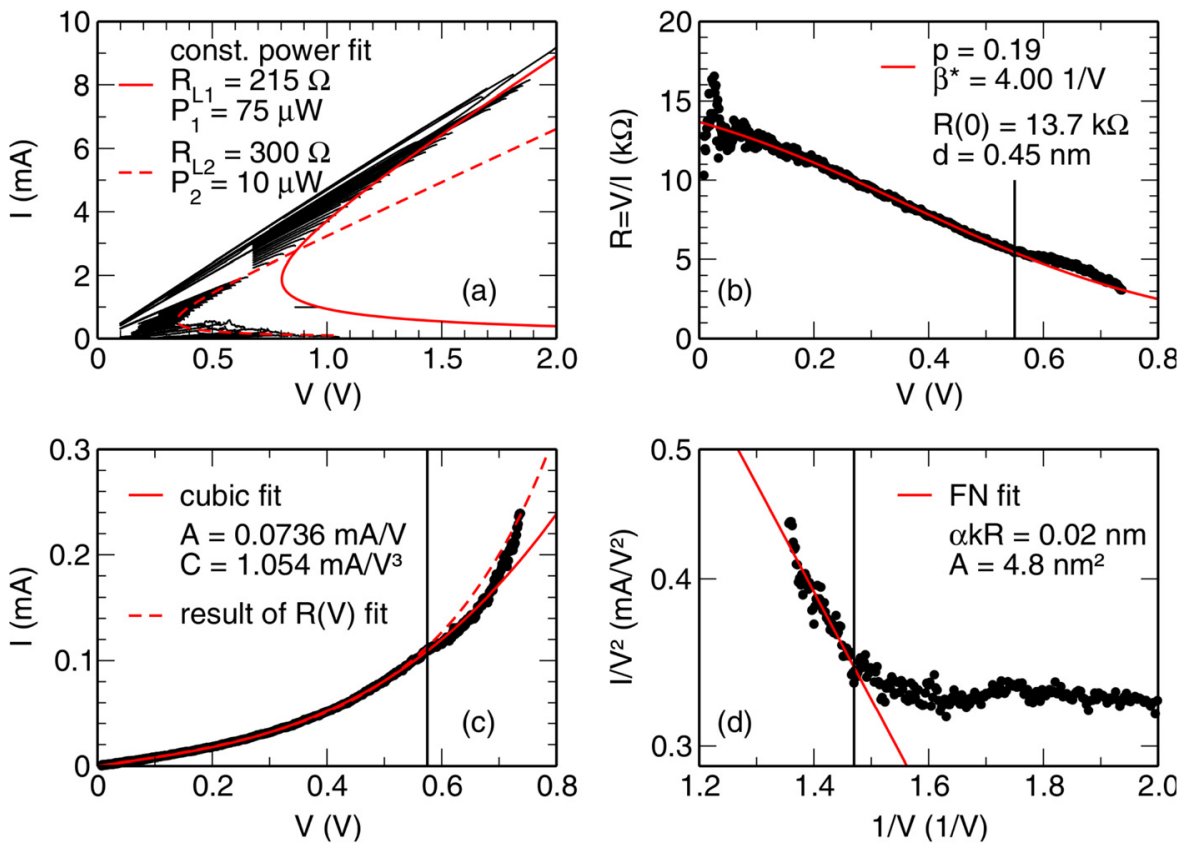

FIG. 24. (a) Overall electromigration characteristics for a Au sample for which both tunneling and field-emission are observed. (b) From a single cycle, it is difficult to tell the electronic transport mechanism. While for most of the electromigration processes the electronic characteristics are well-described by tunneling, as is shown by the fit in panel (c), the behavior of some samples shows Fowler-Nordheim characteristics, as shown in panel (d). Reprinted with permission from Stöffler et al., Phys. Rev. B 90, 115406 (2014). Copyright 2014 American Physical Society. 
emitted from tips (Mamin et al., 1990; Mayer et al., 1999; and Anaya et al., 2003). Field emission can also be used to tailor the gap size in nanocontacts (Kayashima et al., 2007). To form a controllable switch, it should in addition be possible to reverse the process. Such reversibles processes have been demonstrated (Naitoh et al., 2006; Strachan et al., 2008; Schirm et al., 2013; Lim et al., 2013; and Suga et al., 2016).

While at the macroscopic scale also an alternating current (AC) leads electromigration thinning, by careful tuning at the nanometer scale, reproducible switching can be obtained (Naitoh et al., 2006). While in one study, dramatic reversible changes of the resistance from $10^{8} \Omega$ to $10^{4} \Omega$ from the tunneling regime to a one or few-atom contact are observed for Au (Naitoh et al., 2006), changes on the order of a fraction the conductance quantum $G_{0}=2 e^{2} / h \approx 77 \mu \mathrm{S}$ are found in another study for $\mathrm{Al}$ (Schirm et al., 2013). Also, the methods for preparing the junction differ: in the first study, initially a gap of a few $\mathrm{nm}$ width is prepared (Naitoh et al., 2006), while in the second study, the junction is carefully thinned by stretching to yield a conductance a few conductance quanta (Schirm et al., 2013). The use of Al junctions allows us to analyze in addition the number of transmission channels of the junction due to a careful analysis of superconducting multiple Andreev reflections. During switching, the number of channels changes by one or remains the same. By molecular dynamics simulations where a tightbinding approach is used to describe the electronic structure, it is shown that atomic configurations containing only a few atoms at the junction describe the switching well (Schirm et al., 2013).

\section{E. Role of the chemical environment}

\section{Diffusive regime}

For many of the samples discussed here, the nanocontact is supported by the substrate and its surface is covered with adsorbates or at least in contact with air or vacuum. The effect of this environment (pressure of the residual gas, air humidity, etc.) on the nanoscale metallic conductor strongly depends on the size of the conductor and on the metal used. For relatively large conductors made of chemically inert metals such as $\mathrm{Au}$, it is generally accepted that the influence of the environment is small, simply because the influence of the surfaces on the electromigration process is small, in particular when grain boundary diffusion dominates the electromigration process.

For less inert $\mathrm{Cu}$ wires investigated under ambient conditions, strong effects of the environment are observed even for macroscopic wires (Hauser et al., 2016). While for $\mathrm{Au}$, a ramp to higher voltage is succeeded by the start of the hyperbolic curve that we expect for electromigration at constant power dissipated at the forming contact [Eq. (22)], see Figs. 14 and 25, for example, for $\mathrm{Cu}$ a clearly distinct shape is observed. A concave part of the curve formed by the endpoints is typically detected at the onset of electromigration, see Fig. 26. We associate this part of the curve with oxidation of the $\mathrm{Cu}$. Much more power is needed for the first electromigration cycles compared to later cycles. Successive

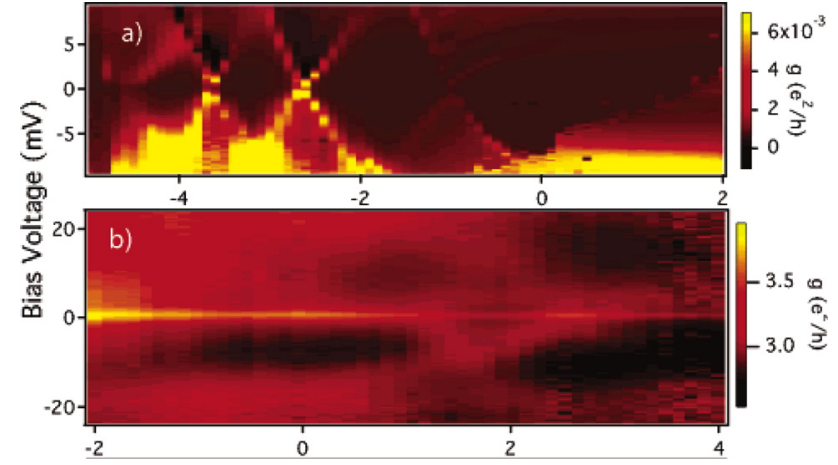

FIG. 25. Differential conductance measured at $250 \mathrm{mK}$ (a) Coulomb blockade and (b) superposition of a broad Coulomb diamond centered around 1.8 $\mathrm{V}$ and the Kondo-effect due to hybridization of spin-polarized states on the island with conduction electrons. Reprinted with permission from Houck et al., Nano Lett. 5, 1685 (2005). Copyright 2005 American Chemical Society.

oxidation during electromigration leads to a coating of the wire surface. It is well-known that a coating of a wire can counterbalance mechanical stress built up by the diffusion of atoms during the electromigration process and thus enhances resistivity to electromigration by mechanically stabilizing the wire (Hau-Riege, 2004). This could be responsible for the increase in power needed in the first part of the electromigration thinning cycles. Also, for coated $\mathrm{Cu}$ interconnects without sealing, a detrimental influence of moisture has been found (Cheng et al., 2010). It was concluded that sealing is essential for protecting the $\mathrm{Cu}$ interconnects. The effect of moisture depends strongly on the microstructure of the sealing (Cheng et al., 2014). Dense dielectrics are more beneficial than porous materials.

This is in agreement with additional experimental results: The value of $Z_{S}^{*} D_{S}$ has been determined experimentally for $\mathrm{Cu}$ to be $3.35 \times 10^{-12} \mathrm{~m}^{2} / \mathrm{s}$ (Choi et al., 2007), six orders of magnitude smaller than previously reported values (Hu et al., 1999), meaning that electromigration is much slower on clean $\mathrm{Cu}$ surfaces than on oxidized ones. The

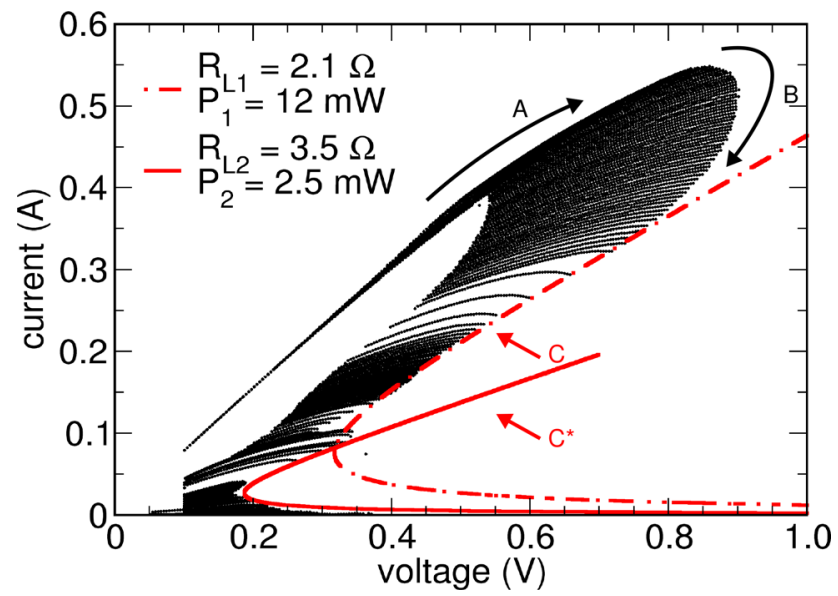

FIG. 26. Initial electromigration stages for a $\mathrm{Cu}$ wire under ambient conditions. A concave part of the curve denoted by the letter B is observed. This part cannot be described with the hyperbolic curves that describe the other parts of the curve well. This concave part is related to oxidation of $\mathrm{Cu}$. Reprinted with permission from Hauser et al., Appl. Phys. A 122, 1068 (2016). Copyright 2016 Springer International Publishing AG. 
differences between this study and the previous ones were explained by different surface conditions, in particular, the presence or absence of oxidation.

\section{Few atom contacts}

In metallic nanocontacts where the current runs over only a few atoms, it is clear that changing the nature of just one atom in the region of the contact itself has a profound effect on the conductance properties of the nanocontact, changing qualitatively the nature of electron conduction, as has been shown, for example, by calculations (Mehrez et al., 2002). From the experimental point of view, it is clear that even seemingly inert surfaces such as Au are subject to changes due to air and/or moisture as has been shown by scanning tunneling microscopy studies (Roberts et al., 1991).

Indeed, even a hydrogen molecule, present in the area of the metallic $(\mathrm{Pt})$ contact, could be viewed as a molecular electronics device (Smit et al., 2002; Csonka et al., 2003; and Jelínek et al., 2006). For Au contacts investigated by the electromigration method, mainly oxygen had a clear effect, leading to uncontrolled behavior and early breaking of the forming nanocontact at room temperature (Stöffler et al., 2014). On the other hand, at liquid-helium temperatures the formation of atomic chains in $\mathrm{Au}$ and $\mathrm{Ag}$ was enhanced in the presence of oxygen (Thijssen et al., 2006). For Ag, atomic chains were formed only in the presence of oxygen. This finding was compared to the formation of oxygeninduced reconstructions on $\mathrm{Ag}$ [110] surfaces. Calculations, where the influence of $\mathrm{O}_{2}$ and $\mathrm{H}_{2} \mathrm{O}$ impurities on the stability and conductance of Au nanowires was investigated, confirmed that oxygen enhances the formation of atomic chains in Au (Barzilai et al., 2013). It was found that in particular oxygen enhances the stability of Au nanowires, but on the other hand, this impurity was expelled from the monoatomic chain during pulling and had little influence on the conductance. Another study investigated trapping of a water molecule between two electrodes formed by depositing $\mathrm{Au}$ between two free-standing leads until an electron tunneling connection is formed (Korotkov et al., 2003). It was found that water trapped in the tunneling barrier increases the tunnel barrier height.

Oxygen dramatically influences the properties of $\mathrm{Al}$ nanocontacts, making them more brittle and shortening the maximum displacement before breakage (Jelínek et al., 2005). The effect is small for hydrogen and intermediate for carbon. For $\mathrm{Fe}, \mathrm{Co}$, and Ni nanocontacts investigated by the break-junction method, the number of peaks in histograms depend on the residual gas of the environment (Untiedt et al., 2004). For Ni electrodes, exposure to atmosphere was detrimental to the anode side of the electromigration setup (Yoshida et al., 2009).

\section{Consequences for studies of nanoscale metallic junctions}

In view of these results, questions about the fabrication process are raised. Electromigration fabrication of nanocontacts needs a small metallic bridge to start with. In many

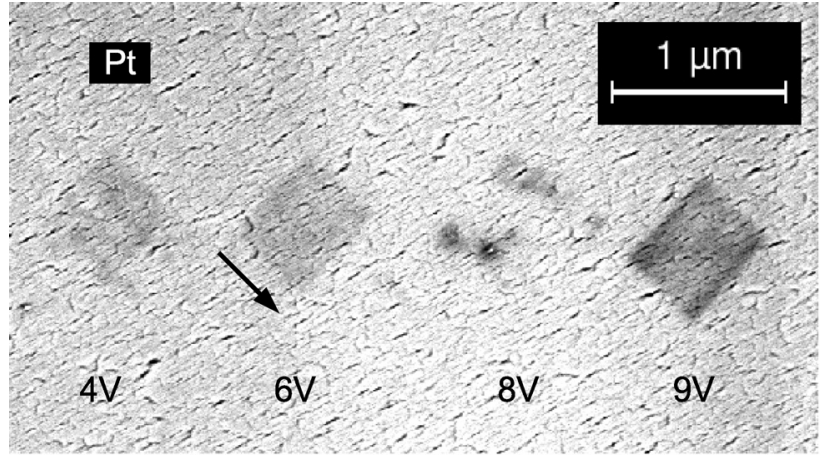

FIG. 27. SEM image of deposits on a Pt surface after electron-beam lithography and subsequent STM imaging. Under each deposit, the voltage used during STM imaging is shown. With increasing voltage, the intensity of the deposits increases. Reprinted with permission from Stöffler et al., Nanoscale 3, 3391 (2011). Copyright 2011 The Royal Society of Chemistry.

cases, these metallic bridges are fabricated by electron beam lithography as discussed above. It involves the application of resist and solvents to the sample. It has been previously known from surface science studies that even intense rinsing of surfaces does not always lead to clean surfaces at the atomic scale, and therefore, it is largely accepted to perform extensive cleaning steps in ultra-high vacuum before a surface is considered clean, for an overview, see Musket $e t$ al. (1982). For electromigrated structures, it has also been found that residues induced by the electron-beam lithography process can spoil the cleanliness of the surface (Stöffler et al., 2011). Using a large STM tunneling voltage (up to $9 \mathrm{~V}$ ), impurities can be immobilized at the areas scanned with the tip and appear as elevated islands in STM images, Fig. 27.

The results from this section show that it is important to control the environment in which the nanocontact is formed precisely. One possibility is to avoid electron beam lithography usually employing organic resists and instead use mask evaporation methods already discussed in the section on fabrication (Sec. II).

\section{DIRECT STRUCTURAL CHARACTERIZATION OF METALLIC NANOCONTACTS}

In contrast to statistical considerations obtained from calculations and from measuring the overall properties of a large number of samples, imaging methods aim at studying individual cases and try to relate particular microstructures of particular samples to particular phenomena observed in these cases. Since each sample is an individual case, the results differ from sample to sample.

Four different sample geometries and microstructures are considered here with a focus on controlled electromigration: Single-crystalline nanowires, supported thin films, and freestanding thin films of pure metals are discussed.

\section{A. Imaging methods and their limitations concerning imaging of nanocontacts}

In the following, images obtained from different experimental techniques are shown in conjunction with each other. Since the different imaging techniques are based on 
fundamentally different physics, it is important to interpret the images according to the imaging methods used.

Electrons have a limited escape-depth and are therefore well-suited to characterize samples at the nanoscale (Stierle et al., 2012). In transmission electron microscopy, a flow of electrons of high energy is used for investigating a sample. The sample must be electron-transparent. For imaging nanocontacts, this is often reached by using a thin film contact on electron-transparent substrate, mainly $\mathrm{Si}_{3+x} \mathrm{~N}_{4-x}$, or by using a free-standing thin metallic layer. To operate a TEM, vacuum is needed. Often through the strong bombardment of the surfaces inside the TEM apparatus and the generation of the electron beam, carbon-rich components of the residual gas are produced. These can be redeposited on surfaces in the course of imaging and influence the results. The TEM uses transmission through the sample and can therefore give information of the three-dimensional body of the sample. Multiple scattering presents an additional complication for the interpretation of high-resolution TEM images. A TEM is rarely combined with low temperatures by cooling the sample with liquid nitrogen. TEM observation of electromigration in thin films has a long tradition (Blech and Meieran, 1967).

In the section on fabrication of metallic nanocontacts, it has been briefly described how the electron beam can also be used to alter the structure of the metallic layer and create a nanocontact. If transmission electron microscopy is used for imaging, care must be taken to find a regime where the influence of the electron beam and the heat deposited on the sample's structure is negligible. Indeed, electromigration by the influence of the electron beam has been observed (Ichinokawa et al., 1993 and Stahlmecke and Dumpich, 2007a).

Another class of imaging methods applied successfully for metallic nanocontacts is X-ray investigation. Mostly, intense X-ray radiation from synchrotrons has been used to study electromigration (Zschech et al., 2009; Budiman et al., 2006; Chen et al., 2008a; and Takahashi et al., 2009). Rarely, also X-rays in electron microscopes have obtained sufficient resolution to study electromigration in thick samples (Frigo et al., 2002). One technique is X-ray tomography where the absorption of radiation is studied locally (Zschech et al., 2009). Another technique is X-ray microdiffraction where diffraction spots resulting from a small sample volume are analyzed (Budiman et al., 2006 and Chen et al., 2008a). In this way, the crystallographic orientation of grains can be studied. Using coherent X-ray diffraction techniques, the speckles generated from $\mathrm{Cu}$ lines have been investigated and considerations on the real-space structure were obtained from phase-retrieval algorithms (Takahashi et al., 2009).

Scanning force microscopy can be used in a variety of environments, such as liquids, vacuum, low or high temperatures. In scanning force microscopy, the tip-sample interaction, or its derivative, the force, is obtained by measuring the bending of a microscopic cantilever-spring the tip is attached to (Morita et al., 2002; Garcia and Perez, 2002; and Giessibl, 2003). This force comprises short-range and long-range components (Guggisberg et al., 2000). The short-range component originates both from chemical bond formation and from electrostatic and van-der-Waals interactions (Lantz et al., 2001; Hoffmann et al., 2004, 2003; Cross et al., 1998; and Hoffmann, 2009). In scanning force microscopy, often dynamic operating modes are used (Albrecht et al., 1991). Highest resolution is obtained in modes where hard contact between the tip and the sample is avoided, because hard contact could damage the tip apex (Kitamura and Iwatsuki, 1995 and Giessibl, 1995). The tip is usually assumed to have a radius below $2 \mathrm{~nm}$ and a conical shape at the back side of its front sphere (Hudlet et al., 1999 and Argento and French, 1996). It has been debated whether three-dimensional objects can be imaged with high resolution due to the shape of the tip. The atomic or molecular structure of steps and even the facets of large molecular islands can be imaged successfully with high resolution using scanning probe microscopy (SPM) methods (Kunstmann et al., 2005; Pérez León et al., 2016).

\section{B. Elementary processes}

\section{Atomic hops and surface diffusion}

At the basis of every diffusion or electromigration process are atomic hops. The nature of these atomic hops, e.g., the crystallographic direction of the hops and whether they occur near defects, strongly influences the structural changes in surface diffusion and electromigration. Atomic hops have been studied extensively both experimentally and theoretically. We have already mentioned that Black's law [Eq. (4)] offers the possibility of determining the local binding energies if one is able to favor a certain diffusion path during electromigration. Here, we focus on ways to obtain information on individual atomic hops. This has become possible using scanning probe techniques (Hoffmann et al., 2008; Paul et al., 2013; and Landman and Luedtke, 1991).

Atom manipulation has been performed in both STM and SFM techniques. STM is well established as an engineering tool for atom manipulation (Eigler and Schweizer, 1990; Stroscio and Eigler, 1991; and Fishlock et al., 2000) while for SFM atom manipulation has been demonstrated more recently (Sugimoto et al., 2005). SFM allows us to measure the force during atom manipulation, see Fig. 28 (Ternes et al., 2008). This is related to electromigration,

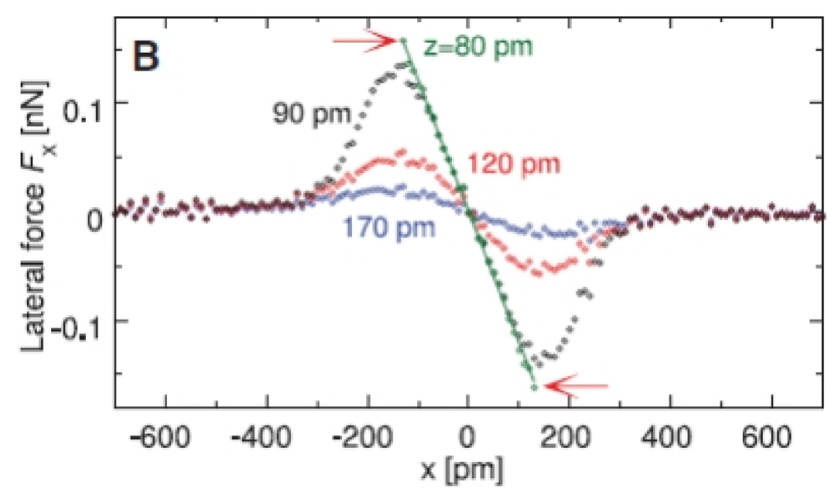

FIG. 28. Force to move a single $\mathrm{CO}$ molecule on $\mathrm{Cu}$. $\mathrm{Up}$ to $0.2 \mathrm{nN}$ are needed to move these molecules on this surface. Reprinted with permission from Ternes et al., Science 319, 1066 (2008). Copyright 2008 American Association for the Advancement of Science. 
because it gives an estimate of electromigration forces. Both the forces needed for atom manipulation and the forces needed for electromigration allow overcoming similar energy barriers and are therefore expected to be of similar magnitude. The electromigration force has been extracted from the dynamical properties of the $\mathrm{Ag}$ islands (Tao et al., 2010). A value of $10^{-14} \mathrm{~N}$ was obtained for an island edge site, considerably smaller than the value obtained for the manipulation of a single $\mathrm{CO}$ molecule on $\mathrm{Cu}$ in Fig. 28.

Electromigration-induced atom manipulation of single Ni by STM atoms was studied on a Ni(111) surface (Braun et al., 2007), Fig. 29. The movement of the atoms results from a balance between attractive chemical forces pulling the $\mathrm{Ni}$ atoms towards the tip and pushing related to the tunneling current, possibly enhanced by resonant tunneling. The repulsive interaction can be inferred over a distance of up to $6 \mathrm{~nm}$. The dominant role of the inhomogeneous electric field, i.e., the direct force, and of electromigration in STM atom manipulation has become clear (Stroscio et al., 1992; Tsong, 1991; and Braun et al., 2007).

Surface diffusion has been studied both experimentally and theoretically. For recent reviews, see Barth (2000), Rosei (2004), and Misbah et al. (2010) and in particular (Brune, 1998), where the focus is laid on metals on metal surfaces. Here, we will focus on some particular aspects important for electromigration.

One issue is the role of gaseous impurities and air humidity for seemingly inert noble metals. The diffusion of
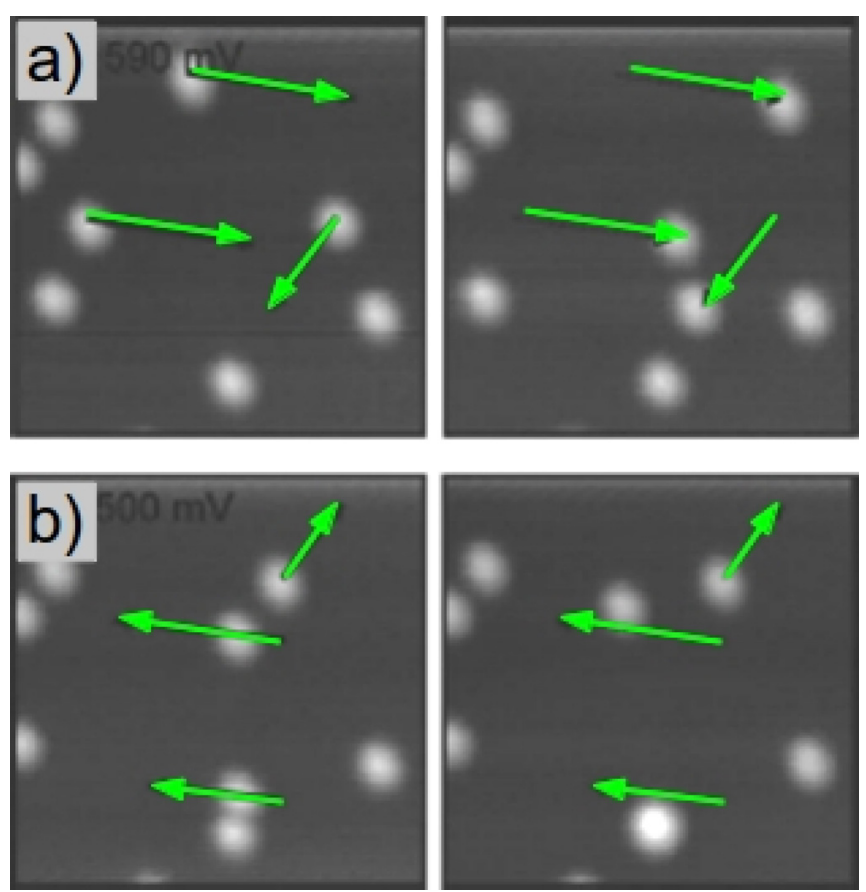

FIG. 29. Pairs of STM images $\left(0.8 \times 0.8 \mathrm{~nm}^{2}\right)$ with attempts to move single $\mathrm{Au}$ atoms laterally on a $\mathrm{Ni}(111)$ surface using electromigration. The left images of (a) and (b) show the initial state while the right images show the final state. The green arrows indicate the tip paths at closer tip-sample distance. (a) For positive voltages, such as $+590 \mathrm{mV}$ used for these manipulation attempts the atoms follow the STM tip. (b) For negative voltages, such as $-500 \mathrm{mV}$ used for these manipulation attempts the atom tends to move away from the STM tip. Reproduced with permission from Appl. Phys. Lett. 90, 023118 (2007). Copyright 2007 AIP Publishing.
$\mathrm{Au}$ on $\mathrm{Au}$ wires was studied in an inert $\mathrm{Ar}$ atmosphere (Roberts et al., 1991). A strong surface mobility was found even at room temperature, while the surface was found to be stable in air. Upon injecting traces of water or air into the chamber, the surface morphology changed. Studies such as this one make clear that a very good vacuum (ultra-high vacuum) is essential for understanding diffusion processes on surfaces.

Another point is the role of collective motion of atoms as has been observed for the $\mathrm{Au}(100)$ surface (Labayen et al., 2003), see also Fig. 30. A collective motion perpendicular as well as along five-atom wide strings on the $\mathrm{Au}(100)$ surface has been observed using video-rate STM. For the diffusion of $\mathrm{CO}$ molecules on $\mathrm{Cu}$, it has been observed that dimers and chains diffuse substantially faster than single molecules (Briner et al., 1997).

\section{Surface electromigration}

Surface electromigration of $\mathrm{Au}$ and $\mathrm{Ag}$ thin films grown on insulators or semiconductors has been studied early on with STM (Reiss et al., 1992) and SEM (Ichinokawa et al., 1993) measurements. Typically, additionally to the tunneling current used for imaging, another current was passed through the sample while imaging employing a modified STM (Reiss et al., 1992; and Maldonado et al., 2011).

In SEM studies of various metal films, directional diffusion was found (Ichinokawa et al., 1993). Usually, directional diffusion of atoms from the anode to the cathode is observed (Besold et al., 1994). As a consequence of atomic directional diffusion, also islands diffuse by step edge diffusion. The diffusion of single $\mathrm{Ag}$ islands under the influence of a current in the direction of the electron flow has been studied using STM (Tao et al., 2010). At the step edge, the momentum transfer from the current to the atoms confined at the step is larger than on the free surface (Bondarchuk et al., 2007). Electromigration leads to fluctuations of the step. The correlations of these fluctuations as a function of temporal delay are found to agree with a recent model. These fluctuations are a significant contribution to the fluctuations of the electronic properties of nanostructures.

Electromigration of surface islands has also been studied theoretically using both continuum (Kuhn et al., 2005 and Pierre-Louis and Einstein, 2000) and atomistic approaches (Mehl et al., 2000). Periphery diffusion, i.e., diffusion along the step edge of an island, diffusion on a terrace, and twodimensional evaporation-condensation, i.e., attachment and detachment from a step edge, have been considered (Pierre-Louis and Einstein, 2000). Periphery diffusion is the dominant process (Mehl et al., 2000 and Pierre-Louis and Einstein, 2000). An effect similar to the Bernoulli effect is active, where the pressure variation in the direction perpendicular to the flow is proportional to the kinetic energy in this flow (Pierre-Louis and Einstein, 2000). This leads to elongation of the surface island. In addition, splitting of islands has been observed. However, the system can also be driven to the limit where terrace diffusion or evaporation of single molecules from islands to a two-dimensional surface gas phase and condensation on the islands are the dominant 

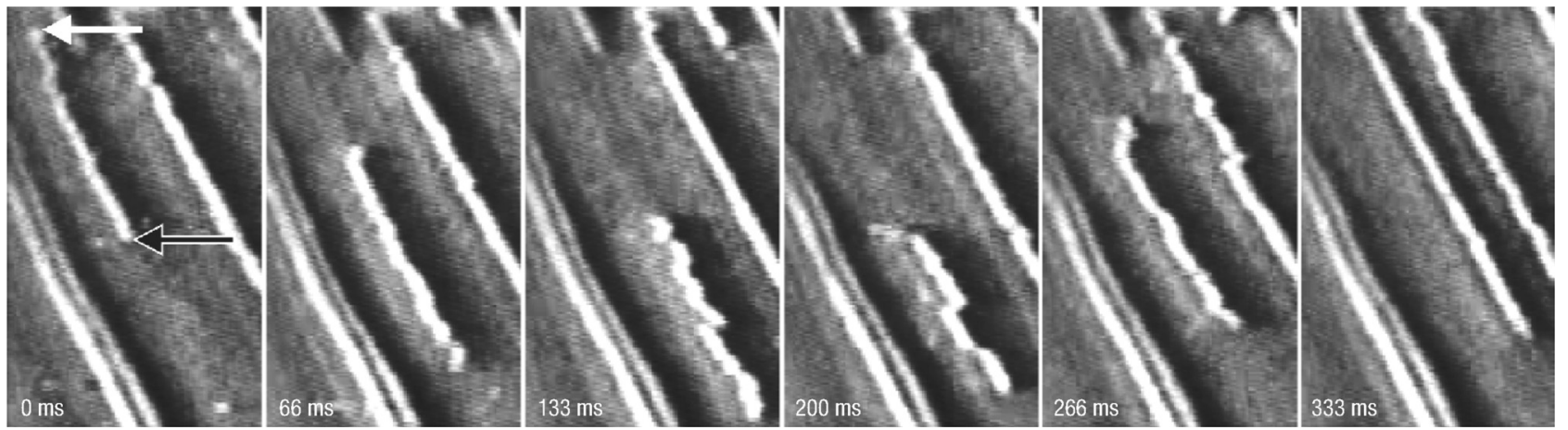

FIG. 30. Collective motion of Au stripes on a Au [001] surface as observed with STM. Reprinted with permission from Labayen et al., Nat. Mater. 2, 783 (2003). Copyright 2003 McMillian Publishers Ltd.

processes. In this limit, slit formation either in the direction parallel or perpendicular to the electromigration forces is observed in the simulations.

A $200-\mathrm{nm}$ thick $\mathrm{Ag}$ film was observed to develop new grains ("nodules") as a result of electromigration (Levine et al. 1993). Similarly, a Au film was also observed to develop clusters due to the application of an electric field (Mendez et al., 1996). Also, the appearance of additional steps due to electromigration on $\mathrm{Cu}$ grain surfaces has been observed using TEM (Chen et al., 2007). These steps appear on [111] surfaces and the atoms composing the step move towards one of the [110] directions that is part of this plane.

As a consequence of prolonged electromigration, coalescence of islands and faceting were observed (Besold et al., 1994). In calculations when crystal anisotropy is added, the formation of stable facets is observed (Kuhn et al., 2005). The shape of the islands can then in addition show oscillatory dynamic behavior and a zigzag structure. From the atomistic calculations, it is found that for periphery diffusion to occur, atoms are detached from corners (Mehl et al., 2000).

The anisotropy of void motion has also been observed experimentally (Latz et al., 2014). It was found for monolayer islands and voids on $\mathrm{Ag}$ surfaces that the motion of voids does not necessarily follow the direction of the current flow but can form an angle with this direction if the current does not flow in a high-symmetry direction of the surface.

\section{Single-crystalline nanowires}

Electromigration is strongly influenced by structural defects. In bulk samples, usually grain boundary diffusion or other defect-mediated diffusion dominates electromigration. In this view, one could ask the question what happens if such defects are absent. In addition, it is known that during the last stages of the electromigration process, a single grain separated by grain boundaries is left (Strachan et al., 2008). Studies of electromigration of single crystals allow us to focus on the processes that occur in this final stage.

In early studies, wires etched from bulk single crystals had been investigated (Shingubara et al., 1991 and Joo and Thompson, 1997). It was found that they resisted more strongly to electromigration, mostly because void formation parallel to the line (interconnect) was observed (Shingubara et al., 1991). Also, forming voids showed signs of faceting (Joo and Thompson, 1997). Such samples showed a large number of surface defects which influenced the results.

Sample preparation in recent studies of single-crystalline nanowires is very advanced and based on the fabrication of thin-film single crystals. Electromigration was found to depend strongly on the crystallographic direction (Sindermann et al., 2013). In another study, single-crystalline Ag wires were fabricated by self-assembly on vicinal Si surfaces in ultra-high vacuum (Stahlmecke et al., 2006). In the initial growth stages, faceting of the Si surface was observed. If one continues the Ag deposition after completing the faceting, the excess amount of $\mathrm{Ag}$ experiences directional diffusion due to the alignment of the steps on the vicinal surface. This leads to the growth of nanowires in addition to compact islands. A long wire is searched for and contacted by electron-beam lithography. The electromigration process and electrical measurements are performed in situ in the SEM. In another study, a four-probe STM equipped with an SEM was used to contact a similarly grown Ag nanowire in situ in the ultra-high vacuum chamber without ever exposing the sample to air (Kaspers et al., 2009), see Fig. 31.

For single-crystalline nanowires, the defect concentration can be sufficiently low to suppress electromigration along the wire (Stahlmecke et al., 2006). Electromigration then occurs at the contacts of the wire rather than along the wire itself. Two processes could be responsible for this behavior: 1. At the contact between the wire and the macroscopic contacts, there could be an excess amount of defects, e.g., due to impurities incorporated into the material during
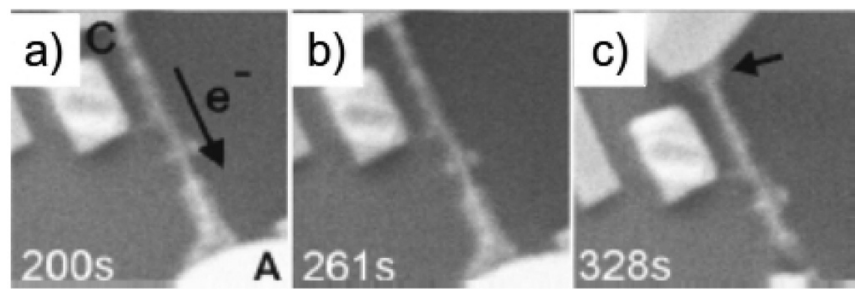

FIG. 31. Evolution of a Ag nanowire under the influence of a current of 80 $\mathrm{mA}$ flowing in the direction indicated in image (a). These images are taken from a movie at different times. "A" and "C" denote the anode and the cathode. The arrow in (c) highlights the formation of an extrusion. Reprinted with permission from Kaspers et al., J. Phys.: Condens. Matter 21, 265601 (2009). Copyright 2009 Institute of Physics Publishing. 
the fabrication process. As a consequence, the resistivity could be large in the overlap region between the nanowire and its large contacts. 2. The flow of atoms along the wire shows a discontinuity at the joint to the contact, because the width of the arrangement changes. This could cause a divergence in the flux of vacancies at the position of the joint and as a consequence electromigration first starts at the joint. It would be interesting to know whether the nanocrystal length was smaller than the Blech length.

In order to avoid electromigration at the joint, prior to electromigration a defect was introduced into the singlecrystalline wire by scratching it using an STM tip (Kaspers et al., 2009). With this method, electromigration is indeed induced to start at the location of the defect. The mass transport of Ag opposes the direction usually expected: it opposes the flow of electrons instead of following it (Stahlmecke et al., 2006 and Kaspers et al., 2009). The authors suggest that the direct force is in this case stronger than the wind force in contrast to what is usually observed for metals. A similar behavior is rare for pure metals but has been observed in liquid metal nanobridges (Zhao and Zhu, 2010). This point needs further investigation.

Slit formation in single-crystalline nanowires has also been studied by simulations taking into account surface diffusion, surface electromigration, and current-crowding (Mahadevan and Bradley, 1999). Slits similar in shape to experimental observations extend at a small angle with respect to the direction opposite to the electron flow.

\section{Grain boundary electromigration}

In order to investigate grain boundary electromigration in detail, a method to prepare nanowires of single crystals with the exception of only one grain boundary has been proposed and its feasibility demonstrated (Sindermann et al., 2011). First, Ag is deposited on an oxidized Si surface and a suitable polycrystalline island with large single-crystalline areas is identified. Then, the sample is moved to a focused ion beam device and a nanowire with contacts is made. Voids nucleate in the wire and move to the cathode under the influence of the windforce. Reversing the current direction allows us to reverse the direction of movement of the voids. The voids even pass the grain boundary.
Electromigration in polycrystalline solids has also been observed using high-resolution TEM (Chen et al., 2008b), Fig. 32. It was found that introducing twin boundaries (TB) has a profound retarding impact on grain boundary electromigration.

\section{E. Substrate-supported thin films}

\section{Fabrication details of thin-film wires with a constriction}

Supported polycrystalline metal thin films are the type of samples most frequently studied using controlled electromigration. In recent experiments, mostly $\mathrm{Au}$ thin films, but sometimes also Ag (Gardinowski et al., 2006 and Masuda and Kizuka, 2014), Pd (Arzubiaga et al., 2013), Pt (Suga et al., 2011; Gao et al., 2011; and Strigl et al., 2015), Pb (Luo and Yao, 2009), Cu (Hauser et al., 2016 and Kizuka and Aoki, 2009), Ni (Yoshida et al., 2010), and other metals have been used. The microcrystalline properties of the metal films strongly depend on the substrate, precise deposition method and parameters, and the film thickness. In experiments on $\mathrm{Si}_{3+x} \mathrm{~N}_{4-x}$, the $\mathrm{Au}$ grains have sizes between 10 and $100 \mathrm{~nm}$ (Strachan et al., 2006, 2008; and Heersche et al., 2007), similar to oxidized bulk $\mathrm{Si}$, where the typical grain size is about $30 \mathrm{~nm}$ (Stöffler et al., 2012). The contacts are usually produced by electron-beam lithography in a bow-tie or similar structure with a weak point at which breakage is expected. In some cases, instead of the PMMA masks usually used in electron beam lithography, solid masks have been used to create the structure (Stöffler et al., 2012). Often, from the half shadow of the mask during evaporation, unconnected islands appear next to the wire structure (Strachan et al., 2006 and Stöffler et al., 2012).

\section{Overall thinning process}

In the electromigration of such thin-film contacts, the dominant process is the formation of a notch of several to several tens of nanometers width (Blech and Meieran, 1967; Strachan et al., 2006, 2008; Heersche et al., 2007; and Stöffler et al., 2012), Fig. 33. This notch appears near the cathode for long wires, and at the weak point for bow-tie structures (Cho et al., 2011). As expected, the material is
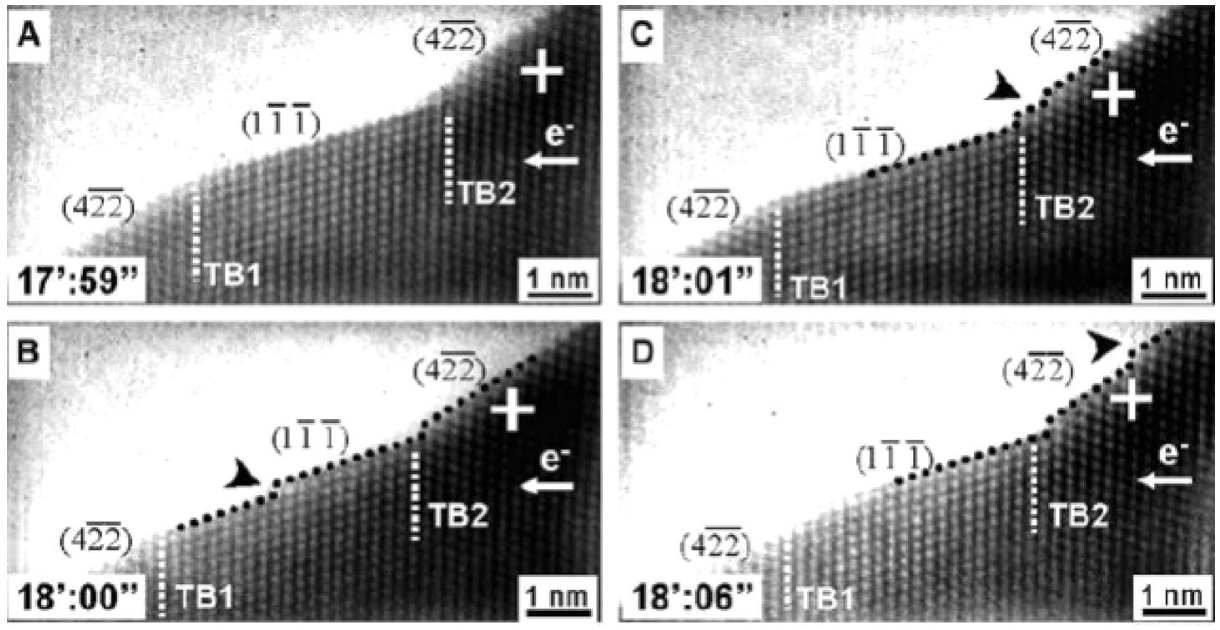

FIG. 32. Evolution of a (110)-oriented grain in a polycrystalline $\mathrm{Cu}$ wire under the influence of an electric current with time, indicated in the images, as observed with TEM. The arrows indicate some atomic steps, and also twin boundaries (TB) are indicated. The cross indicates the same position in each frame as a help to the reader. It was found by analyzing such images quantitatively that twin boundaries significantly retard electromigration. Reprinted with permission from Chen et al., Science 321, 1066 (2008b). Copyright 2008 American Association for the Advancement of Science. 


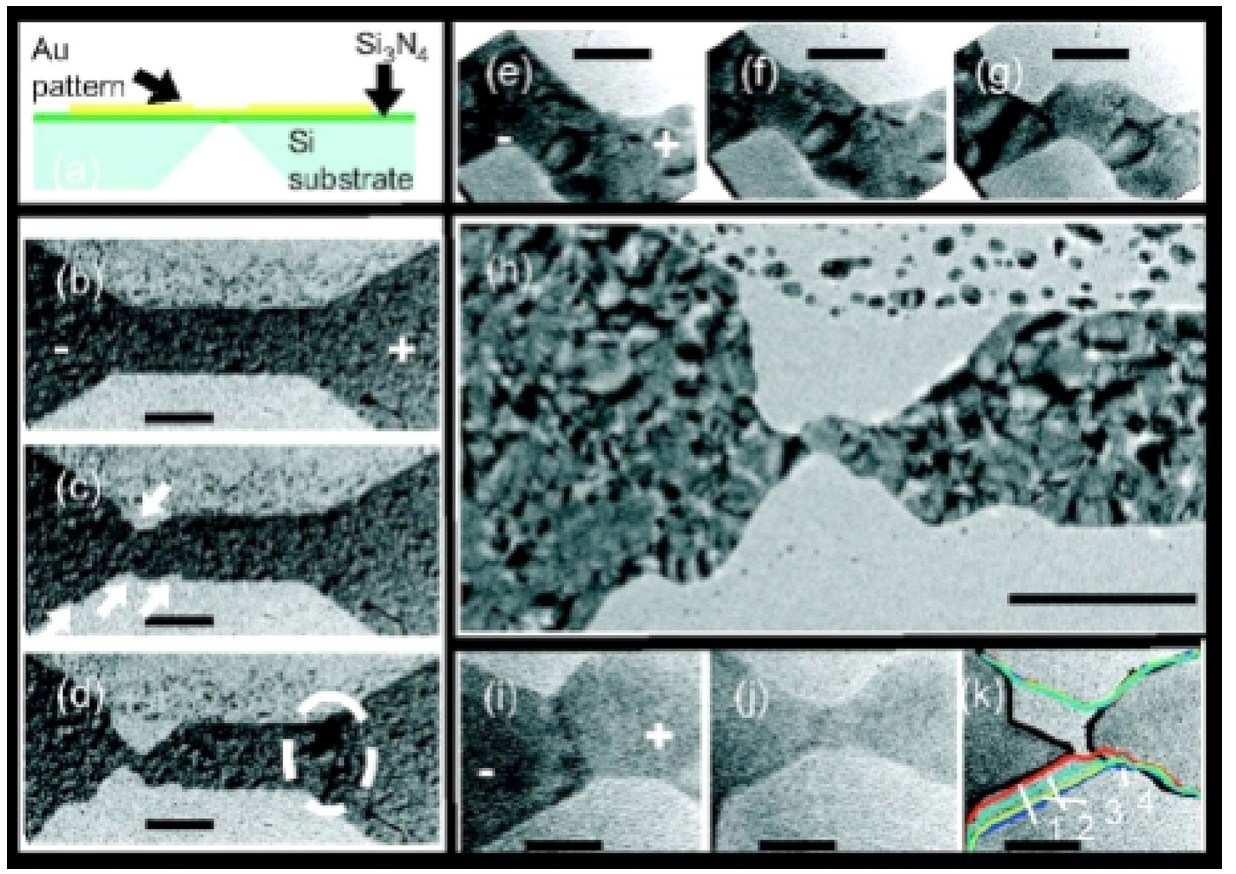

FIG. 33. (a) Schematic cross-section of the sample geometry. (b) Initial device before passive breaking by applying a voltage ramp. (c)-(k) Further development of the sample structure during breaking. First, voids develop at the cathode side (c) and hillocks at the anode side [see white circle in (d)]. The arrows indicate grain boundaries. In (b)-(d), the scalebar corresponds to $200 \mathrm{~nm}$. (e)-(g) Zoom in near the point where breaking takes place. The wire develops until the current runs over two grains separated by a grain boundary. The scalebar corresponds to $40 \mathrm{~nm}$. (h) Overview of the developing contact at this stage (Scalebar: $200 \mathrm{~nm}$ ). (i)-(k) Final breakage. The contact breaks along the remaining grain boundary. The lines numbered 1-4 correspond to the different shapes of the wire. Scalebar $20 \mathrm{~nm}$. Reproduced with permission from Appl. Phys. Lett. 91, 072107 (2007). Copyright 2007 AIP Publishing.

transported in the direction of the current flow and hillocks are formed at the positive electrode (Taychatanapat et al., 2007; Heersche et al., 2007; and Strachan et al., 2008). The overall appearance of the process strongly depends on the initial properties of the wire such as the width. For studies with smaller initial width of the wire, e.g., Strachan et al. (2006), the aspect ratio of the contact is much sharper. If, however, one looks only at the appearance of the notch, the process can be described similarly for wires with small initial width as for wider wires, e.g., Motto et al. (2012) and Stöffler et al. (2012).

As electromigration proceeds, the contact runs over only a single grain separated by grain boundaries. These final stages are influenced by the initial stages of electromigration: In the initial stages of electromigration, grain bending and rotation have been observed that had an impact on the final stages of electromigration (Budiman et al., 2006). In this way, studies of electromigration of single crystals mentioned above contribute to understanding the final breakage.

\section{Recrystallization}

Recrystallization is a controversial issue, because in some studies it is observed (Girod et al., 2012), while in others, it is not observed (Dong and Parviz, 2006; Stöffler et al., 2012; and Heersche et al., 2007), and the origin of these differences has not been studied. If recrystallization is observed, large grains grow at the expense of small grains (Girod et al., 2012) similar to an island ripening process. Here, the change in grain size distribution dominates the electromigration process.

If recrystallization is absent, this indicates that the temperature reached at the center of the junction during the electromigration process was sufficient to allow diffusion processes relevant to electromigration, but too small to allow for recrystallization (Holland et al., 2010). During recrystallization, the atoms of a grain have enough thermal energy to slightly change their position and gain an energetically more favorable position. Since we expect that there is only a small temperature difference between the temperatures needed for the two processes, because nearly similar activation energy barriers are relevant, this observation shows that controlled electromigration allows maintaining the temperature at the contact within a narrow interval. The conditions for recrystallization could thus be traced back to a different temperature distribution potentially caused by differences in the sample lay-out and materials as well as the operating conditions.

\section{Hot-spot and notch formation}

Hot-spot and notch formation has been observed both in SEM studies and in the electrical properties at the same time (Stahlmecke and Dumpich, 2007b). For rather long polycrystalline wires with grain sizes much smaller than the wire width, it has been observed that voids are formed at several positions of the wire. The void area was found to increase linearly with time. Also, the current density increases close to linearly with time during the first $7 \mathrm{~h}$, has a plateau, and then sharply increases before breakdown.

The notch formation can be analyzed in detail by comparing images of the contact before and after electromigration (Stöffler et al., 2012 and Girod et al., 2012), see also Figs. 34 and 35. The process has also been studied in detail by successive SFM imaging during controlled electromigration (Yagi et al., 2015). For wider wires, the notch is mostly formed along grain boundaries as expected. However, in some cases, also grains have disappeared or have been cut. This seems contradictory at first-for a grain to be cut, a relatively sharp temperature profile is needed with a strong variation as a function of lateral position, such that the temperature necessary for diffusion is reached only for part of one grain. On the other hand for grains to disappear fully, 

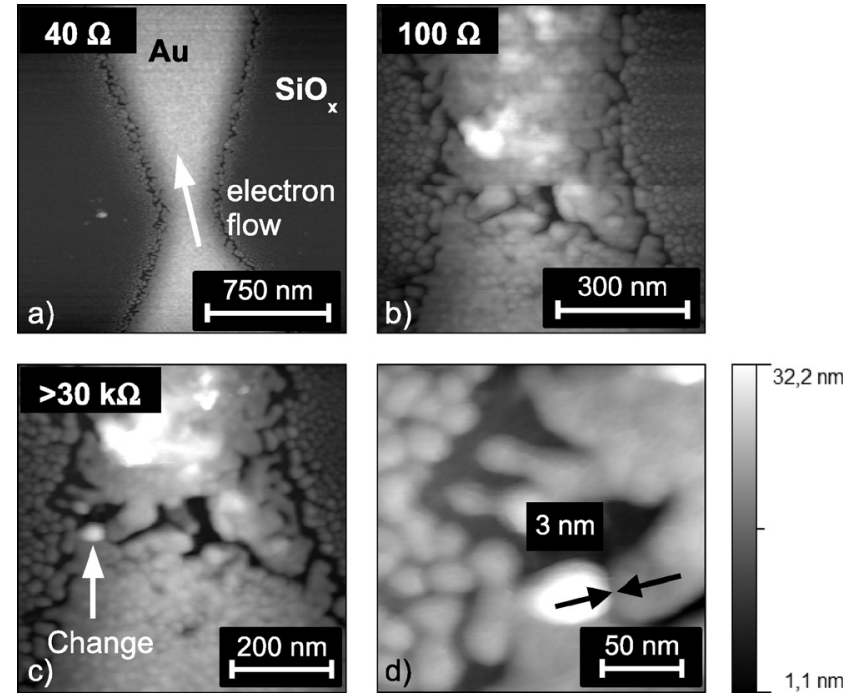

FIG. 34. SFM image of the evolution of a Au thin-film contact under the influence of controlled electromigration. (a) The original structure showed a resistance of $40 \Omega$. (b) After electromigrating the contact, it showed a resistance of $100 \Omega$. The contact has now formed a slit and material has been built up at the cathode side. Further electromigration showed no change at the contact until the contact was deliberately destroyed by applying a large current to it. The result is shown in (c). Image (d) shows a larger view on the region where changes in the contact were observed. Reprinted with permission from Stöffler et al., Phys. Rev. B 85, 033404 (2012). Copyright 2012 American Physical Society.

a rather broad temperature profile on the length scale of the grains is needed (Stöffler et al., 2012).

This apparent contradiction can be resolved by invoking hot-spot formation. First, a relatively large volume is heated and a relatively smooth temperature profile is obtained. The temperature profile varies slowly on the length scale of grains. At a later stage of the thinning process, a relatively small volume is heated with a relatively steep temperature profile. Such a change of heated volume is visible also in the electronic properties of the contact (Fig. 16): in the current versus voltage measurement data of this contact during electromigration, we find that the power needed for electromigration diminishes suddenly in the course of the electromigration process. This is consistent with the information obtained from SFM images: first, the full width of the wire is affected by electromigration as the slit forms and finally only a single grain is affected, see Fig. 34 (Stöffler et al., 2012).
This conclusion can be drawn from Fig. 34, because there is only one spot, the hot-spot, marked by an arrow, where a morphological change is observed in the range between 100 and $30 \mathrm{k} \Omega$.

Transgranular slit formation has been observed previously (Sanchez et al., 1992). The shape evolution, a perturbation, and slit formation have been studied by simulations (Kraft and Arzt, 1995). If a void is sufficiently large-these results are similar to the ones from single-crystalline wires (Mahadevan and Bradley, 1999) — it can change its shape and become a slit, whereas it only moves and preserves its shape if it is small (Kraft and Arzt, 1995). Void formation has also been observed using X-rays where the coherent speckle patterns are analyzed to obtain an image (Takahashi et al., 2009). In another X-ray transmission microscopy study, voids were found to be stable at the position where they were formed near a via (Zschech et al., 2009).

For thinner wires, at the start of the process, the wire is already rather close to a bamboo-like structure where the current runs over a single grain near the weak point of the structure. The orientation of the grains and the microstructure, whether it is random or bamboo-like, was found to be decisive in simulations in $\mathrm{Cu}$ interconnects (Sukharev et al., 2007). Upon downscaling $\mathrm{Cu}$ interconnects, the texture changes from (111) to (110) (Ganesh et al., 2012). Due to the interaction energy of the film with the substrate, for small interconnects a preferential orientation of the grains is observed experimentally (Cao et al., 2013). A correlation between the microstructure and void formation has been established. For this study, electromigration lifetime was tested in realistic situations-i.e., without applying the control scheme described above in Sec. III E 1.

\section{Final breakage}

In all cases where sufficient resolution has been obtained, in the final stage immediately before breakage, the current is shown to run over a single grain (see Fig. 36). Since grain boundaries are no longer available for electromigration, because at the weakest point there is a grain, an unzipping process due to surface electromigration sets in (Strachan et al., 2008). First one atom is removed from a complete row of atoms by thermal excitation. This facilitates the movement of additional atoms in the incomplete layer, (a)

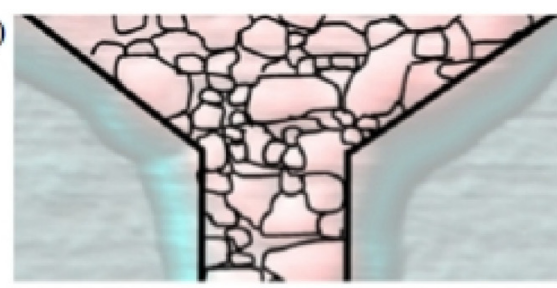

(c)

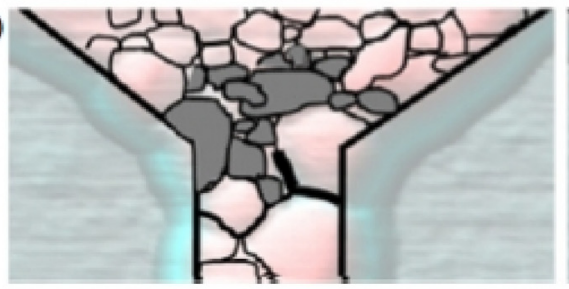

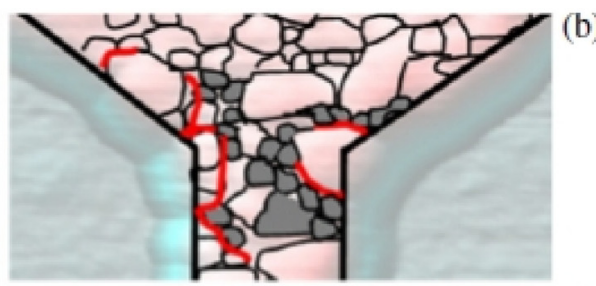

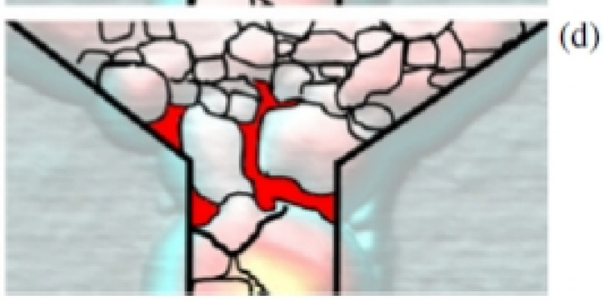

FIG. 35. Development of the structure of a Au nanowire during electromigration as observed with scanning force microscopy. (a) Grain morphology before electromigration. (b) Voids (red) start to form at the cathode side along the grain boundaries near the constriction. (c) Recrystallization causes small grains to disappear and large grains to grow. (d) The grains continue to grow. Reprinted with permission from Girod et al., Nanotechnology 23, 365302 (2012). Copyright 2012 Institute of Physics Publishing. 
a

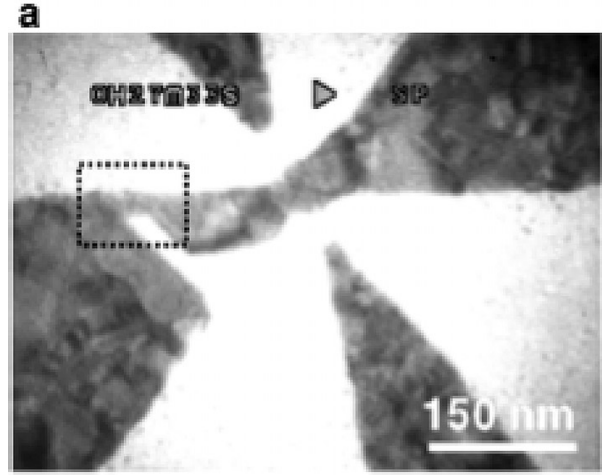

c

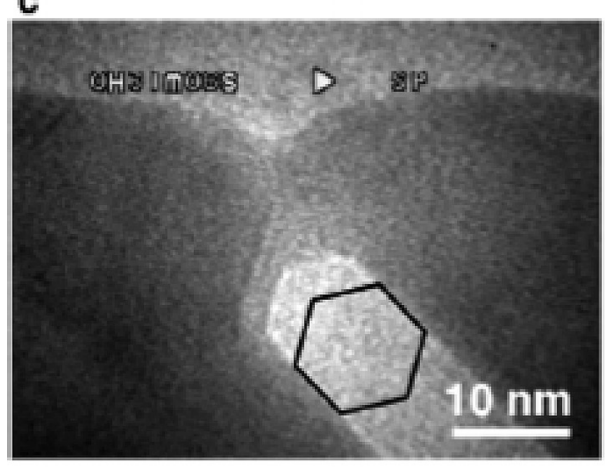

e

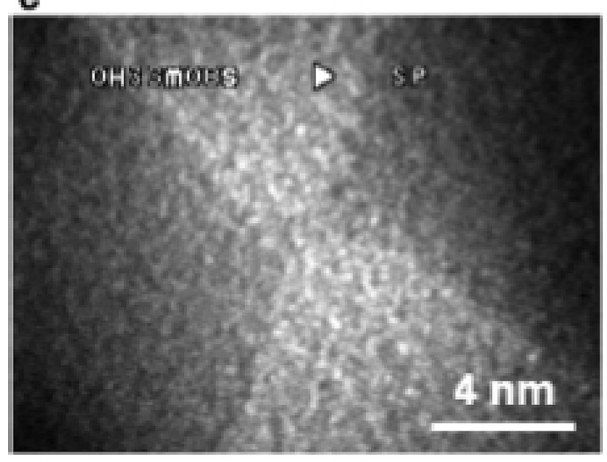

b

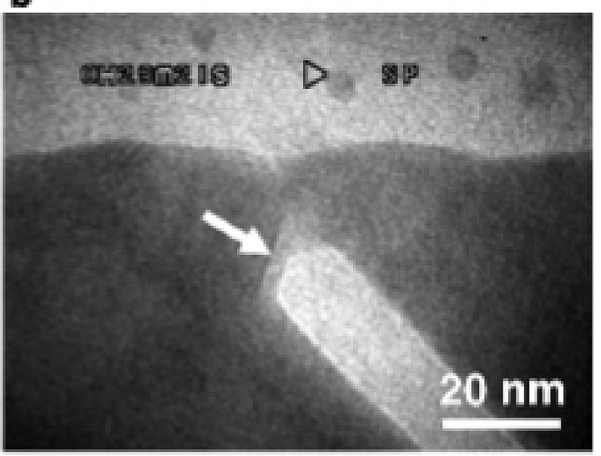

d

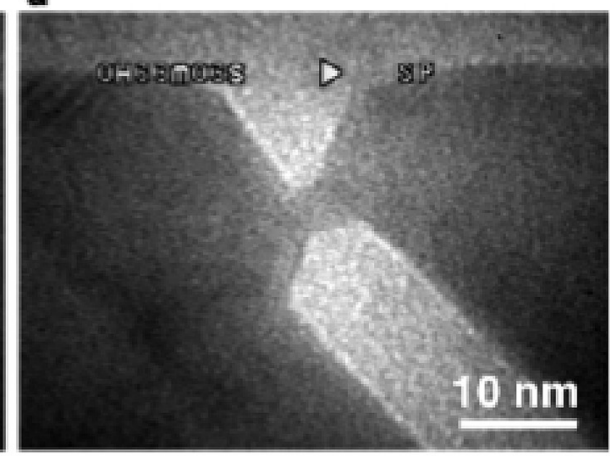

f

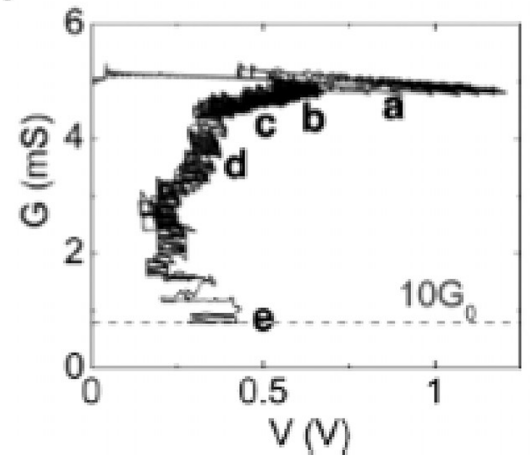

FIG. 36. Final unzipping process as observed in TEM. TEM images (a)-(e) show the evolution of the contact geometry during controlled electromigration as shown in (f). (a) During the first few cycles, a slit is formed. (b) and (c) The point where the Au-metal is still connected clearly shows faceting. The arrow indicates a step edge. (d) Another slit forms at the opposite side. (e) Only few atoms remain in the metallic connection at conductances of $10 G_{0}$. Reprinted with permission from Strachan et al., Phys. Rev. Lett. 100, $056805 \quad$ (2008) Copyright 2008 American Physical Society. i.e., the layer becomes unstable. The atoms of this layer electromigrate until a full row of atoms is removed atom by atom. In another study, a similar process has been observed (Oshima and Kurui, 2013), see also Fig. 37.

The final distance of the electrodes of the broken contact mainly depends on the substrate. On electron-transparent SiN-membranes, the final contact distance is about $5 \mathrm{~nm}$ while on bulk substrates, it is less than $3 \mathrm{~nm}$ (Strachan et al., 2006). The origin of these differences is not clear yet. One possibility is that there are influences of the electron beam on the electromigration process (Stahlmecke and Dumpich, 2007a) if the scanning process is not interrupted for electromigration. Additionally, also heat deposition from the electron beam or carbon contamination could play a role (Oshima et al., 2011). Another possibility is that the differences between bulk and membrane substrates result from the different cooling properties of the substrate.

Compared to standard electromigration testing, it was found that controlled electromigration leads to more symmetric gap geometries compared to simply applying a current for Pt nanowires on $\mathrm{Si}_{3+x} \mathrm{~N}_{4-x}$ membranes (Rudneva et al., 2013). Heersche et al. used a passive breaking scheme, where one simply increases the voltage until the wire breaks and interrupts this process for TEM study. In this case, they did not observe an unzipping process, because in their case, failure occurred at a time-scale shorter than their sampling time (Heersche et al., 2007). They found that the cathode side of the gap was relatively sharp while the anode side had a round shape (Heersche et al., 2007). It was furthermore found that wires thinned to a few conductance quanta continue to narrow after the applied voltage has been removed (O'Neill et al., 2007). No evidence of cluster formation in these junctions was observed. Taychatanapat et al. found that the gap size is smaller when a small series resistance is used (Taychatanapat et al., 2007). Also, the morphology of the leads shows more grains for a larger series resistance.

\section{F. Free-standing thin films}

Free-standing thin films are the type of samples where the highest spatial resolution has been achieved in studies 

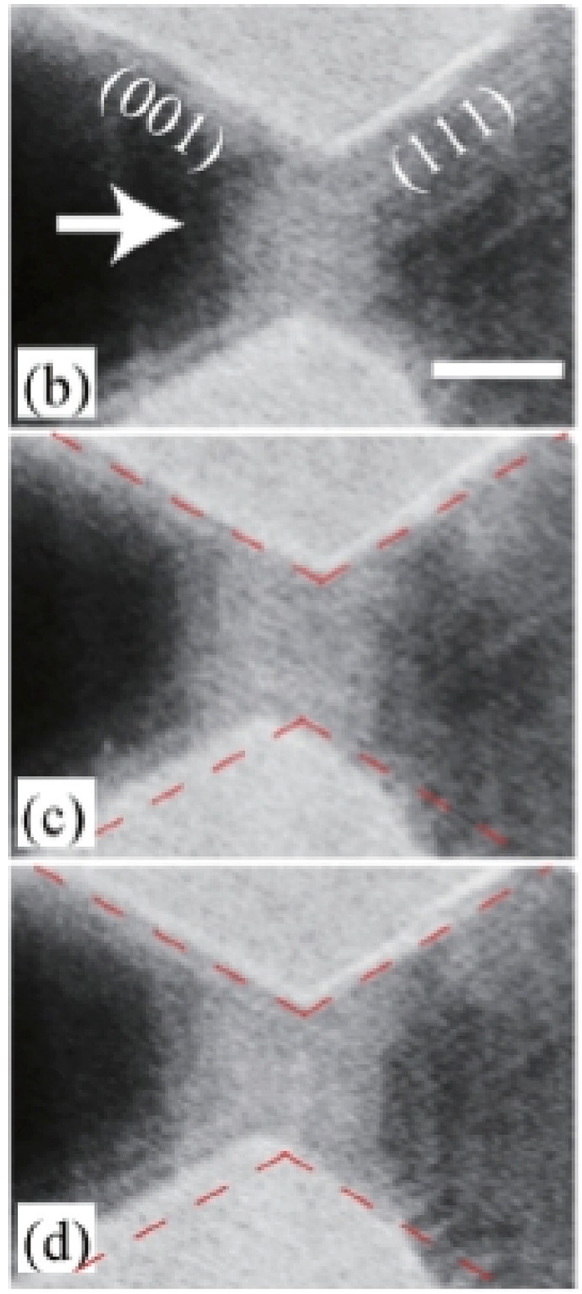

FIG. 37. Evolution of a contact under the influence of an increasing electric current from approx. 0.05 to $0.42 \mathrm{~mA}$. The white arrow indicates the direction of the electron flow. The red dashed line shows in the following images (c) $-(\mathrm{g})$ the shape the contact originally had [in image (b)]. Reprinted with permission from Y. Oshima and Y. Kurui, Phys. Rev. B 87, 081404(R) (2013). Copyright 2013 American Physical Society. employing controlled electromigration. By burning holes into free-standing metallic thin films when a high current density is applied using a TEM, it has been possible to atomically resolve the structure of metallic wires (Kondo and Takayanagi, 2000, 1997; and Rodrigues et al., 2000). In other reports, a STM built inside the TEM allowed to make metallic nanocontacts (Ohnishi et al., 1998). While in some early studies, mono-atomic strands of $\mathrm{Au}$ atoms were observed (Ohnishi et al., 1998), in other studies, thicker wires were formed (Rodrigues et al., 2000 and Kondo and Takayanagi, 2000). The structure of these wires can be unusual and related to magic numbers similar to what is observed in small clusters as has already been discussed in the section on electronic characterization, see Sec. IV B 4. For example, unusual pentagon structures were observed for free-standing $\mathrm{Cu}$ nanowires (Gonzalez et al., 2004a).

One important point of investigation was to clarify the reason of the extraordinary stability of the observed nanowire structures. This stability has been observed experimentally, although it is expected that due to thermal fluctuations, a free-standing nanowire is caused to move in the lateral direction, leading to rupture. In one study, it was claimed that the hexagonal close packed (hcp) surface structure caused an increased stability of $\mathrm{Au}$ wires (Kondo and Takayanagi, 1997). It was even possible to bend a strand of Au atoms.
In another study, nanowires have been bent and stretched, and defects have been introduced by pulling, in particular at low temperatures (Lagos et al., 2011). By pulling, different configurations of $\mathrm{Au}$ wires were obtained from which histograms of the conductance were generated and at the same time their atomic structure was studied by TEM (Kurui et al., 2009). Au nanowires oriented in the [110] direction were observed to give sharp peaks in the conductance histograms, while wires in the [100] and [111] directions gave broad peaks. In view of this extreme stability, it has been discussed whether carbon or oxygen contamination could play a role (Oshima et al., 2011).

The contacts formed in an STM in the focal plane of a TEM were also studied under the influence of an applied electric current (Oshima and Kurui, 2013). For these freestanding contacts, nanogaps of the size of $1-2 \mathrm{~nm}$ were formed due to the remarkable stability of the nanowire formed under these conditions.

\section{LOCAL PHYSICAL QUANTITIES-TEMPERATURE, CURRENT DENSITY, AND SURFACE POTENTIAL}

\section{A. Local temperature distribution}

In the section on controlled electromigration mentioned above (Sec. III E 1), the decisive role of the temperature in electromigration was stressed and estimates of the 
temperature of the junction were given. The abovementioned temperature estimate described by Eq. (21) assumes that electrical and thermal current paths are identical. However, in many cases, heat conduction through the substrate plays a role. A number of efforts have been made to measure (Holm, 1967 and Ward et al., 2008) and calculate the temperature distribution of the device (Durkan, 2007; Kiessig et al., 2014; and Cornelius et al., 2008) in more detail.

Durkan (2007) used a one-dimensional model where it is assumed that heat is created by Joule heating at the same rate everywhere in the wire. This heat is described by $Q=\rho J^{2}$, where $\rho$ is the resistivity of the metal and $J$ the current density. The thermal conductivity of the metal is denoted by $k$. Losses are obtained by heat conduction through the substrate with thermal conductivity $k_{\text {sub }} t$ and $d$ are the thickness of the wire and the substrate (Durkan et al., 1999).

$$
\nabla^{2} T-m^{2} T+\frac{Q}{k}=0 \quad \text { with } \quad m=\sqrt{\frac{k_{\text {sub }}}{k t d}} .
$$

The solution to this equation is given by

$$
T_{\text {wire }}(x)=\frac{Q}{2 m^{2}}\left(1-\frac{1}{2} e^{-m l}\left(e^{m x}+e^{-m x}\right)\right),
$$

where $l$ is the length of the wire. This model has been elaborated and evaluated further for the case where the width of the nanowire is smaller than $1 / m$, the thermal correlation length of the wire, and when the contact plays a significant role (Hunley et al., 2013). A linear description is used for the nanowire, and a radially symmetric spreading is used for the region near the contacts. Another model focuses on determining the cold points where the wire is not heated by the current (Tohmyoh, 2013). The model was used to understand the welding and cutting of ultrathin $\mathrm{Pt}$ and $\mathrm{Ag}$ wires with a diameter in the range of $300 \mathrm{~nm}$ to $1 \mu \mathrm{m}$ and a length between $10 \mu \mathrm{m}$ and several $100 \mu \mathrm{m}$.
A two-dimensional model has been used (Kiessig et al., 2014), Fig. 38. By a careful comparison of measurements and simulations, it can be shown that a good thermal coupling to the substrate plays an important role in the temperature balance (Durkan et al., 1999; Kiessig et al., 2014; and Yang et al., 2005).

For the ballistic regime, scattering of the electrons is reduced in the contact region. The electrons are transmitted across the contact in a non-equilibrium hot state. Within an area described by the mean free path taken in both directions of the contact, they scatter and deposit their energy (Datta, 1995). The ballistic nanocontact will be heated by heat diffusion.

For monoatomic Au wires, the problem has been treated theoretically (Todorov, 1998; Todorov et al., 2001; Tsutsui et al., 2006; and Smit et al., 2004). The temperature of the junction at breakage is $T=\left(T_{0}^{4}+T_{U}^{4}\right)^{1 / 4}$ with $T_{U}=\gamma$ $\sqrt{l\left|U_{C}\right|}$, where $\gamma$ is estimated to be about 60 or $75 \mathrm{~K} /\left(\mathrm{V}^{1 / 2}\right.$ $\mathrm{nm}^{1 / 2}$ ) based on values obtained for typical metals and $l$ is the length of the nanowire.

While calculations offer detailed theoretical information about the local temperature of the junction, it is a technical challenge to measure the local temperature of the junction. One approach uses the blackbody radiation (Ward et al., 2008). Temperatures up to $1000 \mathrm{~K}$ are measured on a $\mathrm{Pt}$ bow-tie junction. These temperatures are high if one compares with other estimates and calculations that are usually done for $\mathrm{Au}$, but in agreement with what is expected for this material, see also Table II. The local temperature distribution has been characterized using scanning thermal microscopy (Kim et al., 2012). In this approach in a standard SFM set-up, a tip with a layered structure that serves as a thermoelement is used. The voltage measured between the two electrodes of this microfabricated thermoelement gives-after calibration - the local temperature of the region imaged with the SFM. During electromigration of Au nanowires, a temperature up to $400^{\circ}$ is observed at the endpoint of the cycles (Jeong et al., 2014). This temperature is centered around a
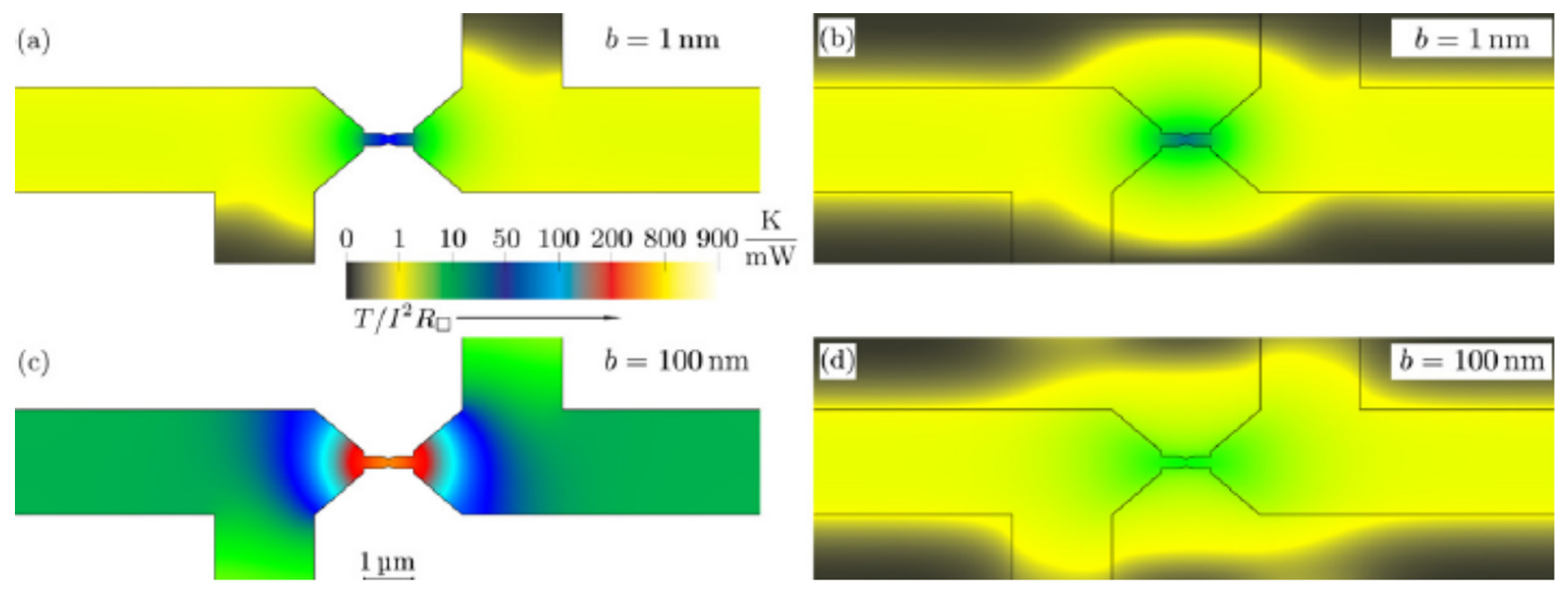

FIG. 38. Maps of the normalized temperature for the metallic structure (a) and (c) and for the substrate (Si) oxide-barrier interface (b) and (d). $b$ refers to the thickness of the oxide-barrier. Reprinted with permission from Kiessig et al., New J. Phys. 16, 013017 (2014). Copyright 2014 Institute of Physics Publishing. 
hot-spot and decreases in the course of electromigration. Not only the lattice temperature but also the effective electronic temperature in a Au nanojunction as an out-of-equilibrium system has been measured using surface-enhanced Raman emission (Ward et al., 2011). The incident light creates electron-hole excitations in the metal, leading to Stokes scattering, whereas anti-Stokes scattering requires excited electrons above the Fermi level. It has been found that the effective temperature can reach values up to $2000 \mathrm{~K}$ for vibrational modes out of equilibrium. Pumping of vibrational modes as well as shifts due to the applied bias are observed using Raman experiments under a current flow (Natelson et al., 2013). Another group has investigated the local temperature of the junction as a function of the temperature of the environment and found a parabolic dependence of the maximum local temperature on the temperature of the environment (Xiang et al., 2014).

Whether heat generation is unequal for both electrodes and in addition differs for positive and negative currents in a $\mathrm{Au}-\mathrm{Au}$ nanojunction has been studied theoretically and experimentally (Lee et al., 2013). It has been found that there is no asymmetry for positive and negative biases, as expected and in contrast to a molecular junction. In line with this observation, the computed transmission function shows a weak energy dependence around the Fermi energy. The thermopower vanishes (Pauly et al., 2011). In another study, it has been proposed that even a symmetric biased nanocontact should have an asymmetric temperature distribution in contrast to the linear Landauer-Büttiker theory (Lü et al., 2015). Also, an experimental study has found a temperature difference for Au junctions (Tsutsui et al., 2012).

A set of several parallel nanoelectrodes each made from two different materials were used for electromigration to make large area junctions for thermoelectric devices (Tanielian et al., 2011). The electrodes were placed on two glass substrates and bonded together. It was shown that also for such devices, tunneling gaps can be fabricated by electromigration.

\section{B. Local current density and potential near nanocontacts}

The local current density is a quantity that is difficult to access experimentally but is important for the structural evolution of nanocontacts during electromigration. Due to current crowding near defects, the local wind force can vary and influence the local effects of electromigration. The local current density and potential have been predicted for arbitrary atomistic configurations using a tight-binding model (Todorov, 2002).

Efforts have been taken to determine experimentally the local current density using magnetic force microscopy, by detecting the magnetic fields generated by the sample (Rous et al., 2004). Although so far the resolution is not expected to reach atomic dimensions, this is a promising approach for metals, because other methods of experimentally detecting current paths are lacking.

In semiconductors, where the Fermi wavelength is much larger than the size of an atom, and conductance quantization has been clearly observed (van Wees et al., 1988 and
Wharam et al., 1988), a signature of the local current density has been determined using scanning force microscopy, by influencing the local Fermi level by applying a voltage with the tip (Topinka et al., 2000). While the tip was placed above a semiconducting nanocontact sample with variable size of the nanocontact, the tip was scanned parallel to the sample plane and a voltage was applied to the tip. The local Fermi level of the sample region under tip was influenced and consequently also electronic transport measured through the sample during scanning was influenced. It was found that the area where the current can be influenced by the presence of a SFM tip shows one lobe for one conductance quantum, two lobes for two conductance quanta, and three lobes for three conductance quanta (Topinka et al., 2000).

The local surface potential in Au nanocontacts has been imaged using Kelvin probe force microscopy, a technique that allows to determine the local electrochemical potential (Arnold et al., 2017). In this way, it has been shown that electrically disconnected grains occur in the sample after electromigration. These could give rise to Coulomb blockade effects.

\section{NANOCONTACTS OF ALLOYS}

Alloys are discussed separately, because their structural and electromigration properties strongly differ from the ones of pure metals. Electromigration of alloys has been studied for a long time with the aim of making interconnects more electromigration-resistant. Concerning the electronic transport properties in the ballistic regime, alloys of transition metals and noble metals have been investigated (Heemskerk et al., 2003). At low bias voltages, the histograms resemble those of the noble metals, while they resemble those of the transition metals at high bias voltages. The authors attribute this behavior to a current-induced transition of the nanoscalemetallic contact where the role of the different types of atoms of the alloy changes.

\section{A. Electromigration of alloys}

Dissolving a solute in a metal solvent usually enhances electromigration. However, for some important special cases, it can also retard electromigration and therefore it is of significant technological importance (Hau-Riege, 2004). For an overview, see Ho and Kwok (1989).

Enhancing electromigration by adding a solute is the result of two competing effects: The presence of a solute changes the effective charge number $Z^{*}$, because the atom (vacancy) flow for different atom types responds differently to the driving forces. This usually leads to retarding electromigration as found for many solutes for $\mathrm{Ag}, \mathrm{Au}, \mathrm{Al}$, and $\mathrm{Cu}$ solvents from an analysis of jump frequency data using a model (Ho and Kwok, 1989). In addition, the solute changes the diffusivity, usually leading to an enhancement of electromigration. In general, the second effect outweighs the first, see also the table in Ho and Kwok (1989) that covers a considerable range of solutes and solvents. On the other hand, $\mathrm{Cu}$ addition in $\mathrm{Al}$ is known to retard electromigration, whereas $\mathrm{Al}$ addition in $\mathrm{Cu}$ is known to enhance electromigration. The details of this process have led to the conclusion 
that the effect of alloying depends on whether the solute is added to the grains of the solvent or rather to the grain boundaries (Ho and Kwok, 1989).

A model has been developed to explain under which circumstances a solute can retard electromigration (Dekker et al., 2001). It was found that the flux of solvent atoms can be reversed compared to the flux of solute atoms under particular circumstances. For this to occur, the solute concentration must be sufficiently large, because the vacancy must make many jumps in the vicinity of the solute atom for the influence of the solute on the electromigration to be of significance. Here, the motion of vacancies is considered instead of the motion of solvent atoms to simplify the picture. In addition, the relation between electromigration driving forces of solute and host must be sufficiently large such that it is energetically favorable for a solute and a vacancy to move in the direction of the driving force, while two solvent atoms move in the opposite direction. This is obtained by choosing a solute with appropriate $Z^{*}$. It has been discussed whether $\mathrm{Sn}$ as a solute in $\mathrm{Cu}$ lines could retard electromigration (Hau-Riege, 2004).

$\mathrm{Mn}$ and $\mathrm{Al}$ solutes in $\mathrm{Cu}$ have been investigated $(\mathrm{Hu}$ et al., 2012 and Oates, 2015). It was found that both elements generally enhance electromigration. Only for very low concentrations of $\mathrm{Mn}$, a retardation has been observed. Bamboolike grains played a crucial role in this retardation, because a blocking at grains was observed. It was concluded that the grains were below the Blech length in size and thus counterbalance the stress build up in the lines. Also, the influence of $\mathrm{Al}$ doping in $\mathrm{Cu}$ at low concentrations has been investigated (Yokogawa and Tsuchiya, 2007). A model is tested which discusses depletion of the dopant at the cathode. It is found that $\mathrm{Al}$ affects the electromigration-induced diffusion of $\mathrm{Cu}$ but not the electromigration driving force. In another study, the effect of $\mathrm{Al}, \mathrm{Co}$, and $\mathrm{Mn}$ doping on $\mathrm{Cu}$ lines has been investigated (Arnaud et al., 2013). It was found that the electromigration process was not slowed down, but the incubation time needed for it to start was increased, leading to an overall slower process. In this study, the incubation time was defined as the time after which a sharp increase of the resistance was first observed followed by a linear increase with time.

Electromigration can also lead to interdiffusion at interfaces or phase separation in alloys. When the current was flowing from $\mathrm{Ni}$ to $\mathrm{Cu}$, a marked increase of the interdiffusion was observed as compared to the case where the current was flowing from $\mathrm{Cu}$ to $\mathrm{Ni}$ (Zhao et al., 2007).

\section{B. Structural evolution during electromigration of alloys}

Concerning the structural evolution, for alloys, a clearly distinct behavior compared to pure elements is observed using TEM (Kozlova et al., 2013) as well as scanning force microscopy (Rudneva et al., 2013). Instead of a notch, voids are formed. These voids grow and coalesce such that larger holes are formed until the contact finally breaks. Also here, the process starts at grain boundaries. This behavior can be explained by a local variation of the binding energy of the atoms with the grain boundaries due to the microstructure of the alloy. At the beginning of electromigration thinning, the junction is heated over a relatively large area comprising local minima of the binding energy. At the locations of these minima, electromigration first starts. In the particular example shown in Fig. 39, the electromigration started near the end of the bridge possibly due to the atomic flux divergences occurring near this position. Similarly as for many pure metals, for Pd-Pt (10 at. \%) alloys, the mass transport occurs in the direction of the flow of electrons. Significant recrystallization is observed.

The behavior can be reversed by inverting the direction of the current flow (Kozlova et al., 2013). In addition, the influence of an alternating current (AC) on the junction has been studied. For an AC current, material transfer starts not at the cathode but at the center of the bridge. This indicates that for an AC current Joule heating, the associated mechanical stress caused by thermal expansion has a stronger influence on electromigration compared to singularities in the flow of atoms as observed for a DC current. In the center of the bridge, it is expected that the wire is hotter than near the contacts (Kozlova et al., 2013).

From the structural properties of the electromigration process observed here and the large holes that appear, it is clear that tunneling is not expected at the initial and intermediate electromigration stages, because the holes are much too large to allow tunneling. This is in agreement with observations for permalloy that tunneling is not observed and also
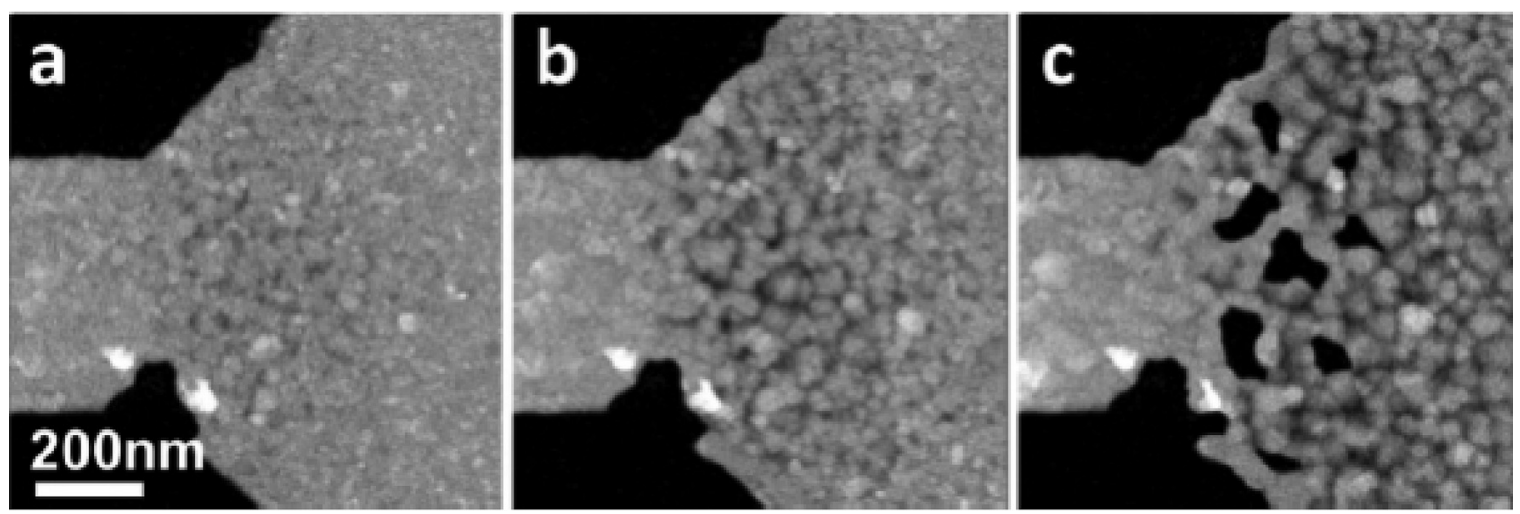

FIG. 39. Evolution of a thin film of a Pd-Pt (10 at. \%) alloy near the cathode side during electromigration. Instead of forming a notch, holes are formed. Reprinted with permission from Kozlova et al., Nanotechnology 24, 505708 (2013). Copyright 2013 Institute of Physics Publishing. 
the $\mathrm{d} R / \mathrm{d} U$ characteristics do not show non-linearities even at rather high resistance values (von Bieren et al., 2013).

In alloys, precipitation at the grain boundaries may play a significant role, as has been found for $\mathrm{Sn}$ in a Ni/Sn/Ni test structure (Huang et al., 2013). Also, $\mathrm{Cu}$ and Ag solutes with a Sn solvent have been found to segregate and influence grain boundary diffusivity (Sellers et al., 2011).

\section{CONCLUSIONS}

Through electromigration methods on the one hand and TEM fabrication methods on the other hand, a rich variety of new studies of the structure of nanocontacts have become possible. Knowing the structure is fundamental for understanding the electronic transport properties of nanocontacts. In practice, studying nanocontacts electronically is much easier than studying their structure. Controlled electromigration allows to keep the temperature of the contact during electromigration within a narrow range and to take control over the self-accelerating nature of hot-spot and nanocontact formation. In addition, the development of a detailed theoretical understanding of the electromigration forces has greatly advanced this field. Electronic studies of nanocontacts generate assumptions of the structure that cannot easily be checked independently. These assumptions are in many cases the only information known on the contact's structure. With highresolution SFM and TEM methods, the details of the nanocontact formation that depend on the local micro- and nanostructure of the metal are now accessible. For singlecrystalline nanowires, the usually dominating grain boundary electromigration is absent making such nanowires stable to electromigration. The formation of hot-spots by growing slits and the structural evolution of grains have been studied.

\section{ACKNOWLEDGMENTS}

I thank G. Goll, J. Hauser, M. Marz, C. Pérez León, R. Schäfer, E. Scheer, Th. Schimmel, and H. v. Löhneysen for critically reading the manuscript and for useful discussions and support. Financial support from the European Research Council through the Starting Grant NANOCONTACTS (No. 239838) and from the ministry of science, research and arts, Baden-Württemberg, in the framework of the BrigitteSchlieben-Lange program is gratefully acknowledged.

Agrait, N., Levy Yeyati, A., and van Ruitenbeek, J. M., Phys. Rep. 377, 81 (2003).

Albrecht, T. R., Grütter, P., Horne, D., and Rugar, D., J. Appl. Phys. 69, 668 (1991).

Anaya, A., Korotkov, A. L., Bowman, M., Waddell, J., and Davidovic, D., J. Appl. Phys. 93, 3501 (2003).

Anselmetti, D., Richmond, T., Baratoff, A., Borer, G., Dreier, M., Bernasconi, M., and Güntherodt, H.-J., Europhys. Lett. 25, 297 (1994).

Aono, M. and Hasegawa, T., Proc. IEEE 98, 2228 (2010).

Aradhya, S. V. and Venkataraman, L., Nat. Nanotechnol. 8, 399 (2013).

Argento, C. and French, R. H., J. Appl. Phys. 88, 6081 (1996).

Armstrong, J. N., Schaub, R. M., Hua, S. Z., and Chopra, H. D., Phys. Rev. B 82, 195416 (2010).

Arnaud, L., Lamontagne, P., Bana, F., Friec, Y. L., and Waltz, P., Microelectron. Eng. 107, 145 (2013).

Arnold, D., Marz, M., Schneider, S., and Hoffmann-Vogel, R., Nanotechnology 28, 055206 (2017).

Arzubiaga, L., Golmar, F., Llopis, R., Casanova, F., and Hueso, L. E., Appl.

Phys. Lett. 102, 193103 (2013).
Bakker, D. J., Noat, Y., Yanson, A. I., and van Ruitenbeek, J. M., Phys. Rev. B 65, 235416 (2002).

Barth, J. V., Surf. Sci. Rep. 40, 75 (2000).

Barzilai, S., Tavazza, F., and Levine, L. E., Modell. Simul. Mater. Sci. Eng. 21, 025004 (2013).

Beenakker, C. W. J., Phys. Rev. B 44, 1646 (1991).

Besold, J., Kunze, R., and Matz, N., J. Vac. Sci. Technol. B 12, 1764 (1994). Bevan, K. H., Guo, H., Williams, E. D., and Zhang, Z., Phys. Rev. B 81, 235416 (2010).

Bevan, K. H., Zhu, W., Stocks, G. M., Guo, H., and Zhang, Z., Phys. Rev. B 85, 235421 (2012).

Beyne, S., Croes, K., Wolf, I. D., and Tökei, Z., J. Appl. Phys. 119, 184302 (2016).

von Bieren, A. C., Patra, A. K., Krzyk, S., Rhensius, J., Heyderman, L. J., Hoffmann-Vogel, R., and Kläui, M., Phys. Rev. Lett. 110, 067203 (2013).

Bietsch, A. and Michel, B., Appl. Phys. Lett. 80, 3346 (2002).

Binnig, G. and Rohrer, H., Helv. Phys. Acta 55, 726 (1982).

Binnig, G., Rohrer, H., Gerber, Ch., and Weibel, E., Phys. Rev. Lett. 49, 57 (1982).

Black, J. R., in Proceedings 6th Annual International Reliablity Physics Symposium (1967), p. 148.

Blech, I. A., J. Appl. Phys. 47, 1203 (1976).

Blech, I. A. and Herring, C., Appl. Phys. Lett. 29, 131 (1976).

Blech, I. A. and Meieran, E. S., Appl. Phys. Lett. 11, 263 (1967).

Blech, I. A. and Meieran, E. S., J. Appl. Phys. 40, 485 (1969).

Blech, V., Nobuyuki, T., and Kim, B., J. Vac. Sci. Technol. B 24, 55 (2006).

Bly, D. N. and Rous, P. J., Phys. Rev. B 53, 13909 (1996).

Bode, N., Kusminskiy, S. V., Egger, R., and von Oppen, F., Phys. Rev. Lett. 107, 036804 (2011).

Bolotin, K. I., Kuemmeth, F., Pasupathy, A. N., and Ralph, D. C., Appl. Phys. Lett. 84, 3154 (2004).

Bondarchuk, O., Cullen, W. G., Degawa, M., and Williams, E. D., Phys. Rev. Lett. 99, 206801 (2007).

Bosvieux, C. and Friedel, J., J. Phys. Chem. Solids 23, 123 (1962).

Bowler, D. R., J. Phys.: Condens. Matter 16, R721 (2004).

Bowman, M., Anaya, A., Korotkov, A. L., and Davidovic, D., Phys. Rev. B 69, 205405 (2004).

Brandbyge, M., Stokbro, K., Taylor, J., Mozos, J.-L., and Ordejón, P., Phys. Rev. B 67, 193104 (2003).

Braun, K.-F., Soe, W.-H., Flipse, C. F. J., and Rieder, K.-H., Appl. Phys. Lett. 90, 023118 (2007).

Briner, B. G., Doering, M., Rust, H.-P., and Bradshaw, A. M., Science 278, 257 (1997).

Brinkman, W. F., Dynes, R. C., and Rowell, J. M., J. Appl. Phys. 41, 1915 (1970).

Brouër, S., Weiss, G., and Weber, H. B., Europhys. Lett. 54, 654 (2001).

Brune, H., Surf. Sci. Rep. 31, 121 (1998).

Budiman, A. S., Nix, W. D., Tamura, N., Valek, B. C., Gadre, K., Maiz, J., Spolenak, R., and Patel, J. R., Appl. Phys. Lett. 88, 233515 (2006).

Campbell, J. M. and Knobel, R. G., Appl. Phys. Lett. 102, 023105 (2013).

Cao, L., Ganesh, K. J., Zhang, L., Aubel, O., Hennesthal, C., Hauschildt, M., Ferreira, P. J., and Ho, P. S., Appl. Phys. Lett. 102, 131907 (2013).

Castro, D. T., Hoofman, R. J. O. M., Michelon, J., Gravesteijn, D. J., and Bruynseraede, C., J. Appl. Phys. 102, 123515 (2007).

Ceric, H. and Selberherr, S., Mater. Sci. Eng. R 71, 53 (2011).

Chen, K., Tamura, N., Valek, B. C., and Tu, K. N., J. Appl. Phys. 104, 013513 (2008a).

Chen, K.-C., Liao, C.-N., Wu, W.-W., and Chen, L.-J., Appl. Phys. Lett. 90, 203101 (2007).

Chen, K.-C., Wu, W.-W., Liao, C.-N., Chen, L.-J., and Tu, K. N., Science 321, 1066 (2008b).

Cheng, Y.-L., Chang, W.-Y., and Wang, Y.-L., J. Vac. Sci. Technol. B 28, 1322 (2010).

Cheng, Y.-L., Leon, K.-W., Huang, J.-F., Chang, W.-Y., Chang, Y.-M., and Leu, J., Microelectron. Eng. 114, 12 (2014).

Cho, J. and Thompson, C. V., Appl. Phys. Lett. 54, 2577 (1989).

Cho, Z.-S., Lee, J., Lim, M. K., Gan, C. L., and Thompson, C. V., J. Appl. Phys. 110, 033505 (2011).

Choi, Z.-S., Mönig, R., and Thompson, C. V., J. Appl. Phys. 102, 083509 (2007).

Cornelius, T. W., Picht, O., Müller, S., Neumann, R., Völklein, F., Karim, S., and Duan, J. L., J. Appl. Phys. 103, 103713 (2008). 
Coura, P. Z., Legoas, S. B., Moreira, A. S., Sato, F., Rodrigues, V., Dantas, S. O., Ugarte, D., and Galvao, D. S., Nano Lett. 4, 1187 (2004).

Cross, G., Schirmeisen, A., Grütter, P., and Dürig, U. T., Nat. Mater. 5, 370 (2006).

Cross, G., Schirmeisen, A., Stalder, A., Grütter, P., Tschudy, M., and Dürig, U., Phys. Rev. Lett. 80, 4685 (1998).

Csonka, S., Halbritter, A., Mihály, G., Jurdik, E., Shklyarevskii, O. I., Speller, S., and van Kempen, H., Phys. Rev. Lett. 90, 116803 (2003).

Cuevas, J. C. and Scheer, E., Molecular Electronics (World Scientific, New Jersey, 2010).

Cunningham, B., Todorov, T. N., and Dundas, D., Phys. Rev. B 90, 115430 (2014).

Damle, P., Rakshit, T., Paulsson, M., and Datta, S. S., IEEE Trans. Nano 1 145 (2002).

Das, A. K. and Peierls, R. E., J. Phys. C 6, 2811 (1973).

Datta, S., Electronic Transport in Mesoscopic Systems (Cambridge University Press, Cambridge, 1995).

Dekker, J. P., Gumbsch, P., Arzt, E., and Lodder, A., Phys. Rev. B 59, 7451 (1999).

Dekker, J. P., Lodder, A., and van Ek, J., Phys. Rev. B 19, 12167 (1997).

Dekker, J. P., Volkert, C. A., Arzt, E., and Gumbsch, P., Phys. Rev. Lett. 87, 035901 (2001).

Doi, K., Iguchi, K., Nakamura, K., and Tachibana, A., Phys. Rev. B 67, 115124 (2003).

Dong, J. and Parviz, B. A., Nanotechnology 17, 5124 (2006).

Dreher, M., Pauly, F., Heurich, J., Cuevas, J. C., Scheer, E., and Nielaba, P., Phys. Rev. B 72, 075435 (2005).

Dundas, D., McEniry, E. J., and Todorov, T. N., Nat. Nanotechnol. 4, 99 (2009).

Durkan, C., Current at the Nanoscale (Imperial College Press, London, 2007).

Durkan, C., Schneider, M. A., and Welland, M. E., J. Appl. Phys. 86, 1280 (1999).

Durkan, C. and Welland, M. E., Crit. Rev. Solid State Mater. Sci. 25, 1 (2000a)

Durkan, C. and Welland, M. E., Phys. Rev. B 61, 14215 (2000b).

Dwyer, V. M., J. Appl. Phys. 107, 103718 (2010).

Egger, S., Ilie, A., Fu, Y., Chongsathien, J., Kang, D.-J., and Welland, M. E., Nano Lett. 5, 15 (2005).

Eigler, D. M. and Schweizer, E. K., Nature 344, 524 (1990).

Esen, G. and Fuhrer, M. S., Appl. Phys. Lett. 87, 263101 (2005).

Fernández-Alcázar, L. J., Bustos-Marún, R. A., and Pastawski, H. M., Phys. Rev. B 92, 075406 (2015).

Fiks, V. B., Fiz. Tverd. Tela (Leningrad) 1, 16 (1959a).

Fiks, V. B., Sov. Phys. Solid State 1, 14 (1959b).

Filippi, R. G., Wachnik, R. A., Eng, C.-P., Chidambarrao, D., Wang, P.-C., White, J. F., Korhonen, M. A., Shaw, T. M., Rosenberg, R., and Sullivan, T. D., J. Appl. Phys. 91, 5787 (2002).

Fischbein, M. D. and Drndic, M., Appl. Phys. Lett. 88, 063116 (2006).

Fishlock, T. W., Oral, A., Egdell, R. G., and Pethica, J. B., Nature 404, 743 (2000).

Fostner, S., Burke, S. A., Topple, J., Mativetsky, J. M., Beerens, J., and Grutter, P., Microelectron. Eng. 87, 652 (2010).

Frigo, S. P., Levine, Z. H., and Zaluzec, N. J., Appl. Phys. Lett. 81, 2112 (2002).

Fuechsle, M., Miwa, J. A., Mahapatra, S., Ryu, H., Lee, S., Warschkow, O., Hollenberg, L. C. L., Klimeck, G., and Simmons, M. Y., Nat. Nanotechnol. 7, 242 (2012).

Fulton, T. A. and Dolan, G. J., Phys. Rev. Lett. 59, 109 (1987).

Ganesh, K. J., Darbal, A. D., Rajasekhara, S., Rohrer, G. S., Barmak, K., and Ferreir, P. J., Nanotechnology 23, 135702 (2012).

Gao, B., Rudneva, M., McGarrity, K. S., Xu, Q., Prins, F., Thijssen, J. M., Zandbergen, H., and van der Zant, H. S. J., Nanotechnology 22, 205705 (2011).

Garcia, R. and Perez, R., Surf. Sci. Rep. 47, 197 (2002).

Gardinowski, G., Schmeidel, J., Pfnür, H., Block, T., and Tegenkamp, C., Appl. Phys. Lett. 89, 063120 (2006).

Gärtner, C., Hoffmann, R., Perez-Willard, F., Sauter, M., Sürgers, C., and Löhneysen, H. V., Rev. Sci. Instrum. 77, 026101 (2006).

Giessibl, F. J., Science 267, 68 (1995).

Giessibl, F. J., Rev. Mod. Phys. 75, 949 (2003).

Gimzewski, J. K. and Möller, R., Phys. Rev. B 36, 1284 (1987).

Girod, S., Bubendorff, J.-L., Montaigne, F., Simon, L., Lacour, D., and Hehn, M., Nanotechnology 23, 365302 (2012).
Gonzalez, J. C., Rodrigues, V., Bettini, J., Rego, L. G. C., Rocha, A. R., Coura, P. Z., Dantas, S. O., Sato, F., Galvao, D. S., and Ugarte, D., Phys. Rev. Lett. 93, 126103 (2004a)

Gonzalez, J. I., Lee, T.-H., Barnes, M. D., Antoku, Y., and Dickson, R. M., Phys. Rev. Lett. 93, 147402 (2004b).

Gorter, C. J., Physica 17, 777 (1951).

Gramich, J., Brenner, P., Sürgers, C., Löhneysen, H. v., and Goll, G., Phys. Rev. B 86, 155402 (2012).

Guggisberg, M., Bammerlin, M., Loppacher, C., Pfeiffer, O., Abdurixit, A., Barwich, V., Bennewitz, R., Baratoff, A., Meyer, E., and Güntherodt, H. J., Phys. Rev. B 61, 11151 (2000).

Gundlach, K. H., Solid-State Electron. 9, 949 (1966).

Guo, H., Martrou, D., Zambelli, T., Polesel-Maris, J., Piednoir, A., Dujardin, E., Gauthier, S., van den Boogaart, M. A. F., Doeswijk, L. M., and Brugger, J., Appl. Phys. Lett. 90, 093113 (2007).

Hadeed, F. O. and Durkan, C., Appl. Phys. Lett. 91, 123120 (2007).

Haekkinen, H., Barnett, R. N., Scherbakov, A. G., and Landman, U., J. Phys. Chem. B 104, 9063 (2000).

Halbritter, A., Csonka, S., Kolesnychenko, O. Y., Mihaly, G., Shklyarevskii, O. I., and van Kempen, H., Phys. Rev. B 65, 045413 (2002).

Han, J. H., Song, K., Radhakrishnan, S., Oh, S. H., and Lee, C. H., Appl. Phys. Lett. 101, 183106 (2012).

Hansen, K., Laegsgaard, E., Stensgaard, I., and Besenbacher, F., Phys. Rev. B 56, 2208 (1997).

Hansen, K., Nielsen, S. K., Brandbyge, M., Laegsgaard, E., Stensgaard, I., and Besenbacher, F., Appl. Phys. Lett. 77, 708 (2000).

Hasmy, A., Medina, E., and Serena, P. A., Phys. Rev. Lett. 86, 5574 (2001).

Hau-Riege, C. S., Microelectron. Reliab. 44, 195 (2004).

Hauser, J., Schwichtenberg, J., Marz, M., Sürgers, C., Seiler, A., Gerhards, U., Messerschmidt, F., Hensel, A., Dittmeyer, R., Löhneysen, H. V., and Hoffmann-Vogel, R., Appl. Phys. A 122, 1068 (2016).

Heemskerk, J. W. T., Noat, Y., Bakker, D. J., van Ruitenbeek, J. M., Thijsse, B. J., and Klaver, P., Phys. Rev. B 67, 115416 (2003).

Heersche, H. B., Lientschnig, G., O’Neill, K., van der Zant, H. S. J., and Zandbergen, H. W., Appl. Phys. Lett. 91, 072107 (2007).

Herz, M. and Scheer, E., Appl. Phys. Lett. 108, 023103 (2016).

Ho, P. S. and Kwok, T., Rep. Prog. Phys. 52, 301 (1989).

Hoekstra, J., Sutton, A. P., Todorov, T. N., and Horsfield, A. P., Phys. Rev. B 62, 8568 (2000).

Hoffmann, R., in Noncontact Atomic Force Microscopy, edited by R. W. S. Morita and E. Meyer (Springer, Berlin Heidelberg, 2009), Vol. 2.

Hoffmann, R., Kantorovich, L. N., Baratoff, A., Hug, H. J., and Güntherodt, H.-J., Phys. Rev. Lett. 92, 146103 (2004).

Hoffmann, R., Lantz, M. A., Hug, H. J., van Schendel, P. J. A., Kappenberger, P., Martin, S., Baratoff, A., and Güntherodt, H.-J., Phys. Rev. B 67, 085402 (2003).

Hoffmann, R., Weissenberger, D., Hawecker, J., and Stöffler, D., Appl. Phys. Lett. 93, 043118 (2008).

Holland, T. B., Thron, A. M., Bonifacio, C. S., Mukherjee, A. K., and van Benthem, K., Appl. Phys. Lett. 96, 243106 (2010).

Holm, R., Electric Contacts (Springer-Verlag, Berlin, 1967).

Homoth, J., Wenderoth, M., Druga, T., Winking, L., Ulbrich, R. G., Bobisch, C. A., Weyers, B., Bannani, A., Zubkov, E., Bernhart, A. M., Kaspers, M. R., and Möller, R., Nano Lett. 9, 1588 (2009).

Houck, A. A., Labaziewicz, J., Chan, E. K., Folk, J. A., and Chuang, I. L., Nano Lett. 5, 1685 (2005).

Hu, C.-K., Gignac, L., Liniger, E., Herbst, B., Rath, D. L., Chen, S. T., Kaldor, S., Simon, A., and Tseng, W.-T., Appl. Phys. Lett. 83, 869 (2003).

Hu, C.-K., Ohm, J., Gignac, L. M., Breslin, C. M., Mittal, S., Bonilla, G., Edelstein, D., Rosenberg, R., Choi, S., An, J. J., Simon, A. H., Angyal, M. S., Clevenger, L., Maniscalco, J., Nogami, T., Penny, C., and Kim, B. Y., J. Appl. Phys. 111, 093722 (2012).

Hu, C.-K., Rosenberg, R., and Lee, K. Y., Appl. Phys. Lett. 74, 2945 (1999).

Huang, T., Yang, T., Ke, J., Li, C., and Kao, C., J. Alloys Compd. 555, 237 (2013).

Hudlet, S., Guthmann, C., and Berger, J., J. Appl. Phys. 86, 5245 (1999).

Huisman, E. H., Trouwborst, M. L., Bakker, F. L., van Wees, B. J., and van der Molen, S. J., J. Appl. Phys. 109, 104305 (2011).

Hunley, D. P., Johnson, S. L., Flores, R. L., Sundararajan, A., and Strachan, D. R., J. Appl. Phys. 113, 234306 (2013).

Huntington, H. B. and Grone, A. R., J. Phys. Chem. Solids 20, 76 (1961).

Ichinokawa, T., Izumi, H., Haginoya, C., and Itoh, H., Phys. Rev. B 47, 9654 (1993). 
Ishida, H., Phys. Rev. B 49, 14610 (1994).

Ittah, N., Yutsis, I., and Selzer, Y., Nano Lett. 8, 3922 (2008).

Jelínek, P., Pérez, R., Ortega, J., and Flores, F., Nanotechnology 16, 1023 (2005).

Jelínek, P., Pérez, R., Ortega, J., and Flores, F., Phys. Rev. Lett. 96, 046803 (2006).

Jeong, W., Kim, K., Kim, Y., Lee, W., and Reddy, P., Sci. Rep. 4, 4975 (2014).

Johnston, D. E., Strachan, D. R., and Johnson, A. T. C., Nano Lett. 7, 2774 (2007).

Joo, Y.-C. and Thompson, C. V., J. Appl. Phys. 81, 6062 (1997).

Jung, Y., Vacic, A., Sun, Y., Hadjimichael, E., and Reed, M. A., Nanotechnology 23, 045301 (2012).

Kaeriyama, S., Sakamoto, T., Sunamura, H., Mizuno, M., Kawaura, H., Hasegawa, T., Terabe, K., Nakayama, T., and Aono, M., IEEE J. SolidState Circuits 40, 168 (2005).

Kaspers, M. R., Bernhart, A. M., Zu Heringdorf, F.-J. M., Dumpich, G., and Möller, R., J. Phys.: Condens. Matter 21, 265601 (2009).

Kastner, M. A., Rev. Mod. Phys. 64, 849 (1992).

Kayashima, S., Takahashi, K., Motoyama, M., and Shirakashi, J.-I., Jpn. J. Appl. Phys. 46, L907 (2007).

Kiessig, B., Schäfer, R., and Löhneysen, H. V., New J. Phys. 16, 013017 (2014).

Kiessig, B. A., "On electronic transport through single molecules in nanostructures," Ph.D. thesis (Karlsruhe Institute of Technology, 2012).

Kiguchi, M., Konishi, T., Miura, S., and Murakoshi, K., Nanotechnology 18, 424011 (2007).

Kim, K., Jeong, W. H., Lee, W. C., and Reddy, P., ACS Nano 6, 4248 (2012).

Kirchheim, R., Acta Metall. Mater. 40, 309 (1992).

Kirkendall, E., Thomassen, L., and Upthegrove, C., Trans. AIME 133, 186 (1939).

Kitamura, S.-I. and Iwatsuki, M., Jpn. J. Appl. Phys. 34, L145 (1995).

Kizuka, T. and Aoki, H., Appl. Phys. Express 2, 075003 (2009).

Kolesnychenko, O. Y., Shklyarevskii, O. I., and van Kempen, H., Rev. Sci. Instrum. 70, 1442 (1999).

Kondo, Y. and Takayanagi, K., Phys. Rev. Lett. 79, 3455 (1997).

Kondo, Y. and Takayanagi, K., Science 289, 606 (2000).

Korhonen, M. A., Borgesen, P., Tu, K. N., and Li, C.-Y., J. Appl. Phys. 73, 3790 (1993).

Korotkov, A. L., Bowman, M., McGuinness, H. J., and Davidovic, D., Nanotechnology 14, 42 (2003).

Kozlova, T., Rudneva, M., and Zandbergen, H. W., Nanotechnology 24, 505708 (2013).

Kraft, O. and Arzt, E., Appl. Phys. Lett. 66, 2063 (1995).

Kubatkin, S., Danilov, A., Hjort, M., Cornil, J., Bredas, J.-L., Stuhr-Hansen, N., Hedegard, P., and Bjørnholm, T., Nature 425, 698 (2003).

Kuhn, P., Krug, J., Hausser, F., and Voigt, A., Phys. Rev. Lett. 94, 166105 (2005).

Kulik, I. O., Sov. J. Low. Temp. Phys. 18, 302 (1992).

Kumar, P. and Sorbello, R. S., Thin Solid Films 25, 25 (1975).

Kunstmann, T., Schlarb, A., Fendrich, M., Wagner, T., Möller, R., and Hoffmann, R., Phys. Rev. B 71, 121403(R) (2005).

Kurui, Y., Oshima, Y., Okamoto, M., and Takayanagi, K., Phys. Rev. B 79, 165414 (2009).

Kurui, Y., Oshima, Y., and Takayanagi, K., J. Phys. Soc. Jpn. 76, 123601 (2007).

Labayen, M., Ramirez, C., Schattke, W., and Magnussen, O. M., Nat. Mater. 2, 783 (2003).

Lagos, M. J., Sato, F., Galvao, D. S., and Ugarte, D., Phys. Rev. Lett. 106, 055501 (2011)

Lambert, M. F., Goffman, M. F., Bourgoin, J. P., and Hesto, P., Nanotechnology 14, 772 (2003).

Landauer, R., Philos. Mag. 21, 863 (1970).

Landauer, R., Phys. Rev. B 14, 1474 (1976).

Landman, U. and Luedtke, W. D., J. Vac. Sci. Technol. B 9, 414 (1991).

Landman, U., Luedtke, W. D., Burnham, N. A., and Colton, R. J., Science 248, 454 (1990).

Lantz, M. A., Hug, H. J., Hoffmann, R., van Schendel, P. J. A., Kappenberger, P., Martin, S., Baratoff, A., and Güntherodt, H.-J., Science 291, 2580 (2001).

Latz, A., Sindermann, S. P., Brendel, L., Dumpich, G., zu Heringdorf, F.-J. M., and Wolf, D. E., J. Phys.: Condens. Matter 26, 055005 (2014).

Lee, W., Kim, K., Jeong, W., Zotti, L. A., Pauly, F., Cuevas, J. C., and Reddy, P., Nature 498, 209 (2013).
Lefebvre, J., Radosavljevic, M., and Johnson, A. T., Appl. Phys. Lett. 76, $3828(2000)$

Levine, L., Reiss, G., and Smith, D., Phys. Rev. B 48, 858 (1993).

Li, T., Hu, W., and Zhu, D., Adv. Mater. 22, 286 (2010).

Liang, W., Shores, M. P., Bockrath, M., Long, J. R., and Park, H., Nature 417, 725 (2002).

Lim, M. K., Chouliaras, V. A., Gan, C. L., and Dwyer, V. M., Microelectron. Reliab. 53, 1261 (2013).

Limot, L., Kröger, J., Berndt, R., Garcia-Lekue, A., and Hofer, W. A., Phys. Rev. Lett. 94, 126102 (2005).

Lin, R., Bammerlin, M., Hansen, O., Schlittler, R. R., and Bøggild, P., Nanotechnology 15, 1363 (2004).

Lloyd, J. R., J. Appl. Phys. 69, 7601 (1991).

Lloyd, J. R., Semicond. Sci. Technol. 12, 1177 (1997).

Lloyd, J. R., J. Phys. D: Appl. Phys. 32, R109 (1999).

Lodder, A., Europhys. Lett. 72, 774 (2005).

Lü, J.-T., Brandbyge, M., and Hedegård, P., Nano Lett. 10, 1657 (2010).

Lü, J.-T., Christensen, R. B., Wang, J.-S., Hedegård, P., and Brandbyge, M., Phys. Rev. Lett. 114, 096801 (2015).

Lucier, A. S., Mortensen, H., Sun, Y., and Grütter, P., Phys. Rev. B 72, 235420 (2005).

Luo, K. and Yao, Z., Appl. Phys. Lett. 95, 113115 (2009).

Lüthi, R., Schlittler, R. R., Brugger, J., Vettiger, P., Welland, M. E., and Gimzewski, J. K., Appl. Phys. Lett. 75, 1314 (1999).

Mahadevan, M. and Bradley, R. M., Phys. Rev. B 59, 11037 (1999).

Maldonado, A., Guillamon, I., Suderow, H., and Vieira, S., Rev. Sci. Instrum. 82, 073710 (2011)

Mamin, H. J., Guethner, P. H., and Rugar, D., Phys. Rev. Lett. 65, 2418 (1990).

Mares, A. I., Otte, A. F., Soukiassian, L. G., Smit, R. H. M., and van Ruitenbeek, J. M., Phys. Rev. B 70, 073401 (2004).

Mares, A. I. and van Ruitenbeek, J. M., Phys. Rev. B 72, 205402 (2005).

Martin, C. A., van Ruitenbeek, J. M., and van der Zant, H. S. J., Nanotechnology 21, 265201 (2010).

Martin, C. A., Smit, R. H. M., van der Zant, H. S. J., and van Ruitenbeek, J. M., Nano Lett. 9, 2940 (2009).

Masuda, H. and Kizuka, T., J. Nanosci. Nanotechnol. 14, 2436 (2014).

Maxwell, J. C., A Treatise of Electricity and Magnetism (Dover Publications, Inc., New York, 1891).

Mayer, T. M., Houston, J. E., Franklin, G. E., Erchak, A. A., and Michalske, T. A., J. Appl. Phys. 85, 8170 (1999).

Mehl, H., Biham, O., Millo, O., and Karimi, M., Phys. Rev. B 61, 4975 (2000).

Mehrez, H., Wlasenko, A., Larade, B., Taylor, J., Grütter, P., and Guo, H., Phys. Rev. B 65, 195419 (2002).

Meirav, U., Kastner, M. A., and Wind, S. J., Phys. Rev. Lett. 65, 771 (1990).

Mendez, J., Gomez-Herrero, J., Pascual, J. I., Saenz, J. J., Soler, J. M., and Bar, A. M., J. Vac. Sci. Technol. B 14, 1145 (1996).

Meyer, C., Klijn, J., Morgenstern, M., and Wiesendanger, R., Phys. Rev. Lett. 91, 076803 (2002).

Misbah, C., Pierre-Louis, O., and Saito, Y., Rev. Mod. Phys. 82, 981 (2010).

Mizobata, J.-I., Fujii, A., Kurokowa, S., and Sakai, A., Phys. Rev. B 68 , 155428 (2003).

Noncontact Atomic Force Microscopy, edited by Morita, S., Wiesendanger, R., and Meyer, E. (Springer-Verlag, Berlin, 2002).

Moth-Poulsen, K. and Bjørnholm, T., Nat. Nanotechnol. 4, 551 (2009).

Motto, P., Dimonte, A., Rattalino, I., Demarchi, D., Piccinini, G., and Civera, P., Nanoscale Res. Lett. 7, 113 (2012).

Muller, C. J., Krans, J. M., Todorov, T. N., and Reed, M. A., Phys. Rev. B 53, 1022 (1996).

Muller, C. J., van Ruitenbeek, J. M., and de Jongh, L. J., Phys. Rev. Lett. 69, 140 (1992).

Musket, R. G., McLean, W., Colmenares, C. A., Makowiecki, D. M., and Siekhaus, W. J., Appl. Surf. Sci. 10, 143 (1982).

Naitoh, Y., Horikawa, M., Abe, H., and Shimizu, T., Nanotechnology 17, 5669 (2006)

Natelson, D., Li, Y., and Herzog, J. B., Phys. Chem. Chem. Phys. 15, 5262 (2013).

Nielsen, S. K., Noat, Y., Brandbyge, M., Smit, R. H. M., Hansen, K., Chen, L. Y., Yanson, A. I., Besenbacher, F., and van Ruitenbeek, J. M., Phys. Rev. B 67, 245411 (2003).

Nikolic, B. and Allen, P. B., Phys. Rev. B 60, 3963 (1999). 
Nilius, N., Wallis, T. M., and Ho, W., Science 297, 1853 (2002).

Nilius, N., Wallis, T. M., and Ho, W., Phys. Rev. Lett. 90, 186102 (2003).

Noguchi, Y., Ueda, R., Kubota, T., Kamikado, T., Yokoyama, S., and Nagase, T., Thin Solid Films 516, 2762 (2008).

Oates, A. S., ECS J. Solid State Sci. Technol. 4, N3168 (2015).

Ohnishi, H., Kondo, Y., and Takayanagi, K., Nature 395, 780 (1998).

Okino, H., Matsuda, I., Hobara, R., Hosomura, Y., Hasegawa, S., and Bennett, P. A., Appl. Phys. Lett. 86, 233108 (2005).

Oliver, D. J., Maassen, J., Ouali, M. E., Paul, W., Hagedorn, T., Miyahara, Y., Qi, Y., Guo, H., and Grütter, P., PNAS 109, 19097 (2012).

O’Neill, K., Osorio, E. A., and van der Zant, H. S. J., Appl. Phys. Lett. 90, 133109 (2007).

Oon, C. H. and Thong, J. T. L., Nanotechnology 15, 687 (2004).

de Orio, R. L., Ceric, H., and Selberherr, S., Microelectron. Reliab. 50, 775 (2010).

Oshima, Y. and Kurui, Y., Phys. Rev. B 87, 081404(R) (2013).

Oshima, Y., Kurui, Y., Nguyen, H. D., Ono, T., and Takayanagi, K., Phys. Rev. B 84, 035401 (2011).

Osorio, E. A., Bjørnholm, T., Lehn, J.-M., Ruben, M., and van der Zant, H. S. J., J. Phys.: Condens. Matter 20, 374121 (2008).

Otsuka, Y., Naitoh, Y., Matsumoto, T., Mizutani, W., Tabata, H., and Kawai, T., Nanotechnology 15, 1639 (2004).

Park, H., Lim, A. K. L., Alivisatos, A. P., Park, J., and McEuen, P. L., Appl. Phys. Lett. 75, 301 (1999).

Park, J., Pasupathy, A. N., Goldsmith, J. I., Chang, C., Yaish, Y., Petta, J. R., Rinkoski, M., Sethna, J. P., Abruna, H. D., McEuen, P. L., and Ralph, D. C., Nature 417, 722 (2002).

Paul, W., Oliver, D., and Grütter, P., Phys. Chem. Chem. Phys. 16, 8201 (2014).

Paul, W., Oliver, D., Miyahara, Y., and Grütter, P., Nanotechnology 24, 475704 (2013)

Pauly, F., Viljas, J. K., Bürkle, M., Dreher, M., Nielaba, P., and Cuevas, J. C., Phys. Rev. B 84, 195420 (2011).

Pecchia, A. and Carlo, A. D., Rep. Prog. Phys. 67, 1497 (2004).

Pérez León, C., Drees, H., Wippermann, S. M., Marz, M., and HoffmannVogel, R., J. Phys. Chem. Lett. 7, 426 (2016).

Perrin, M. L., Burzurí, E., and van der Zant, H. S. J., Chem. Soc. Rev. 44, 902 (2015).

Pierce, D. G. and Brusius, P. G., Microelectron. Reliab. 37, 1053 (1997).

Pierre-Louis, O. and Einstein, T. L., Phys. Rev. B 62, 13697 (2000).

Puebla-Hellmann, G. and Wallraff, A., Appl. Phys. Lett. 101, 053108 (2012).

Ralls, K. S., Buhrman, R. A., and Tiberio, R. C., Appl. Phys. Lett. 55, 2459 (1989).

Ralls, K. S., Ralph, D. C., and Buhrman, R. A., Phys. Rev. B 40, 11561 (1989).

Ralph, D. C. and Buhrman, R. A., Phys. Rev. Lett. 72, 3401 (1994).

Regan, B. C., Aloni, S., Ritchie, R. O., Dahmen, U., and Zettl, A., Nature 428, 924 (2004).

Reiss, G., Levine, L. E., and Smith, D. A., J. Vac. Sci. Technol. B 11, 108 (1992).

Roberts, C. J., Hoffmann-Millack, B., and Steer, W. S., J. Vac. Sci. Technol. B 9, 841 (1991)

Rodrigues, V., Fuhrer, T., and Ugarte, D., Phys. Rev. Lett. 85, 4124 (2000).

Rosei, F., J. Phys.: Condens. Matter 16, S1373 (2004).

Rous, P. J. and Bly, D. N., Phys. Rev. B 62, 8478 (2000).

Rous, P. J., Yongsunthon, R., Stanishevsky, A., and Williams, E. D., J. Appl. Phys. 95, 2477 (2004).

Rudneva, M., Gao, B., Prins, F., Xu, Q., van der Zant, H. S., and Zandbergen, H. W., Microsc. Microanal. 19, 43 (2013).

Rudneva, M., Kozlova, T., and Zandbergen, H. W., Ultramicroscopy 134. 155 (2013).

van Ruitenbeek, J. M., Alvarez, A., Pineyro, I., Grahmann, C., Joyez, P., Devoret, M. H., Esteve, D., and Urbina, C., Rev. Sci. Instrum. 67, 108 (1996).

Sabater, C., Untiedt, C., and van Ruitenbeek, J. M., Beilstein J. Nanotechnol. 6, 2338 (2015).

Samitsu, S., Shimomura, T., Ito, K., Fujimori, M., Heike, S., and Hashizume, T., Appl. Phys. Lett. 86, 233103 (2005).

Sanchez, J. E., Kraft, O., and Arzt, E., Appl. Phys. Lett. 61, 3121 (1992).

Sangiao, S., Michalik, J. M., Casado, L., Martinez-Velarte, M. C., Morellon, L., Ibarra, M. R., and Teresa, J. M. D., Phys. Chem. Chem. Phys. 15, 5132 (2013).
Sattar, A., Fostner, S., and Brown, S. A., Phys. Rev. Lett. 111, 136808 (2013).

Savu, V., van den Boogaart, M. A., Brugger, J., Arcamone, J., Sansa, M., and Perez-Murano, F., J. Vac. Sci. Technol. B 26, 2054 (2008).

Scheer, E., Agraï, N., Cuevas, J. C., Levy Yeyati, A., Ludoph, B., MartinRodero, A., Rubio Bollinger, G., van Ruitenbeek, J. M., and Urbina, C., Nature 394, 154 (1998).

Schintke, S. and Schneider, W. D., J. Phys.-Condens. Matter 16, R49 (2004).

Schirm, C., Matt, M., Pauly, F., Cuevas, J. C., Nielaba, P., and Scheer, E., Nat. Nanotechnol. 8, 645 (2013).

Schneider, W.-D., Surf. Sci. 514, 74 (2002).

Schönenberger, C., van Houten, H., and Donkersloot, H. C., Europhys. Lett. 20, 249 (1992).

Sellers, M. S., Schultz, A. J., Basaran, C., and Kofke, D. A., J. Appl. Phys. 110, 013528 (2011).

Sen, F. G. and Aydinol, M. K., J. Appl. Phys. 104, 073510 (2008).

Sharvin, Y. V., Sov. Phys. JETP 21, 655 (1965).

Shatzkes, M. and Lloyd, J. R., J. Appl. Phys. 59, 3890 (1986).

Shih, V. C.-Y., Zheng, S., Chang, A., and Tai, Y.-C., in 12th International Conference on Solid-State Sensors, Actuators and Microsystems. Digest of Technical Papers, TRANSDUCERS'03 (2003), Vol. 2, p. 1530.

Shingubara, S., Nakasaki, Y., and Kaneko, H., Appl. Phys. Lett. 58, 42 (1991).

Simmons, J. G., J. Appl. Phys. 34, 1793 (1963).

Sindermann, S., Witt, C., Spoddig, D., von Hoegen, M. H., Dumpich, G., and Zu Heringdorf, F.-J. M., Rev. Sci. Instrum. 82, 123907 (2011).

Sindermann, S. P., Latz, A., Dumpich, G., Wolf, D. E., and Zu Heringdorf, F.-J. M., J. Appl. Phys. 113, 134505 (2013).

Smit, R. H. M., Noat, Y., Untiedt, C., Lang, N. D., van Hemert, M. C., and van Ruitenbeek, J. M., Nature 419, 906 (2002).

Smit, R. H. M., Untiedt, C., and van Ruitenbeek, J. M., Nanotechnology 15, S472 (2004).

Smith, D. P. E., Science 269, 371 (1995).

Solenov, D. and Velizhanin, K. A., Phys. Rev. Lett. 109, 095504 (2012).

Sondheimer, E. H., Adv. Phys. 1, 1 (1952).

Song, H., Reed, M. A., and Lee, T., Adv. Mater. 23, 1583 (2011).

Sorbello, R., Phys. Rev. B 31, 798 (1985).

Sorbello, R. S., Solid State Phys. 51, 159 (1998).

Sordan, R., Balasubramanian, K., Burghard, M., and Kern, K., Appl. Phys. Lett. 87, 013106 (2005).

Stahlmecke, B. and Dumpich, G., Appl. Phys. Lett. 90, 043517 (2007a).

Stahlmecke, B. and Dumpich, G., J. Phys.: Condens. Matter 19, 046210 (2007b).

Stahlmecke, B., Zu Heringdorf, F.-J. M., Chelaru, L. I., von Hoegen, M. H., and Dumpich, G., Appl. Phys. Lett. 88, 053122 (2006).

Steinmann, P. and Weaver, J. M. R., Appl. Phys. Lett. 86, 063104 (2005).

Stepanyuk, V. S., Bruno, P., Klavsyuk, A. L., Baranov, A. N., Hergert, W., Saletsky, A. M., and Mertig, I., Phys. Rev. B 69, 033302 (2004).

Stierle, A., Carstanjen, H.-D., Hofmann, S., and Seeck, O. H., in Nanoelectronics and Information Technology, edited by R. Waser (WileyVCH, Weinheim, Berlin, 2012).

Stöffler, D., Fostner, S., Grütter, P., and Hoffmann-Vogel, R., Phys. Rev. B 85, 033404 (2012).

Stöffler, D. and Hoffmann-Vogel, R., J. Vac. Sci. Technol. B 33, 013201 (2015).

Stöffler, D., Löhneysen, H. V., and Hoffmann-Vogel, R., Nanoscale 3, 3391 (2011).

Stöffler, D., Marz, M., Kießig, B., Tomanic, T., Schäfer, R., Löhneysen, H. v., and Hoffmann-Vogel, R., Phys. Rev. B 90, 115406 (2014).

Strachan, D. R., Johnston, D. E., Guiton, B. S., Datta, S. S., Davies, P. K., Bonnell, D. A., and Johnson, A. T. C., Phys. Rev. Lett. 100, 056805 (2008).

Strachan, D. R., Smith, D. E., Fischbein, M. D., Johnston, D. E., Guiton, B. S., Drndic, M., Bonnell, D. A., and Johnson, A. T., Nano Lett. 6, 441 (2006).

Strachan, D. R., Smith, D. E., Johnston, D. E., Park, T.-H., Therien, M. J., Bonnell, D. A., and Johnson, A. T., Appl. Phys. Lett. 86, 043109 (2005).

Strigl, F., Espy, C., Bückle, M., Scheer, E., and Pietsch, T., Nat. Commun. 6, $6172(2015)$.

Stroscio, J. A. and Eigler, D. M., Science 254, 1319 (1991).

Stroscio, J. A., Whitman, L. J., Dragoset, R. A., and Celotta, R. J., Nanotechnology 3, 133 (1992). 
Suga, H., Sumiya, T., Horikawa, M., Kumaragurubaran, S., Shimizu, T., and Naitoh, Y., Jpn. J. Appl. Phys. 50, 06GF11 (2011).

Suga, H., Suzuki, H., Shinomura, Y., Kashiwabara, S., Tsukagoshi, K., Shimizu, T., and Naitoh, Y., Sci. Rep. 6, 34961 (2016).

Sugimoto, Y., Abe, M., Hirayama, S., Oyabu, N., Custance, O., and Morita, S., Nat. Mater. 4, 156 (2005).

Sukharev, V., Zschech, E., and Nix, W. D., J. Appl. Phys. 102, 053505 (2007).

Takahashi, Y., Nishino, Y., Furukawa, H., Kubo, H., Yamauchi, K., Ishikawa, T., and Matsubara, E., J. Appl. Phys. 105, 124911 (2009).

Tanielian, M. H., Greegor, R. B., Nielsen, J. A., and Parazzoli, C. G., Appl. Phys. Lett. 99, 123104 (2011).

Tao, C., Cullen, W. G., and Williams, E. D., Science 328, 736 (2010).

Tavazza, F., Smith, D. T., Levine, L. E., Pratt, J. R., and Chaka, A. M., Phys. Rev. Lett. 107, 126802 (2011).

Taychatanapat, T., Bolotin, K. I., Kuemmeth, F., and Ralph, D. C., Nano Lett. 7, 652 (2007)

Terabe, K., Hasegawa, T., Nakayama, T., and Aono, M., Nature 433, 47 (2005).

Ternes, M., Gonzalez, C., Lutz, C. P., Hapala, P., Giessibl, F. J., Jelinek, P., and Heinrich, A. J., Phys. Rev. Lett. 106, 016802 (2011).

Ternes, M., Lutz, C. P., Hirjibehedin, C. F., Giessibl, F. J., and Heinrich, A. J., Science 319, 1066 (2008).

Thijssen, W. H. A., Marjenburgh, D., Bremmer, R. H., and van Ruitenbeek, J. M., Phys. Rev. Lett. 96, 026806 (2006).

Todorov, T. N., Philos. Mag. B 77, 965 (1998).

Todorov, T. N., J. Phys.: Condens. Matter 14, 3049 (2002).

Todorov, T. N., Dundas, D., Lü, J.-T., Brandbyge, M., and Hedegård, P., Eur. J. Phys. 35, 065004 (2014).

Todorov, T. N., Hoekstra, J., and Sutton, A., Phys. Rev. Lett. 86, 3606 (2001).

Tohmyoh, H., Appl. Phys. Lett. 102, 084107 (2013).

Topinka, M. A., LeRoy, B. J., Shaw, S. E. J., Heller, E. J., Westervelt, R. M., Maranowski, K. D., and Gossard, A. C., Science 289, 2323 (2000).

Torres, J. A., Pascual, J. I., and Saenz, J. J., Phys. Rev. B 94, 16581 (1994).

Tromp, R. M., J. Phys.: Condens. Matter 1, 10211 (1989).

Trouwborst, M. L., Huisman, E. H., Bakker, F. L., van der Molen, S. J., and van Wees, B. J., Phys. Rev. Lett. 100, 175502 (2008).

Trouwborst, M. L., Martin, C. A., Smit, R. H. M., Guedon, C. M., Baart, T. A., van der Molen, S. J., and van Ruitenbeek, J. M., Nano Lett. 11, 614 (2011).

Trouwborst, M. L., van der Molen, S. J., and van Wees, B. J., J. Appl. Phys. 99, 114316 (2006).

Tseng, A. A., Notargiacomo, A., and Chen, T. P., J. Vac. Sci. Technol. B 23, 877 (2005).

Tsong, T. T., Phys. Rev. B 44, 13703 (1991).

Tsutsui, M., Kawai, T., and Taniguchi, M., Sci. Rep. 2, 217 (2012)

Tsutsui, M., Kurokawa, S., and Sakai, A., Nanotechnology 17, 5334 (2006).

Tsutsui, M., Shoji, K., Taniguchi, M., and Kawai, T., Nano Lett. 8, 345 (2008).

Untiedt, C., Dekker, D. M. T., Djukic, D., and van Ruitenbeek, J. M., Phys. Rev. B 69, 081401R (2004).

Vazquez-Mena, O., Gross, L., Xie, S., Villanueva, L. G., and Brugger, J., Microelectron. Eng. 132, 236 (2015).

Venkataraman, L., Klare, J. E., Nuckolls, C., Hybertsen, M. S., and Steigerwald, M. L., Nature 442, 904 (2006).

Wang, N., Bevan, K. H., and Provatas, N., Phys. Rev. Lett. 117, 155901 (2016).

Ward, D. R., Corley, D. A., Tour, J. M., and Natelson, D., Nat. Nanotechnol. 6, 33 (2011).
Ward, D. R., Halas, N. J., and Natelson, D., Appl. Phys. Lett. 93, 213108 (2008).

van Wees, B. J., van Houten, H., Beenakker, C. W. J., Williamson, J. G., Kouwenhoven, L. P., van der Marel, D., and Foxon, C. T., Phys. Rev. Lett. 60, 848 (1988).

Weiss, C., Wagner, C., Kleimann, C., Rohlfing, M., Tautz, F. S., and Temirov, R., Phys. Rev. Lett. 105, 086103 (2010).

Wexler, G., Proc. Phys. Soc. 89, 927 (1966).

Weymouth, A. J., Wutscher, T., Welker, J., Hofmann, T., and Giessibl, F. J., Phys. Rev. Lett. 106, 226801 (2011).

Wharam, D. A., Thornton, T. J., Newbury, R., Pepper, M., Ahmed, H., Frost, J. E. F., Hasko, D. G., Peacock, D. C., Ritchie, D. A., and Jones, G. A. C., J. Phys. C: Solid State 21, L209 (1988).

Wheeler, P. J., Chen, R., and Natelson, D., Phys. Rev. B 87, 155411 (2013).

Wolf, C. R., Thonke, K., and Sauer, R., Appl. Phys. Lett. 96, 142108 (2010).

Wouters, D. and Schubert, U. S., Angew. Chem. Int. Ed. 43, 2480 (2004).

Wu, Z.-J. and Ho, P. S., Appl. Phys. Lett. 101, 101601 (2012).

Wu, Z. M., Steinacher, M., Huber, R., Calame, M., van der Molen, S. J., and Schönenberger, C., Appl. Phys. Lett. 91, 053118 (2007).

Xiang, A., Hou, S., and Liao, J., Appl. Phys. Lett. 104, 223113 (2014).

Xie, F.-Q., Nittler, L., Obermair, C., and Schimmel, T., Phys. Rev. Lett. 93, 128303 (2004).

Yagi, M., Saito, T., and Shirakashi, J.-I., J. Vac. Sci. Technol. B 33, 051806 (2015).

Yang, Z., Chshiev, M., Zwolak, M., Chen, Y.-C., and Ventra, M. D., Phys. Rev. B 71, 041402(R) (2005).

Yanson, A. I. and van Ruitenbeek, J. M., Phys. Rev. Lett. 79, 2157 (1997).

Yanson, A. I., Yanson, I. K., and van Ruitenbeek, J. M., Nature 400, 144 (1999).

Yanson, A. I., Yanson, I. K., and Ruitenbeek, J. M. V., Phys. Rev. Lett. 84, $5832(2000)$

Yanson, A. I., Yanson, I. K., and Ruitenbeek, J. M. V., Phys. Rev. Lett. 87, 216805 (2001).

Yanson, I. K., Shklyarevskii, O. I., Csonka, S., Kempen, H. V., Speller, S., Yanson, A. I., and Ruitenbeek, J. M. V., Phys. Rev. Lett. 95, 256806 (2005).

Yokogawa, S. and Tsuchiya, H., J. Appl. Phys. 101, 013513 (2007).

Yoshida, K., Oshima, Y., and Takayanagi, K., Appl. Phys. Lett. 87, 103104 (2005).

Yoshida, K., Umeno, A., Sakata, S., and Hirakawa, K., Jpn. J. Appl. Phys. 48, 120216 (2009).

Yoshida, K., Umeno, A., Sakata, S., and Hirakawa, K., Appl. Phys. Express 3, 045001 (2010).

Young, R., Ward, J., and Scire, F., Phys. Rev. Lett. 27, 922 (1971).

Zahl, P., Bammerlin, M., Meyer, G., and Schlittler, R. R., Rev. Sci. Instrum. 76, 023707 (2005)

van der Zant, H. S. J., Osorio, E. A., Poot, M., and O’Neill, K., Phys. Status Solidi B 243, 3408 (2006).

Zhang, C.-H., Bürki, J., and Stafford, C. A., Phys. Rev. B 71, 235404 (2005).

Zhang, R., Rungger, I., Sanvito, S., and Hou, S., Phys. Rev. B 84, 085445 (2011).

Zhang, Y., Wahl, P., and Kern, K., Nano Lett. 11, 3838 (2011).

Zhao, J., Garay, J. E., Anselmi-Tamburini, U., and Munir, Z. A., J. Appl. Phys. 102, 114902 (2007).

Zhao, J. and Zhu, J., J. Appl. Phys. 107, 084308 (2010).

Zschech, E., Huebner, R., Chumakov, D., Aubel, O., Friedrich, D., Guttmann, P., Heim, S., and Schneider, G., J. Appl. Phys. 106, 093711 (2009). 